\title{
ESTABILIZACIÓN DE SISTEMAS AFINES MEDIANTE CONTROLES ACOTADOS
}

\author{
TESIS QUE PRESENTA EL \\ M. EN C. JULIO ERNESTO SOLÍS DAUN \\ PARA LA OBTENCIÓN DEL GRADO DF, \\ DOCTOR EN CIENCIAS
}

ASESOR DE LA TESIS

DR. RODOLFO SUÁREZ CORTES

DIVISIÓN DE CIENCIAS BÁSICAS E INGENIERÍA UNIVERSIDAD AUTÓNOMA METROPOLITANA-IZTAPALAPA 


\section{Agradecimientos}

A mis padres:

José G. Solís Miranda (Q.P.D.)

$y$

Julieta Daun de Solís

por su amor y apoyo.

A mis hermanos:

Landy, Linda, Orlando y Fernando, por su cariño

y aliento. 
A mis maestros, con respecto y gratitud.

A los profesores sinodales:

Dr. Rodolfo Suárez Cortes,

Dr. José de Jesús Álvarez Ramírez,

Dr. Ernesto Lacomba Zamora,

Dr. Carlos Ibarra Valdez,

Dr. Guillermo Fernández Anaya,

Dr. Eduardo Aranda Bricaire,

por su consideración con este trabajo.

Muy en especial, expreso mi agradecimicnto a mi asesor, Dr. Rodolfo Suárez, por su motivación para el buen término de la presente obra; al Dr. José Álvarez, por sus valiosas sugerencias y entusiasmo; y al Dr. Carlos Ibarra por su interés y tiempo.

También, aunque no por eso en último lugar, manifiesto mi sincero aprecio a mis todos amigos y compañeros de trabajo.

Finalmente, deseo hacer patente mi agradecimiento al Consejo Nacional de Ciencia y Tecnología (CONACyT), por el apoyo ofrecido para el desarrollo de este trabajo. 


\section{Índice}

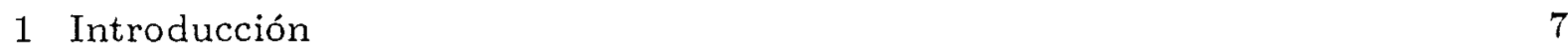

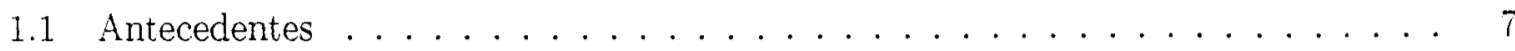

1.2 Descripción de las contribuciones principales . . . . . . . . . . . . 9

2 Estabilización de Sistemas Lineales Controlables con Controles Subóptimos 13

2.1 Introducción . . . . . . . . . . . . . . . . . . 13

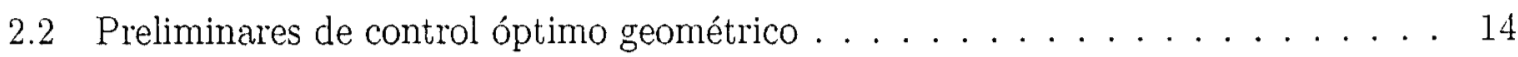

2.3 Estabilizador asintótico continuo y acotado . . . . . . . . . . . . . 16

2.4 Estabilización global asintótica del $n$-integrador . . . . . . . . . . . . . . 19

2.5 Sistemas en el plano . . . . . . . . . . . . . . . . . . 22

3 Sistemas Lineales con Entradas Acotadas: Estabilización Global con Asignación de Eigenvalores

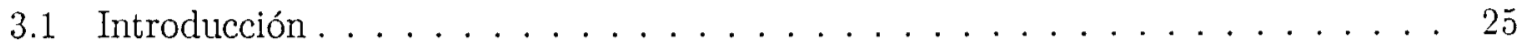

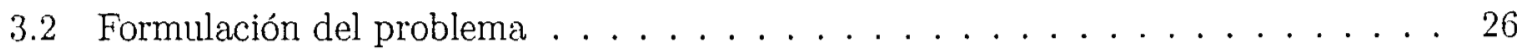

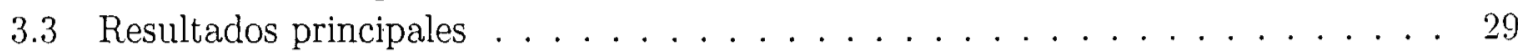

3.4 Ejemplo numérico . . . . . . . . . . . . . . . . . 32

4 Estabilización Global y Semiglobal de Sistemas Lineales Mediante Controles Acotados $\quad 35$

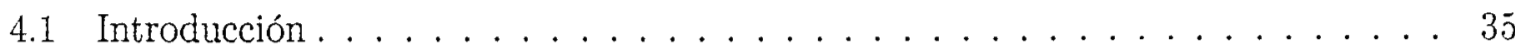

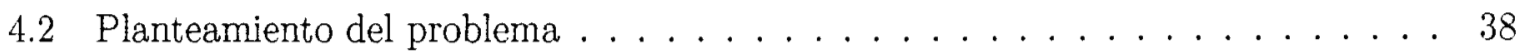

4.2 .1 Ideas geométricas preliminares . . . . . . . . . . . . . . 40

4.3 Un problema de programación paramétrica . . . . . . . . . . . . . . . . . . . . . . . . . . .

4.4 Resultado de estabilización global . . . . . . . . . . . . . . . . . 43

4.5 Estabilización semiglobal para los sistemas lineales ACOCA . . . . . . . . . . . 47

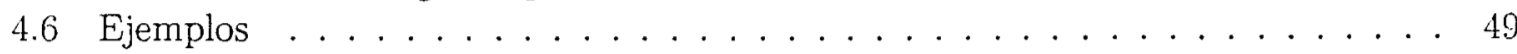

5 Estabilización de Sistemas No Lineales Mediante Controles Acotados: Enfoque de Programación Paramétrica $\quad \mathbf{5 5}$

5.1 Introducción . . . . . . . . . . . . . . . . . 55

5.2 Preliminares y planteamiento del problema . . . . . . . . . . . . . 58

5.2 .1 Ideas geométricas preliminares . . . . . . . . . . . . . . . . . . . . . . . . . . . . 60

5.3 Resultados generales . . . . . . . . . . . . . . . . . . . 61 . . . . . . . . . 61

5.4 Método de multiplicadores de Lagrange . . . . . . . . . . . . . . . 66 
5.4 .1 El caso de entrada escalar . . . . . . . . . . . . . . . . 67

5.5 Una clase de sistemas homogéneos . . . . . . . . . . . . . . . 68

5.5.1 Sistemas estabilizables global asintóticamente mediante control lineal . . . 71

5.5.2 Sistemas bilineales homogéneos: $f(x)=A x$ y $g_{j}(x)=D_{j} x, j=1, \ldots, m$. 74

5.6 Sistemas bilineales no homogéneos: $f(x)=A x$ y $g(x)=D x+b \ldots \ldots$. . . . 78

6 Conclusiones $\quad 85$

7 Apéndice $\quad 89$ 


\section{Lista de Acrónimos}

ACOCA : Asintóticamente Controlable al Origen con Controles Acotados.

DEC : Detectable En Cero.

EAR : Ecuación Algebraica de Riccati.

EDR : Ecuación Diferencial de Riccati.

EGA : Estabilización Global Asintótica.

J-Q : Jurdjevic-Quinn.

LQ : Linear-Quadratic.

PRNP : Parte Real No Positiva.

RCA : Retroalimentación de Control Acotada.

RLS : Retroalimentación Lineal Saturada.

RS : Retroalimentación Saturada. 


\section{INTRODUCCIÓN}

\subsection{Antecedentes}

El estudio realizado en esta Tesis se sitúa dentro de la denominada Teoría de Control. Dentro de la problemática general entre la controlabilidad y la estabilización de los sistemas de control, nos hemos centrado en el problema de considerar restricciones sobre los controles. Una característica natural de todas las aplicaciones radica en que el diseño de controles contemplen restricciones (e.g., cotas sobre sus magnitudes e incluso en sus derivadas). Este problema nos condujo al estudio de aspectos particularmente interesantes y novedosos, mismos que dieron lugar a nuevas técnicas para afrontar este problema.

En esta Tesis estudiaremos el problema de la estabilización global asintótica (EGA) de los sistemas afines, y con particular énfasis de los sistemas lineales, mediante retroalimentaciones de control acotadas (RCA), $u=u(x)$, sujetas a restricciones sobre sus magnitudes y también sobre sus derivadas.

Con respecto a los sistemas lineales, en años recientes ha surgido un renovado interés respecto al problema de la estabilización global mediante las RCA. Con el objeto de estudiar el problema anterior, se llegó a la caracterización de cierta clase de sistemas lineales: Sistema lineal asintóticamente controlable al origen con controles acotados (ACOCA). Es bien sabido, que un sistema lineal es ACOCA sii es estabilizable mediante controles arbitrariamente pequeños, o de manera equivalente (criterio algebraico), sii el sistema es estabilizable (i.e., todos sus modos no controlables tienen parte real negativa) y su dinámica libre asociada tiene todos sus eigenvalores con parte real no positiva (PRNP), cf. por ejemplo, Lee-Markus [32], Schmitendorf-Barmish [49] y Sontag [56]. Además, el enfoque natural de considerar saturaciones de retroalimentaciones lineales tiene el inconveniente de que la estabilización global asintótica no puede garantizarse a priori. En específico, Fuller [12] mostró que para el denominado $n$-integrador $(n \geq 3)$, una saturación hard no conduce a la estabilización global. A pesar de este resultado negativo, SontagSussmann [57) probaron que la EGA de los sistema lineales ACOCA puede realizarse mediante la clase general de las funciones RCA. Sin embargo, el diseño de controles en ese trabajo estaba basado en un procedimiento recursivo muy complicado (computacionalmente difícil). Teel [65] introdujo una metodología que empleaba combinaciones lineales y composiciones anidadas de saturaciones de retroalimentaciones lineales (enfoque de saturaciones anidadas) para obtener la EGA del n-integrador. Finalmente, Sussmann-Sontag-Yang [64] generalizaron ese método para afrontar el problema anterior, en vista de lo cual obtuvieron la EGA de los sistemas lineales ACOCA, suponiendo además, funciones de saturación más generales.

Una antigua solución al problema anterior, i.e., llevar cualquier estado inicial al origen, en tiempo finito, bajo el supuesto de considerar controles acotados (usualmente discontinuos) fue obtenida con la teoría de control de tiempo óptimo [32]. Empero, la derivación de funciones de control retroalimentado en tiempo óptimo puede ser muy engorrosa y su subsecuente implementación es muy difícil: se requiere de enormes cantidades de memoria on-line para almacenar 
hipersuperficies de cambio (switching) muy intricadas. En otros términos, su construcción necesita del conocimiento de los conjuntos controlables de los sistemas lineales. Este último aspecto plantea un problema sumamente difícil (en general, abierto). En particular, Korobov-Sklyar, obtuvieron soluciones analíticas al problema de tiempo óptimo para el n-integrador [29], y para sistemas con control escalar cuyas dinámicas asociadas tienen eigenvalores puramente imaginarios [30]. Hasta ahora, el papel de la teoría de control óptimo se reduce más bien a producir patrones a considerar como metas en sistemas reales: una ayuda para construir sistemas de control subóptimos. Sobre esta base, e interesado en hallar una solución suave al problema de control en tiempo subóptimo, Komarov [27] usó una estimación interna elipsoidal de los conjuntos controlables de sistemas lineales no autónomos. Un parámetro (o más bien una matriz parametrizada) de tal aproximación elipsoidal se obtiene resolviendo una ecuación diferencial matricial no lineal. El estabilizador resultante es una función de control acotada y subóptima de tiempo finito, la cual es suave, excepto en el origen. Sin embargo, la región de atracción no está bien determinada, al igual que falta establecer las condiciones para la existencia de soluciones para esa ecuación diferencial matricial en el origen (una singularidad en este caso). Usando un enfoque alternativo, pero no involucrado en obtener una solución subóptima, Korobov [28] se abocó al problema de la estabilización en tiempo finito del $n$-integrator, mediante las denominadas funciones de controlabilidad (funciones tipo Lyapunov). Ese método fue extendido por Gavrilyako-Korobov-Sklyar [15] a fin de obtener la estabilidad global en tiempo finito para los sistemas lineales controlables PRNP. Para ello, debe resolverse una ecuación integral no lineal. Empero, el problema de hallar sus soluciones suele tornarse bastante difícil.

Por contraste con el caso de los sistemas lineales, la caracterización de sistemas no lineales que sean del tipo ACOCA permanece aun como un problema abierto. Además, las estrategias generales que se han propuesto para estabilizar los sistemas no lineales mediante controles acotados son relativamente pocas, siendo principalmente enfocadas a los sistemas bilineales.

Entre las herramientas que usualmente se emplean para la estabilización de sistemas no lineales se hallan el análisis de Lyapunov, el principio de invariancia de LaSalle, la teoría de la variedad central, y álgebras de Lie [5]-[19]-[23]-[31]-[35]-[33]. A este respecto, Jurdjevic-Quim (J-Q) [23] desarrollaron un enfoque exitoso, basado en una condición del tipo de rango de controlabilidad definida en términos de álgebras de Lie: la llamada condición ad. En [23], se consideró el caso sistemas afines con entrada escalar cuyas dinámicas libres son lineales y la matriz de transición de estados es unitaria. Para sistemas bilineales con entrada escalar, los siguientes resultados fueron obtenidos bajo la misma línea de la técnica de J-Q: (i) Slemrod [50] propuso una mejora a la condición ad para obtener un control saturado que estabiliza global asintóticamente la clase de sistemas bilineales homogéneos cuya matriz de transición de estados es antisimétrica; y (ii) Gauthier y Kupka [14| investigaron el problema de la EGA de los sistemas bilineales con dinámica libre disipativa, mediante leyes de retroalimentación suaves y controles discontinuos. Estos últimos autores también presentaron condiciones para obtener la EGA de sistemas no lineales mediante controles retroalimentados suaves arbitrariamente pequeños -una propiedad que caracteriza la clase de los sistemas lineales ACOCA.

Recientemente, sobre la línea de investigación de [14], Lin [34] obtuvo la EGA de cierta clase de sistemas afines con entradas múltiples, cuya dinámica libre es lineal y Lyapunov estable, bajo la consideración tanto de funciones retroalimentadas suaves (no acotadas) o acotadas ("estabilizadores pequeños" discontinuos). Lin derivó una condición ad, que generaliza los resultados obtenidos por Lee-Arapostathis [31] y Byrnes-Isidori-Willems [5], y por ende, mejora 
la de J-Q. En Lin [35], el método previo fue extendido para obtener la EGA para la clase general de sistemas no afines pasivos. En virtud de que los sistemas pasivos tienen la propiedad tipo ACOCA [35] antes mencionada, éstos pueden pensarse como sistemas no lineales "casi lineales".

Es importante remarcar que en los métodos antes mencionados, los controles acotados son bien sea (i) del tipo de baja ganancia -que implica que la estabilización global asintótica se obtiene a expensas de variaciones lentas de la función de control, y por lo tanto obteniendo (probablemente) un pobre desempeño y largos tiempos para terminar (settling times), o bien (ii) controles bang-bang -que pueden concebirse como controles con ganancias infinitas, lo cual impide sus usos en algunas aplicaciones.

En la siguiente sección presentamos un panorama de las contribuciones de nuestro trabajo de investigación para abordar la problemática anterior. En primera instancia, mencionamos los resultados que obtuvimos para los sistemas lineales. Nuestro primer enfoque (Capítulo 2 o [62]) está basado en el método propuesto por Komarov [27], mediante el cual se obtiene una RCA de tipo global para el $n$-integrador, y se establece una relación entre la estabilización asintótica y la de tiempo finito. Mediante un segundo método (Capítulo 3 o [60]), obtenemos una extensión del (conocido) resultado de asignación de valores propios para sistemas lineales, bajo la restricción de controles acotados. Finalmente (Capítulo 4 o [54] y Capítulo 5 o [55]), exponemos una metodología que permite extender resultados de estabilización con control acotado para el caso de los sistemas afines que a lazo abierto son Lyapunov estables. Con este enfoque, se obtiene un control acotado (mediante cierta función-peso) que estabiliza globalmente el sistema. La construcción de tal función-peso depende de la solución de un problema de optimización paramétrica. Además, se proporcionan condiciones para que los controles tengan derivadas globalmente acotadas.

\subsection{Descripción de las contribuciones principales}

Dentro del marco de la teoría clásica para la estabilización de sistemas lineales, los controles no acotados pueden construirse usando funciones de Lyapunov de tipo cuadrático. Sin embargo, la imposición de cotas conlleva a considerar funciones de Lyapunov más generales. En nuestro trabajo se definen tales funciones mediante parametrizaciones apropiadas de funciones de Lyapunov cuadráticas, lo cual se traduce en considerar funciones definidas en forma implícita. Con esto, esta metodología permite el diseño de controles acotados, retroalimentados y "suaves", aunque definidos implícitamente, es decir, el sistema a lazo cerrado, resultado de aplicar el control implícito, consta de un sistema de ecuaciones diferenciales más una ecuación algebraica no lineal (para el cálculo del control). En concreto, presentamos dos técnicas para llevar a efecto tales parametrizaciones, que describimos en los siguientes dos párrafos.

Con base en el enfoque de Komarov [27], y especializados a sistemas lineales autónomos controlables de una entrada, en el Capítulo 2 (o [62]), establecemos una conexión entre la estabilización asintótica y la de tiempo finito vía un rediseñamiento del controlador. Mediante aproximaciones internas (las funciones de Lyapunov implícitas) al conjunto controlable en su evolución con el tiempo de un sistema lineal, se construye un control retroalimentado, "suave" 
(i.e., diferenciable, excepto en el origen) y acotado que permite estabilizar un punto de equilibrio en tiempo finito (esto último, debido a la no diferenciabilidad en el origen). Las estimaciones al conjunto controlable vienen definidas por una familia de elipsoides a un parámetro $\tau$ (que representa una medida del tiempo que se requiere para "llegar" al origen), y se obtienen como solución de una ecuación diferencial matricial no lineal. Además, el control resultante es de tipo subóptimo, i.e., es una aproximación suave al control de tiempo óptimo (que usualmente es discontinuto). Vía un rediseño del control (en una vecindad del punto de equilibrio), obtenemos un control que estabiliza asintóticamente (i.e., en tiempo infinito). En comparación con el control saturado-asintótico y el de tiempo óptimo, el control diseñado presenta lo mejor de ambos: la "suavidad" del primero y la rapidez del último. Particularizado al sistema lineal denominado $n$-integrador, resolvemos el problema de estabilización global. Para esto último, hallamos una solución analítica explícita de la ecuación diferencial matricial arriba mencionada. Este sistema resulta singularmente interesante debido a que, dado que es (críticamente) inestable a lazo abierto (su único valor propio es el cero, con multiplicidad $n$ ), constituye una especie de "test" para las técnicas de estabilización con controles acotados.

En el Capítulo 3 (o [60]), obtenemos una extensión del problema de asignación de valores propios para los sistemas lineales considerando controles acotados. Con base en la teoría LQ (linear-quadratic) construmos una retroalimentación acotada y estabilizante, cuya ganancia depende del tamaño del estado (ganancia programada). Para este fin, se define una familia parametrizada de elipsoides, pero a diferencia con el enfoque del Capítulo 2, esta familia se obtiene resolviendo una familia $\tau$-parametrizada de ecuaciones algebraicas (o diferenciales) matriciales de Riccati. Mediante este método, se construye una función RCA que permite: (i) EGA de los sistemas lineales ACOCA; y (ii) para los sistemas lineales controlables cuyas dinámicas libres son inestables, da lugar a una estimación interna de la región de atracción.

Vale la pena comentar que en la metodologías [15]-[27]-[62] y [60], los controles están definidos de forma implícita, i.e., el sistema a lazo-cerrado consiste de un sistema de ecuaciones diferenciales más una ecuación algebraica no lineal. Además, el control es un estabilizador de tipo "lineal" de la forma $u(x)=-K(x) x$, cuya matriz de ganancias $K(x)$-dependiente de los estados (y que se obtiene a partir de ecuación algebraica antes mencionada), ajusta la amplitud de la entrada de acuerdo al tamaño del estado con el objeto de satisfacer las cotas impuestas sobre la entrada. En este sentido, estas dos metodologías pueden concebirse como basadas en ganancias programadas (gain-scheduling).

Usando un enfoque análogo al del Capítulo 3 (o [60]), Wredenhagen-Bélanger [71] diseñaron un control acotado seccionalmente lineal, el cual también está basado en la teoría LQ. Finalmente, el problema de estabilización global fue también abordado por Teel [67] para la clase de sistemas lineales ACOCA, suponiendo además, que los controles pasan por no linealidades de tipo general. Esta técnica está basada en una solución semiglobal que se obtiene a partir de una familia de ecuaciones algebraicas de Riccati de tipo $H_{\infty}$.

Con respecto a los diferentes enfoques de diseño de control mencionados arriba, es importante remarcar los siguientes dos puntos:

- Los controles construidos mediante la metodología basada en ganancias programadas (en tiempo finito) ([27]-[15]) están orientados hacia un buen desempeño, pero éstos sólo pueden ser implementados en algunos sistemas concretos (e.g., el $n$-integrador [62] y [28]), debido a las ecuaciones diferenciales o integrales no lineales matriciales que hay que resolver. Desafortunadamente, las metodologías LQ ([60]-[71]) o $H_{\infty}([67])$ sufren esta misma desven- 
taja, dando lugar a implementaciones prohibitivas (alto costo computacional, traducido principalmente en cálculos con gran consumo de tiempo), implicadas por las ecuaciones algebraicas de Riccati involucradas. Al final del Capitulo 3 (o [60]), se establece que el problema de complejidad puede mejorarse notoriamente, si se emplea una ecuación diferencial de Riccati. Empero, para ciertas aplicaciones esto puede resultar todavía muy "caro", dado que se requiere resolver un sistema de $n+n(n+1) / 2$ ecuaciones diferenciales - $n$ es la dimensión. En ese capítulo, los inconvenientes arriba mencionados pueden concebirse como un precio que hay que pagar a fin de obtener un resultado de asignación de eigenvalores.

- Por otra parte, los controles diseñados mediante el enfoque de saturaciones anidadas ([64][65|) son más fáciles de implementar. En su estado actual, el diseño con esa estrategia de controles globales provistos de un buen desempeño se encuentra todavía bajo estudio. Al parecer, los primeros controles obtenidos suelen dar respuestas con bajo desempeño, dado que fueron construidos para asegurar la estabilidad sin ninguna otra pretención. Empero, desde una perspectiva semiglobal, existen esfuerzos alentadores para hallar mejores diseños mediante la técnica de baja-y-alta ganancia (low-and-high gain) [48] y también de considerar tanto saturaciones en la magnitud como en la derivada de los controles [36].

Consecuentemente, hace falta una metodología para la estabilización global asintótica de los sistemas lineales que tome ventaja sobre los métodos propuestos a la fecha, y que esté caracterizada por una orientación hacia el buen desempeño y una implementación sencilla de los controles.

De esta manera, nuestro estudio final lo hemos enfocado hacia una metodología que, en la medida de lo posible, satisface las especificaciones anteriores y que además, es más "natural" de extrapolar a sistemas no lineales. Consideramos el problema de la estabilización global mediante controles acotados de los sistemas afines que son Lyapunov estables a lazo abierto. Con base en la literatura consultada sobre el tema, todas las metodologías existentes poseen la desventaja de que los controles acotados son bien sea discontinuos (bang-bang) o con un "mal" desempeño (low gain). Por nuestra parte, hemos obtenido un control retroalimentado, "suave" y acotado, basado en la solución a un problema de optimización paramétrica. En este enfoque, a partir de cierto estabilizador global no (necesariamente) acotado $\nu(x)$ se obtiene, mediante determinada función-peso $\tau(x)$ (relacionada con la norma del control $\nu(x)$ ), una función de control acotada $u(x)$ que estabiliza globalmente el sistema. Así,

$$
u(x)=\frac{1}{\tau(x)} \nu(x)
$$

La construcción de tal función $\tau(x)$ se obtiene mediante la solución de un problema de optimización paramétrica. El conjunto de los sistemas que consideramos incluye a las clases importantes de los sistemas lineales y bilineales.

En el Capítulo 4 (o [54]) estudiamos el caso de los sistemas lineales, para los cuales el problema de optimización (mencionado arriba) se resuelve explícitamente si se considera que las entradas de control se hallan restringidas a un elipsoide: $u \in \mathcal{B}_{1}^{2}=\left\{u \in \mathbb{R}^{m}:\|u\|_{S} \leq 1\right\}$, donde $\|u\|_{S}:=\sqrt{u^{\top} S u}$ y $S$ es una matriz definida positiva. La ley de control propuesta incrementa las ganancias de la retroalimentación a medida que la trayectoria controlada converge hacia el origen, con lo cual se garantiza que las cotas sobre el control nunca serán violadas. Como 
característica adicional, se muestra que si el sistema a lazo abierto es Lyapunov estable, el control propuesto proporciona entradas acotadas con una derivada global acotada: $\|d u / d t\|_{s} \leq \kappa$. Finalmente, se desarrolla un resultado de estabilización semiglobal para los sistemas lineales ACOCA. En este caso, se obtiene un control del tipo low-and-high gain. El método se ihustra con la estabilización global de un navegador inercial, y con la estabilización de un modelo no lineal de un puente de carga (crane control system).

La exposición general para sistemas no lineales se reporta en el artículo [55], y conforma el material de Capítulo 5 ( $y$ último). En este caso, la restricción sobre el control se da en términos de $p$-normas: $u \in \mathcal{B}_{r}^{p}=\left\{u \in \mathbb{R}^{m}:\|u\|_{p} \leq r\right\}$, donde $\|u\|_{p}:=\left(u_{1}^{p}+\ldots+u_{m}^{p}\right)^{1 / p}$ y $p$ par. De esta manera, al incrementar $p$ se puede obtener cualquier grado de aproximación (suave) al $r$-hipercubo $m$-dimensional, mismo que surge como restricción en ciertas aplicaciones. Por consiguiente, se obtiene asi una buena utilización del recurso (magnitud) disponible de control. En ese capítulo, se presenta un enfoque de diseño que permite vía un control retroalimentado acotado, la estabilización global asintótica de sistemas afines cuyas dinámicas libres son Lyapunov estables. Con base en las ideas básicas usadas en los Capítulos 3 y 4 , la ley de control (de alta ganancia) que proponemos en ese capítulo, incrementa la ganancia a medida que la trayectoria controlada converge hacia el origen, con lo cual se garantiza que las cotas sobre la entrada no sean excedidas. Grosso modo, el procedimiento consiste en definir cierta función $(\tau)$, de forma tal que sea constante a lo largo de las fronteras de los conjuntos de nivel $c$ de la función de Lyapunov asociada con el sistema a lazo abierto. Entonces, si tomamos esos conjuntos de nivel $c$ en una sucesión de tamaño decreciente (que converge al origen), se asigna a cada conjunto tal la mayor ganancia posible correspondiente (vía $\tau$ ), bajo la condición de que el control se mantenga acotado. En general, ese procedimiento implica que las ganancias, como funciones de la posición, se obtienen de la solución de un problema de programación no lineal c-parametrizado.

Con especial- interés se estudia una clase importante de sistemas homogéneos (que a su vez incluye a los sistemas que pueden ser estabilizados global y asintóticamente por retroalimentaciones lineales), y sistemas bilineales. Respecto a la primera clase, la ley de control construida está explícitamente definida. En el caso general no lineal (e.g., los sistemas bilineales no homogéneos), el sistema a lazo cerrado resultante está implícitamente definido, en el sentido de que consiste de un sistema de ecuaciones diferenciales más una ecuación algebraica no lineal (requerida para obtener el control). El control diseñado es una función de retroalimentación suave, excepto en el origen (que es una singularidad). No obstante que para um amplio espectro de aplicaciones, ésta es una característica indeseable (e.g., causa del llamado chattering del control), un rediseñamiento del control permite obtener un estabilizador continuo asintótico.

Dependiendo de algunas aplicaciones, e.g. robot manipuladores, procesos químicos de control, etcetera, el considerar que la razón de cambio sobre el control es ilimitada puede concebirse como una desventaja de la mayoría de lo métodos existentes para el diseño de estabilizadores acotados: el control por lo regular semeja un control tipo bang-bang, especialmente cuando los estados se hallan lejos del origen. Ese comportamiento excluye su uso en las mencionadas aplicaciones, debido a las respuestas naturales (e.g. inercia del actuador) de estos sisternas a estímulos externos. A este respecto, en ese capítulo abordamos también el problema de entradas de control sujetas a cotas globales en sus derivadas, para el caso de los sistemas homogéneos antes mencionados. 


\section{ESTABILIZACIÓN DE SISTEMAS LINEA- LES CONTROLABLES CON CONTROLES SUBÓPTIMOS}

\subsection{Introducción}

Los sistemas lineales retroalimentados con entradas acotadas exhiben un comportamiento que podemos calificar de no lineal. La estabilización de estos sistemas constituye un problema de control sumamente interesante. Metodológicamente, este problema se ha abordado usualmente mediante la construcción de una retroalimentación lineal (global) que estabiliza al sistema, para Iuego considerar su saturación (consulte, por ejemplo [12], [58]). En vista del extenso material sobre la teoría de sistemas lineales, éste es un enfoque natural. Sin embargo, es de esperarse que puedan hallarse 'mejores' soluciones al considerar controles no lineales más generales. En particular, para el sistema denominado $n$-integrador, con $n \geq 3$, se ha probado que ninguna retroalimentación lineal saturada puede estabilizarlo globalmente. Empero, este sistema, y en general cualquier sistema lineal cuyos eigenvalores tengan parte real no positiva son globalmente estabilizables mediante un control continuo y acotado [57]. Estas consideraciones nos llevan a cuestionar lo siguiente: ¿será posible diseñar a priori (i.e., no partiendo de una retroalimentación lineal), una función de control continua y acotada la cual nos proporcione un mejor comportamiento a lazo cerrado (en el sentido de aumentar el tamaño de la región de estabilidad y al mismo tiempo obtener una respuesta rápida) que aquél que se obtiene con un control lineal saturado? Hay dos metodologías principales para el diseño de controles continuos y acotados: la basada en la estabilidad asintótica en el sentido de Lyapunov [57], [65], [72] y la basada en estabilidad en tiempo finito [15], [27], [28]. En concreto, en [65] se diseñó una técnica de control continuo y acotado (el enfoque de saturaciones anidadas) para la estabilización global del $n$-integrador, el cual fue extendido en [72] al caso de los sistemas lineales con eigenvalores con parte real no positiva. Por otra parte, se desarrollaron dos enfoques principales para diseñar funciones de control (continuas, excepto en el origen) y acotadas para estabilizar sistemas lineales en tiempo finito. En [15], con base en el método de funciones de controlabilidad [28], se logró estabilizar en tiempo finito el caso de los sistemas lineales con eigenvalores con parte real no positiva. Con el segundo enfoque [27], se obtiene una aproximación al control de tiempo óptimo, usando para ello una estimación interna [7]-[26] a los conjuntos controlables de los sistemas lineales. Debido a que este último método es aplicable a cualquier sistema lineal (autónomo o no), la globalidad de la estabilización sólo puede ser comprobada en casos particulares. En estos dos últimos enfoques, las funciones de control son cle la forma:

$$
u(x)=k^{\top}(x) x, \quad u \in\left[u^{-}, u^{+}\right]
$$


(donde $(\cdot)^{\top}$ denota transposición), los cuales son singulares en el origen (i.e., cuando $x \rightarrow 0$, $k^{\top}(x)$ diverge). En un amplio rango de aplicaciones esto constituye una característica indeseable que debe evitarse para prevenir el denominado "chicoteo" (chattering) del control.

En este capítulo, mostraremos que el control subóptimo (2.1) de [27] puede rediseñarse (fijando las ganancias $h^{\top}(x)$ en una región apropiada (vecindad) del origen), con el objeto de estabilizar asintóticamente. desde una perspectiva local, cualquier sistema lineal controlable. (Empleando la misma idea, en [15] se establece implícitamente que cualquier sistema lineal cuyos eigenvalores tengan parte real no positiva es global y asintóticamente estabilizable). La técnica aquí presentada es aplicada al $n$-integrador, para el cual probamos que el control continuo y acotado resultante es un estabilizador asintótico y global, diferente de los diseñados en [28] y [65]. En la Sección 2.5, mostramos que puede hallarse una solución al problema del control continuo y acotado para los sistemas en el plano. Una extensión de esta técnica fue usada en [53] para estabilizar un(a) (clase de) reactor(es) químico(s) en torno a un punto de equilibrio inestable.

\subsection{Preliminares de control óptimo geométrico}

Consideremos el sistema lineal controlable

$$
\dot{x}=A x+b u, \quad x \in \mathbb{R}^{n},|u| \leq 1
$$

Definición. El conjunto controlable $\mathcal{C}(\tau)$ al tiempo $\tau>0$ (el conjunto de puntos que pueden ser llevados al origen mediante una función de control admisible $u(\tau)$ en un tiempo $\tau$ ) viene dada por

$$
\mathcal{C}(\tau)=\left\{-\int_{0}^{\tau} \exp (-A s) \operatorname{bu}(s) d s: u \in \mathcal{L}^{1}[0, \tau],|u| \leq 1\right\}
$$

donde $\mathcal{L}^{1}[0, \tau]$ es el espacio de funciones reales integrables en el sentido de Lebesgue. El conjunto controlable $\mathcal{C}$ está definido por

$$
\mathcal{C}=\bigcup_{\tau \geq 0} \mathcal{C}(\tau)
$$

Es bien sabido (e.g., Lee-Markus [32]) que $\mathcal{C}(\tau)$ es un conjunto compacto, convexo, simétrico respecto al origen y (que considerado como una función valuada en conjuntos) es continuo en el sentido de Hausdorff. Con base en la propiedad de controlabilidad, se tiene que

$$
\mathcal{C}\left(\tau_{1}\right) \subset \operatorname{int} \mathcal{C}\left(\tau_{2}\right), \quad \text { si } \tau_{1}<\tau_{2}
$$

Introduzcamos la función de tiempo mínimo

$$
\Upsilon_{m}(x)=\inf \{\tau \geq 0: x \in \mathcal{C}(\tau)\}
$$

y denotemos su gradiente por $\nabla \Upsilon_{m}(x)$. Bajo el supuesto de que $\nabla \Upsilon_{m}(x)$ exista en una vecindad abierta del origen, la derivada do $\Upsilon_{m}$ respecto al tiempo, a lo largo de trayectorias de tiempo 
óptimo $x(t)$ de (2.2), satisface (Hajek [18] y Lee-Markus [32])

$$
\dot{\Upsilon}_{m}(x(t))=\left\langle\nabla \Upsilon_{m}(x), A x(t)+b u(t)\right\rangle \equiv-1
$$

donde $u(t)$ es el control de tiempo óptimo correspondiente y $\langle\cdot, \cdot\rangle$ denota el producto interno. $\Upsilon_{m}(x)$ es una función de Lyapunov de tiempo finito, cuya retroalimentación de tiempo óptimo asociada está dada por

$$
u_{m}(x)=-\operatorname{signo}\left\langle\nabla \Upsilon_{m}(x), b\right\rangle .
$$

La construcción y aplicación de este control óptimo implica dos problemas (Hajek [18] y Yeung [73]):

(i) $\Upsilon_{m}(x)$ carece de las propiedades deseadas de diferenciabilidad y

(ii) su determinación requiere conocer el conjunto controlable $\mathcal{C}(\tau)$ en su evolución respecto al tiempo.

Este último aspecto constituye un problema abierto sumamente difícil. Con el objeto de circundar estos problemas, construiremos un control de tiempo finito subóptimo basándonos en una aproximación interna del conjunto $\mathcal{C}(\tau)$ en la línea de los trabajos de Chernous'ko [7] y Komarov [26]-[27].

Definición. Una familia de conjuntos subcontrolables para (2.2) es cualquier función valuada en conjuntos, $\mathcal{V}(\tau)$, tal que $\mathcal{V}(\tau) \subset \mathcal{C}(\tau)$ para toda $\tau \geq 0$, y $\mathcal{V}\left(\tau_{1}\right) \subset \mathcal{V}\left(\tau_{2}\right)$ si $\tau_{1} \leq \tau_{2}$. El conjunto subcontrolable correspondiente está dado por $\mathcal{V}=\cup_{\tau \geq 0} \mathcal{V}(\tau)$.

Consideremos la familia de elipsoides $\tau$-parametrizados siguientes

$$
\mathcal{E}(\tau)=\left\{x \in \mathbb{R}^{n}:\left\langle Q^{-1}(\tau) x, x\right\rangle \leq 1\right\}
$$

donde $Q(\tau)$ es una matriz simétrica definida positiva, la cual varía 'suavemente' respecto al parámetro $\tau$. La existencia de una familia elipsoidal de conjuntos subcontrolables para (2.2) está caracterizada como la solución al problema de Cauchy para la ecuación (2.10) dada a contimuación.

Teorema 2.1. Sea $Q(\tau)$ una solución de la ecuación diferencial matricial con condición inicial

$$
\begin{aligned}
& \frac{d Q}{d \tau}=-A Q-Q A^{\top}+2 F(Q), \quad Q(0)=0 \\
& F(Q)=Q\left(Q^{-1} b b^{\top}\right)^{1 / 2}
\end{aligned}
$$

Entonces, $Q(\tau)$ es una matriz simétrica y definida positiva, $y \mathcal{E}(\tau)$ es una familia de conjuntos subcontrolables para (2.2).

La ecuación (2.10) proporciona la mejor aproximación interna local (en el sentido de volumen máximo) al conjunto controlable $\mathcal{C}(\tau)$ de (2.2) mediante el uso de elipsoides (Chernous'ko [7]). Para simplificar la manipulaciones algebraicas asociadas con la construcción del control, hemos reemplazado la expresión

$$
F(Q)=Q^{1 / 2}\left(Q^{-1 / 2} b b^{\top} Q^{-1 / 2}\right)^{1 / 2} Q^{1 / 2}
$$


obtenida en [26]-[27] por la expresión equivalente (2.10) dada en Solís [52].

Introduzcamos ahora la siguiente función de tiempo mínimo

$$
\Upsilon(x)=\inf \{\tau \geq 0: x \in \mathcal{E}(\tau)\},
$$

la cual es una aproximación a la función de tiempo mínimo $\Upsilon_{m}(x)$ (i.e., $\Upsilon_{m}(x) \leq \Upsilon(x)$ ). El gradiente $\nabla \Upsilon(x)$ está definido para toda $x \in \mathcal{E}=\cup_{\tau \geq 0} \mathcal{E}(\tau)$.

Proposición 2.2. [7][26] Sea $Q(\tau)$ una solución de (2.2) para toda $\tau \geq 0$. Entonces $\Upsilon(x)(=\tau)$ está definida implícitamente por la ecuación

$$
\left\langle Q^{-1}(\Upsilon(x)) x, x\right\rangle=1
$$

Teorema 2.3. [27] Sea $u(x)$ la retroalimentación no lineal definida por

$$
\begin{aligned}
& b u(x)=K(x) x \\
& K(x)=-F(Q(\Upsilon(x))) Q^{-1}(\Upsilon(x)) .
\end{aligned}
$$

Entonces.

$$
\begin{aligned}
& \text { (i) }|u(x)| \leq 1 \\
& \text { (ii) }\langle\nabla \Upsilon(x), A x+K(x) x\rangle \equiv-1
\end{aligned}
$$

La propiedad (2.14 ii) implica que $\Upsilon(x)$ es una función de Lyapunov de tiempo finito para el sistema a lazo cerrado (2.2)-(2.13). Es decir, cualquier punto en $\mathcal{E}$ puede ser llevado al origen en tiempo finito. Concluyendo, el control definido implícitamente por (2.12)-(2.13) proporciona una solución subóptima al problema de control en tiempo mínimo para el sistema (2.2). Como era de esperarse, la matriz de ganancias $K(x)$ diverge a medida que cualquier trayectoria se aproxima en tiempo finito al origen, pues el sistema a lazo cerrado no es Lipschitz en el origen (ino hay unicidad de la solución respecto a condiciones iniciales!).

\subsection{Estabilizadior asintótico continuo y acotado}

El diseño del estabilizador asintótico continuo y acotado es como sigue. Primero, el control de tiempo finito (2.13) llevará al sistema a una vecindad del origen, que corresponde al interior de un conjunto de nivel (isócrona) de $\Upsilon(x)$. De ahí, una retroalimentación lineal con gananciass compatibles (en un cierto sentido explicitado en contexto) llevará, asintóticamente, el estado hasta el origen.

La proposición siguiente establece una conexión entre los problemas de estabilización en tiempo finito y estabilización asintótica para el sistema (2.2). 
Proposición 3.1. Consideremos el sistema a lazo cerrado (2.2) con el control no lineal y acotado (2.13). Sea $W$ una vecindad abierta del origen tal que para toda $x \in W$ la matriz $A+K(x)$ es estable. Entonces, existe una función continua $\hat{K}(x)$ dada por (2.15), tal que el conjunto subcontrolable $\mathcal{E}$ está dentro de la región de estabilidad asintótica del sistema $\dot{x}=(4+\hat{K}(x)) x$. Además, el control retroalimentado $\hat{u}(x)$ definido por $b \hat{u}(x)=\hat{K}(x) x$ satisface $|\hat{u}(x)| \leq 1$.

Prueba. Partiendo de la continuidad de Hausdorff de $\mathcal{C}(\tau)$ y como $\mathcal{C}(0)=\{0\}$, existe un $\tau^{*}>0$ tal que $\mathcal{C}\left(\tau^{*}\right) \subset W$. Entonces $\mathcal{E}\left(\tau^{*}\right) \subset W$. Observemos que para toda $\tau^{*} \geq 0, \Upsilon(x)$ y $K(x)$ son constantes en la frontera $\partial \mathcal{E}\left(\tau^{*}\right)$ del elipsoide $\mathcal{E}\left(\tau^{*}\right)$. Sea $K^{*}$ el valor de $K(x)$ en tal frontera, y definamos la matriz de ganancias

$$
\hat{K}(x)=\left\{\begin{array}{lll}
K(x), & \text { si } & x \notin \mathcal{E}\left(\tau^{*}\right) \\
K^{*}, & \text { si } & x \in \mathcal{E}\left(\tau^{*}\right)
\end{array}\right.
$$

En primera instancia, el conjunto $\mathcal{E}\left(\tau^{*}\right)$ es un atractor de tiempo finito para el conjunto $\mathcal{E} \backslash \mathcal{E}\left(\tau^{*}\right)$. En efecto, dada cualquier $x_{0} \in \mathcal{E} \backslash \mathcal{E}\left(\tau^{*}\right)$, con base en el Teorema 2.3, existe $t_{1}>0$ tal que la solución de (2.2)-(2.13) con condición inicial $x\left(t_{0}\right)=x_{0}$ satisface que $x\left(t_{1}\right) \in \partial \mathcal{E}\left(\tau^{*}\right)$. Además, si recordamos que $\Upsilon(x)$ mide el tiempo para llegar al origen, y que $\partial \mathcal{E}\left(\tau^{*}\right)=\left\{x \in \mathbb{R}^{n}\right.$ : $\left.\Upsilon(x)=\tau^{*}\right\}$, se sigue que $t_{1}=\tau_{0}-\tau^{*}$ si $x_{0} \in \partial \mathcal{E}\left(\tau_{0}\right)$.

Por (2.14 ii), $\Upsilon(x)$ es una función de Lyapunov, luego las trayectorias de (2.2)-(2.13)-(2.15) apuntan al interior del conjunto $\mathcal{E}\left(\tau^{*}\right)$. De aquí que, $\mathcal{E}\left(\tau^{*}\right)$ es además un conjunto invariante.

Por otra parte, para toda $x \in \mathcal{E}\left(\tau^{*}\right)$, el sistema $(2.2)-(2.13)-(2.15)$ coincide con $\dot{x}=\left(A+K^{*}\right) x$. Por hipótesis, $A+K^{*}$ es una matriz estable, luego $x(t) \longrightarrow 0$ asintóticamente, cuando $t \rightarrow \infty$.

Por consiguiente, $\mathcal{E}$ está contenido en la cuenca de estabilidad asintótica del sistema $\dot{x}=$ $(A+\hat{K}(x)) x$.

Por último, tenemos que para toda $x \in \mathcal{E}\left(\tau^{*}\right), b \hat{u}(x)=K^{*} x$-es lineal en $x$. De donde, dado que para toda $x \in \partial \mathcal{E}\left(\tau^{*}\right),|\hat{u}(x)| \leq 1$, se sigue que $|\hat{u}(x)|<1$, si $x \in \operatorname{int} \mathcal{E}\left(\tau^{*}\right)$.

Observación 1 . El resultado obtenido mediante la matriz de ganancias $\hat{K}(x)$ puede expresarse en términos de $K(x)$ usando la siguiente función continua y seccionalmente diferenciable

$$
\theta(x)=\left\{\begin{array}{l}
\Upsilon(x), \text { si } \tau^{*}<\Upsilon(x) \leq \tau_{0} \\
\tau^{*}, \quad \text { si } \Upsilon(x) \leq \tau^{*}
\end{array}\right.
$$

debido a que $\hat{K}(x)=K(\theta(x))$. Para obtener el control acotado y continuo, (2.16) puede usarse para rediseñar el control (2.13) en la vecindad $\mathcal{E}\left(\tau^{*}\right)$ del origen.

Proposición 3.2. Sea $Q(\tau)$ una solución de (2.10). Si $d Q / d \tau$ es una matriz definida positiva en $\left[0, \tau^{*}\right]$ para alguna $\tau^{*}>0$, entonces $A+K^{*}$ es una matriz estable, donde $K^{*}=K(x)$ para toda $x \in \mathcal{E}\left(\tau^{*}\right)$.

Prueba. Definamos la función tipo Lyapunov $V(x)=\left\langle Q^{-1}\left(\tau^{*}\right) x, x\right\rangle$. A partir de (2.12), se sigue que

$$
\nabla \Upsilon(x)=-2 x^{\top} Q^{-1}(\Upsilon(x))\left\langle\frac{d Q^{-1}(\Upsilon(x))}{d \tau} x ; x\right\rangle^{-1}
$$


Si partimos de que $\nabla V(x)=2 x^{\top} Q^{-1}\left(\tau^{*}\right)$, obtenemos

$$
\nabla V(x)=-\left\langle\frac{d Q^{-1}(\Upsilon(x))}{d \tau} x, x\right\rangle \nabla \Upsilon(x),
$$

para toda $x \in \partial \mathcal{E}\left(\tau^{*}\right)$. Consideremos la hipótesis de que $d Q / d \tau$ es una matriz definida positiva. entonces de (2.14), obtenemos que

$$
\dot{V}(x)=\nabla V(x)\left(A+K^{*}\right) x=\left\langle\frac{d Q^{-1}\left(\tau^{*}\right)}{d \tau} x, x\right\rangle<0,
$$

para toda $x \in \partial \mathcal{E}\left(\tau^{*}\right)$. Debido a que $\mathcal{E}\left(\tau^{*}\right)$ es un elipsoide centrado en el origen, tenemos que para toda $y \in \mathcal{E}\left(\tau^{*}\right)$, existen $x \in \partial \mathcal{E}\left(\tau^{*}\right)$ y $\alpha \in \mathrm{R}$ tales que $y=\alpha x$. Por lo tanto, $\dot{V}(y)=\alpha^{2} \dot{V}(x)<0$. Esto prueba que $V(x)$ es una función de Lyapunov para el sistema $\dot{x}=\left(4+K^{*}\right) x$.

Los resultados precedentes pueden resumirse en el siguiente teorema.

Teorema 3.3. Supongamos que $d Q / d \tau$ es uma matriz definida positiva en $\left[0, \tau^{*}\right]$ para algún $\tau^{*}>0$. Entonces, las expresiones (2.13)-(2.16) definen un control continuo y acotado el cual lleva asintóticamente el conjunto subcontrolable $\mathcal{E}$ al origen.

La proposición siguiente establece el significado geométrico de la condición impuesta sobre $d Q / d \tau$.

Proposición 3.4. Sea $Q(\tau)$ una solución de (2.10). Entonces $d Q / d \tau$ es ma matriz definida positiva si y sólo si $\mathcal{E}\left(\tau_{1}\right) \subset \mathcal{E}\left(\tau_{2}\right)$, para $\tau_{1}<\tau_{2}$.

Prueba. Definamos $c\langle E, \psi\rangle:=\sup \left\{\langle f, \psi\rangle: f \in E, \psi \in \mathbb{R}^{n}\right\}$, la función soporte de un conjunto $E$. Supongamos que $\tau_{1}<\tau_{2}$ y que $\psi \in \mathbb{R}^{n} \backslash\{0\}$. Entonces, tenemos las siguientes equivalencias: $\mathcal{E}\left(\tau_{1}\right) \subset \mathcal{E}\left(\tau_{2}\right) \Longleftrightarrow c\left\langle\mathcal{E}\left(\tau_{1}\right), \psi\right\rangle<c\left\langle\mathcal{E}\left(\tau_{2}\right), \psi\right\rangle \Longleftrightarrow 0<\left\langle Q\left(\tau_{1}\right) \psi, \psi\right\rangle^{1 / 2}<\left\langle Q\left(\tau_{2}\right) \psi, \psi\right\rangle^{1 / 2} \Longleftrightarrow$ $\left\langle\left(Q\left(\tau_{2}\right)-Q\left(\tau_{1}\right)\right) \psi, \psi\right\rangle>0 \Longleftrightarrow Q\left(\tau_{2}\right)-Q\left(\tau_{1}\right)$ es una matriz definida positiva $\Longleftrightarrow d Q / d \tau$ es definida positiva, dado que $\tau_{1}$ y $\tau_{2}$ son arbitrarios.

Ahora explotemos la hipótesis de controlabilidad del sistema (2.2) con el objeto de simplificar más la expresión (2.13). Consideremos la siguiente forma canónica la cual es equivalente a cualquer sistema lineal controlable bajo un cambio de coordenadas $(z=\Phi x)$ (Wonham [69|)

$$
\dot{z}=\left(\begin{array}{ccccc}
0 & 1 & 0 & \cdots & 0 \\
0 & 0 & 1 & \cdots & 0 \\
& & & & 1 \\
a_{1} & a_{2} & a_{3} & \cdots & a_{n 2}
\end{array}\right)+\left(\begin{array}{c}
0 \\
\vdots \\
0 \\
1
\end{array}\right) u, \quad|u(x)| \leq 1 .
$$

Lema 3.5. Consideremos una matriz simétrica y definida positiva $Q$ y un vector $U^{\top}=(0, \ldots, 0,1)$, entonces

$$
F(Q)=Q\left(Q^{-1} b b^{\top}\right)^{1 / 2}=\left(\frac{\operatorname{det} Q}{\alpha_{n n}}\right)^{1 / 2} b b^{\top}
$$

donde $Q=\left(q_{i j}\right)$, det $Q$ es el determinante de $Q$ y $\alpha_{n n}$ es el menor principal de $Q$ correspondiente al elemento $q_{n n}$. 
Prueba. A partir de la fómula $Q^{-1}=\operatorname{adj} Q / \operatorname{det} Q$, tenemos

$$
Q\left(Q^{-1} b b^{\top}\right)^{1 / 2}=\frac{1}{\sqrt{\operatorname{det} Q}} Q\left(\operatorname{adj} Q b b^{\top}\right)^{1 / 2}
$$

La función matricial $C^{1 / 2}=\left(\operatorname{adj} Q b b^{\top}\right)^{1 / 2}$ puede obtenerse mediante el polinomio de Lagrange-Sylvester asociado $p(\lambda)=\lambda^{n-1}\left[\alpha_{n n}\right]^{3 / 2-n}$, pues $b b^{\top}=\operatorname{diag}(0, \ldots, 0,1)$ (Gantmacher [13]). Entonces, $C^{1 / 2}$ está dado por

$$
p(C)=C^{1 / 2}=C^{n-1}\left[\alpha_{n n}\right]^{3 / 2-n}=\left[\alpha_{n n}\right]^{-1 / 2} C .
$$

La sustitución de esta expresión en $F(Q)$ proporciona (2.21).

En la siguiente observación resumimos la construcción del control continuo y acotado propuesto.

Observación 2. Sustituyamos la expresión (2.21) en (2.13) y retomemos (2.12) para obtener: el control retroalimentado, de ganancias variables, y de tiempo finito subóptimo

$$
\begin{aligned}
& u(z)=k^{\top}(z) z, \quad z=\Phi x \\
& k^{\top}(z)=-\left(\alpha_{n 1}(z), \ldots, \alpha_{n n}(z)\right) / \sqrt{\operatorname{det} Q(\Upsilon(z)) \alpha_{n n}(z)} \\
& \left\langle Q^{-1}(\Upsilon(x)) x, x\right\rangle=1 \\
& \frac{d Q}{d \tau}=-A Q-Q A^{\top}+2\left(\frac{\operatorname{det} Q}{\alpha_{n n}}\right)^{1 / 2} b b^{\top}, \quad Q(0)=0
\end{aligned}
$$

donde $\alpha_{n i}$ es el menor principal de $Q$ que corresponde al elemento $q_{n i}$, para $i=1, \ldots, n$. Para obtener el control continuo y acotado que resuelve el problema de estabilización asintótica con restricciones, reemplacemos la función $\Upsilon(z)$ en $(2.24 b, c)$ por la función $\theta(z)$, definida en (2.16). El control obtenido en (2.24) es implícito en el sentido que se requiere resolver algebraicamente $(2.24 c)$ on-line para $\Upsilon$.

\subsection{Estabilización global asintótica del $n$-integrador}

En esta sección, especializamos los resultados anteriores al caso del $n$-integrador

$$
x^{(n)}=u, \quad|u(x)| \leq 1,
$$

o de manera equivalente, al del sistema $(2.20)$ con $a_{i}=0(i=1, \ldots, n)$ :

$$
\dot{x}=\left(\begin{array}{ccccc}
0 & 1 & 0 & \cdots & 0 \\
0 & 0 & 1 & \cdots & 0 \\
& & & & 1 \\
0 & 0 & 0 & \cdots & 0
\end{array}\right)+\left(\begin{array}{c}
0 \\
\vdots \\
0 \\
1
\end{array}\right) u, \quad|u(x)| \leq 1
$$


En los artículos [12] y [63] ha sido probado que, para $n \geq 3$, no existe control lineal saturado bajo el cual el sistema (2.26) pueda estabilizarse globalmente. Por otra parte, es posible obtener la estabilización global mediante controles no lineales y acotados más generales [57]. En [65] se diseñó una función de control para este objeto. Basándonos en los resultados de la sección anterior, diseñaremos un control continuo y acotado descrito por (2.24) para estabilizar globalmente al sistema (2.26). El control que obtendremos será una aproximación 'suave' al control de tiempo óptimo.

Sea $Q=\left(q_{i j}\right)$. Para el sistema (2.26), la ecuación diferencial matricial $(2.24 d)$ puede representarse como el siguiente conjunto de $n(n+1) / 2$ ecuaciones diferenciales

$$
\begin{array}{ll}
\frac{d q_{i n}}{d \tau}=-q_{i+1 n} & \text { para } 1 \leq i \leq n-1 \\
\frac{d q_{i j}}{d \tau}=-q_{i+1 j}-q_{i j+1} & \text { para } 1 \leq i \leq j-1 \\
\frac{d q_{r n}}{d \tau}=2\left(\frac{\operatorname{det} Q}{\alpha_{n n}}\right)^{1 / 2} & \text { y } 2 \leq j \leq n-1
\end{array}
$$

Lema 4.1. Una solución analítica y no trivial del sistema $(2.27)$ en la singularidad $Q(0)=0$ (i.e., bajo las condiciones iniciales $q_{i j}(0)=0$ ) está dada por

$$
q_{i j}(\tau)=b_{i j} \tau^{2(n+1)-i-j} \quad \begin{array}{ll}
\text { para } 1 \leq i \leq j \\
y \quad 1 \leq j \leq n
\end{array}
$$

donde cada $b_{i j}$ es un número racional.

Prueba. Como solución para (2.27) proponemos (2.28), considerando a los números $b_{i j}$ como coeficientes indeterminados.

Debido a que grado $\left(q_{i j}(\tau)\right)=\operatorname{grado}\left(q_{i j+1}(\tau)\right)+1$, a la simetría de la matriz $Q$ y a la elección de $q_{n n}(\tau)$, la expresión det $Q / \alpha_{n n}$ es el polinomio cuadrático $p(\tau)=\alpha \tau^{2}$, con $\alpha \in \mathbb{P}$.

Partiendo de los integradores iterados (2.27), los coeficientes $b_{i j}$ son proporcionales entre sí. y podemos escribirlos en términos de $b_{n n}$, i.e.

$$
b_{i j}=\gamma_{i j} b_{n n}, \text { con } \gamma_{i j} \in \mathbb{Q} .
$$

Entonces, el coeficiente $\alpha$ está dado por $\alpha=\gamma b_{n n}$, para $\gamma \in \mathbb{Q}$.

Por último, si sustituimos estas expresiones en la ecuación $(2.27 b)$ obtenemos que $b_{n n}=$ $\gamma \in \mathbb{Q}$. Por lo tanto, los coeficientes $b_{i j}$, para $1 \leq i, j \leq n$, son números racionales.

Con base en que la matriz $Q$ es simétrica y definida positiva, podemos garantizar que existe una matriz racional constante $B=\left(b_{i j}\right)(1 \leq i, j \leq n)$ tal que

$$
\begin{aligned}
& \text { (i) } \operatorname{det} Q=\beta \tau^{n(n+1)} \\
& \text { (ii) } \alpha_{i j}=\beta_{i j} \tau^{n(n+1)-2+i+j} \text {, para toda } \tau>0
\end{aligned}
$$

donde, $\beta=\operatorname{det} B$ y $\beta_{i j}$ es el menor principal de $B$ correspondiente a $b_{i j}$, para $1 \leq i, j \leq n$.

Lema 4.2. El elemento $b_{n n}$ está dado por $b_{n n}=2 / n(n+1)$. 
Prueba. Sea $v(\tau)$ el volumen del elipsoide $\mathcal{E}(\tau)$. Entonces, $v(\tau)=\omega_{n}(\operatorname{det} Q)^{1 / 2}$, donde $\omega_{n}$ es el volumen de la bola unitaria $\mathcal{E}(I)$ ( $I$ es la matriz unitaria). Usando la fórmula

$$
\frac{d \ln (\operatorname{det} S(\tau))}{d \tau}=\operatorname{traza}\left(S^{-1}(\tau) S^{\top}(\tau)\right)
$$

para cualquier familia de matrices invertibles $S(\tau)$ que varían 'suavemente' $\operatorname{con} \tau$, la ecuación siguiente fue obtenida en [46]:

$$
\frac{d \ln (v(\tau))}{d \tau}=\operatorname{traza} A+\operatorname{traza}\left(\left(Q^{-1 / 2}(\tau) b b^{\top} Q^{-1 / 2}(\tau)\right)^{1 / 2}\right)
$$

Si partimos de la prueba del Lema 3.5, obtenemos

$$
\frac{d \ln (v(\tau))}{d \tau}=\operatorname{traza}\left(Q^{1 / 2}(\tau)\left(Q^{-1} b b^{\top}\right)^{1 / 2} Q^{-1 / 2}(\tau)\right)=\sqrt{\frac{\alpha_{n n}}{\operatorname{det} Q}}
$$

Entonces, de (2.30), tenemos $\sqrt{\alpha_{n n} / \operatorname{det} Q}=\sqrt{\beta_{n n} / \beta \tau^{2}}$. Si sustituimos $q_{n n}(\tau)=b_{n n} \tau^{2}$ en $(2.27 b)$, obtenemos $b_{n n}=\sqrt{\beta / \beta_{n n}}$. Ahora usemos esta última expresión e integremos (2.33), con lo cual

$$
v(\tau)=k \tau^{1 / b_{n n}}
$$

A partir de (2.30) tenemos que

$$
v(\tau)=\omega_{n}(\operatorname{det} Q)^{1 / 2}=\omega_{n} \beta^{1 / 2} \tau^{n(n+1) / 2} .
$$

Finalmente, podemos obtener $b_{n n}$ a partir de (2.34) y (2.35).

Sustituyamos (2.30) en (2.24) y tomemos el resultado del Teorema 2.3, para que obtener el control de tiempo finito para el $n$-integrador

$$
\begin{aligned}
& u(x)=k^{\top}(x) x \\
& k_{i}(x)=-c_{i} / \Upsilon^{n-i+1}(x), \quad c_{i}=\beta_{n i} / \sqrt{\beta \beta_{n n}}, \quad \text { para } i=1, \ldots, n
\end{aligned}
$$

donde $\Upsilon(x)$, dada por la relación (2.12), es una función positiva definida en el conjunto subcontrolable $\mathcal{E}$.

Para el $n$-integrador, la prueba del Lema 4.2 sugiere que el control aquí diseñado (2.36) podría ser global, dado que los volúmenes de los elipsoides no son acotados a medida que $\tau \rightarrow \infty$.

Lema 4.3. Para el sistema a lazo cerrado (2.26)-(2.36), tenemos $\mathcal{E}\left(\tau_{1}\right) \subset \mathcal{E}\left(\tau_{2}\right)$, si $\tau_{1}<\tau_{2}$. Además, el conjunto subcontrolable $\mathcal{E}$ es todo $\mathbb{R}^{n}$.

Prueba. De (2.30), tenemos que $Q^{-1}(\tau)(=\operatorname{adj} Q / \operatorname{det} Q)$ está dada por

$$
Q^{-1}(\tau)=\frac{1}{\beta}\left(\begin{array}{ccc}
\beta_{11} \tau^{-2 n} & \cdots & \beta_{\mathrm{In} \tau^{-(n+1)}} \\
\vdots & & \vdots \\
\beta_{n 1} \tau^{-(n+1)} & \cdots & \beta_{n n} \tau^{-2}
\end{array}\right)
$$

La sustitución de (2.37) en la relación (2.12) nos da

$$
\tau^{2 n}-\sum_{i, j=1}^{n} \gamma_{i j} x_{i} x_{j} \tau^{i+j-2}=0, \quad \text { donde } \gamma_{i j}=2 \beta_{i j} / \beta
$$


Ahora, si consideramos (2.38), la intersección de la frontera elipsoidal (2.12) con los ejes coordenados está dada por $x_{i}\left(=\left(0, \ldots, 0, x_{i}, 0, \ldots, 0\right)\right)$, para $i=1, \ldots, n$, donde

$$
x_{i}^{2}=\frac{1}{\gamma(i i} \tau^{2(n-i+1)} \text {. }
$$

Esto implica que $x_{i}<x_{i}^{\prime}$ siempre que $\Upsilon\left(x_{i}\right)<\Upsilon\left(x_{i}^{\prime}\right)$; lo cual significa que $\mathcal{E}\left(\tau_{1}\right) \subset \mathcal{E}\left(\tau_{2}\right)$ si $\tau_{1}<\tau_{2}$. Por último, como $x_{i}$ (intersección) $\rightarrow \infty$, si $\tau \rightarrow \infty$, entonces $\mathcal{E}=\mathbb{R}^{n}$.

Con base en los Lemas 4.2 y 4.3, la expresión (2.36), la Proposición 3.4 y el Teorema 3.3. establecemos el resultado principal de este capítulo en torno al $n$-integrador.

Teorema 4.5. El n-integrador (2.26) es global y asintóticamente estabilizable mediante la función retroalimentada de control

$$
\begin{aligned}
& u(x)=k^{\top}(x) x, \\
& k_{i}(x)=-c_{i} / \theta^{n-i+1}(x), \quad c_{i}=n(n+1) \beta_{n i} / 2 \beta_{n n}, \quad \text { para } i=1, \ldots, n
\end{aligned}
$$

donde los coeficientes $\beta_{n i}$ pueden obtenerse a partir de (2.29)-(2.30) y la función positiva $\theta(x)$ (definida en $\mathbb{R}^{n}$ ) está dada por (2.16).

Observación 3. En [59], se probó que el sistema (2.26) es potencial globalmente estabilizable mediante retroalimentaciones lineales saturadas. Es decir, dado cualquier conjunto acotado $D$, existe un control lineal saturado tal que $D$ está contenido en la región de estabilidad del sistema a lazo cerrado. En particular, se demostró que la región de estabilidad del sistema (2.26) a lazo cerrado con un control lineal saturado, cuya matriz de ganancias es

$$
h^{\top}(\varepsilon)=\left(\varepsilon^{n} c_{1}, \ldots, \varepsilon c_{n}\right)
$$

converge a todo $\mathbb{R}^{n}$ a medicla que $\varepsilon \rightarrow 0$, para ciertas constantes $c_{i}$, para $i=1, \ldots, n$. Debemos remarcar la similitud entre el control obtenido mediante (2.41) y el control diseñado (2.40).

Ejemplo. Para el 3-integrador, tenemos la siguiente retroalimentación

$$
u(x, y, z)=-\left(600^{-3} x+30 \theta^{-2} y+6 \theta^{-1} z\right)
$$

donde, para $\theta=\theta(x, y, z)$, la función $\Upsilon=\Upsilon(x, y, z)$ correspondiente está definida implícitamente por la frontera del elipsoide

$$
\Upsilon^{6}-36 z^{2} \Upsilon^{4}-360 y z \Upsilon^{3}-\left(720 x z+1260 y^{2}\right) \Upsilon^{2}-5760 x y \Upsilon-7200 x^{2}=0
$$

\subsection{Sistemas en el plano}

Consideremos la siguiente familia de sistemas en $\mathrm{R}^{2}$

$$
\left(\begin{array}{l}
\dot{x} \\
\dot{y}
\end{array}\right)=\left(\begin{array}{ll}
0 & 1 \\
a_{1} & a_{2}
\end{array}\right)\left(\begin{array}{l}
x \\
y
\end{array}\right)+\left(\begin{array}{l}
0 \\
1
\end{array}\right) u, \quad|u(x)| \leq 1 .
$$


Para la determinación de la estimación interna $\mathcal{E}(\tau)$ requerimos obtener la solución del siguiente sistema de ecuaciones diferenciales $(2.24 d)$

$$
\begin{aligned}
& \frac{d q_{11}}{d \tau}=-2 q_{12} \\
& \frac{d q_{12}}{d \tau}=-q_{22}-a_{1} q_{11}-a_{2} q_{12} \\
& \frac{d q_{22}}{d \tau}=2\left(-a_{1} q_{12}-a_{2} q_{22}+\sqrt{\frac{q_{11} q_{22}-q_{12}^{2}}{q_{11}}}\right)
\end{aligned}
$$

$\operatorname{con} q_{11}(0)=q_{12}(0)=q_{22}(0)=0$.

Mediante el cambio de coordenadas $\xi=\sqrt{q_{11}}$, y algunas manipulaciones algebraicas, la singularidad (en $\tau=0$ ) es removida, y el sistema (2.42) se transforma en la siguiente ecuación diferencial implícita

$$
\xi \xi^{\prime \prime \prime}+3 \xi^{\prime \prime}\left(\xi^{\prime}+a_{2} \xi\right)+2 \xi \xi^{\prime}\left(a_{2}^{2}-2 a_{1}\right)-2 a_{1} a_{2} \xi^{2}+3 a_{2}\left(\xi^{\prime}\right)^{2}=2 \sqrt{\xi \xi^{\prime \prime}-a_{1} \xi^{2}+a_{2} \xi \xi^{\prime}}
$$

$\operatorname{con} \xi(0)=\xi^{\prime}(0)=0$.

Si la solución de (2.44) existe y es representable como una serie de potencias

$$
\xi(\tau)=d_{2} \tau^{2}+d_{3} \tau^{3}+d_{4} \tau^{4}+d_{5} \tau^{5}+d_{6} \tau^{6}+d_{7} \tau^{7}+o\left(\tau^{8}\right)
$$

podemos obtener los siguientes coeficientes $d_{i}$ (empleando, e.g. Mathematica)

$$
\begin{gathered}
d_{2}=\frac{1}{3 \sqrt{2}}, \quad d_{3}=-\frac{a_{2}}{9 \sqrt{2}}, \quad d_{4}=\frac{30 a_{1}+37 a_{2}^{2}}{3512^{5 / 2}}, \quad d_{5}=-\frac{a_{2}\left(9 a_{1}+5 a_{2}^{2}\right)}{2432^{5 / 2}}, \\
d_{6}=\frac{8316 a_{1}^{2}+24327 a_{1} a_{2}^{2}+8488 a_{2}^{4}}{6160052^{\mathrm{a} / 2}}, \quad d_{7}=-\frac{a_{2}\left(345924 a_{1}^{2}+451353 a_{1} a_{2}^{2}+114652 a_{2}^{4}\right)}{572884652^{9 / 2}} .
\end{gathered}
$$

La solución del sistema (2.43) está dada por

$$
q_{11}=\xi^{2}, \quad q_{12}=-\xi \xi^{\prime} \quad \text { y } \quad q_{22}=\xi \xi^{\prime \prime}+\left(\xi^{\prime}\right)^{2}+a_{2} \xi \xi^{\prime}-a_{1} \xi^{2} .
$$

Observación 4. Como era de esperarse, los primeros coeficientes de la serie de potencias asociada a (2.46) corresponden al 2-integrador, que se obtienen del coeficiente $d_{2}=1 / 3 \sqrt{2}$ (el único que carece de los parámetros $\left.a_{1} \mathrm{y} a_{2}\right)$. Por lo tanto, tenemos que la matriz $d Q / d \tau>0$ en $\left[0, \tau^{*}\right]$, para $\tau^{*}>0$ suficientemente pequeño.

El control retroalimentado de tiempo finito y acotado viene dado por (2.24). Aplicándolo a (2.42), obtenemos el sistema a lazo cerrado siguiente

$$
\begin{aligned}
& \dot{x}=y \\
& \dot{y}=\left(a_{1}+\frac{q_{12}}{\sqrt{\operatorname{det} Q q_{11}}}\right) x+\left(a_{2}-\frac{\sqrt{q_{11}}}{\sqrt{\operatorname{det} Q}}\right) y
\end{aligned}
$$

donde $\operatorname{det} Q=q_{11} q_{22}-q_{12}^{2}$

Dada la condición inicial $(x(0), \theta(0))=\left(x_{0}, \tau_{0}\right), \tau_{0}$ se obtiene como la menor rá́z positiva de (2.12), usando para ello (2.46) y el estado $x_{0}$. Para un sistema inestable a lazo abierto (con $a_{1}=-1$ y $a_{2}=1$ ), en la Figura 1 (arriba) mostramos al conjunto controlable $\mathcal{C}$ (como $\operatorname{Re} \lambda=1 / 2>0$, el conjumto $\mathcal{C}$ es acotado), el conjunto controlable $\mathcal{C}\left(\tau_{0}\right)$ al tiempo $\tau_{0}=2.3$, y su aproximación interna $\mathcal{E}\left(\tau_{0}\right)$. La curva $\alpha(t)$ corresponde a la dinámica a lazo cerrado (2.47) con condición inicial $(0,0.56) \in \partial \mathcal{E}\left(\tau_{0}\right)$. Las correspondientes evoluciones de los estados, el control $\mathrm{y}$ las ganancias se ilustran en la misma figura (abajo). 

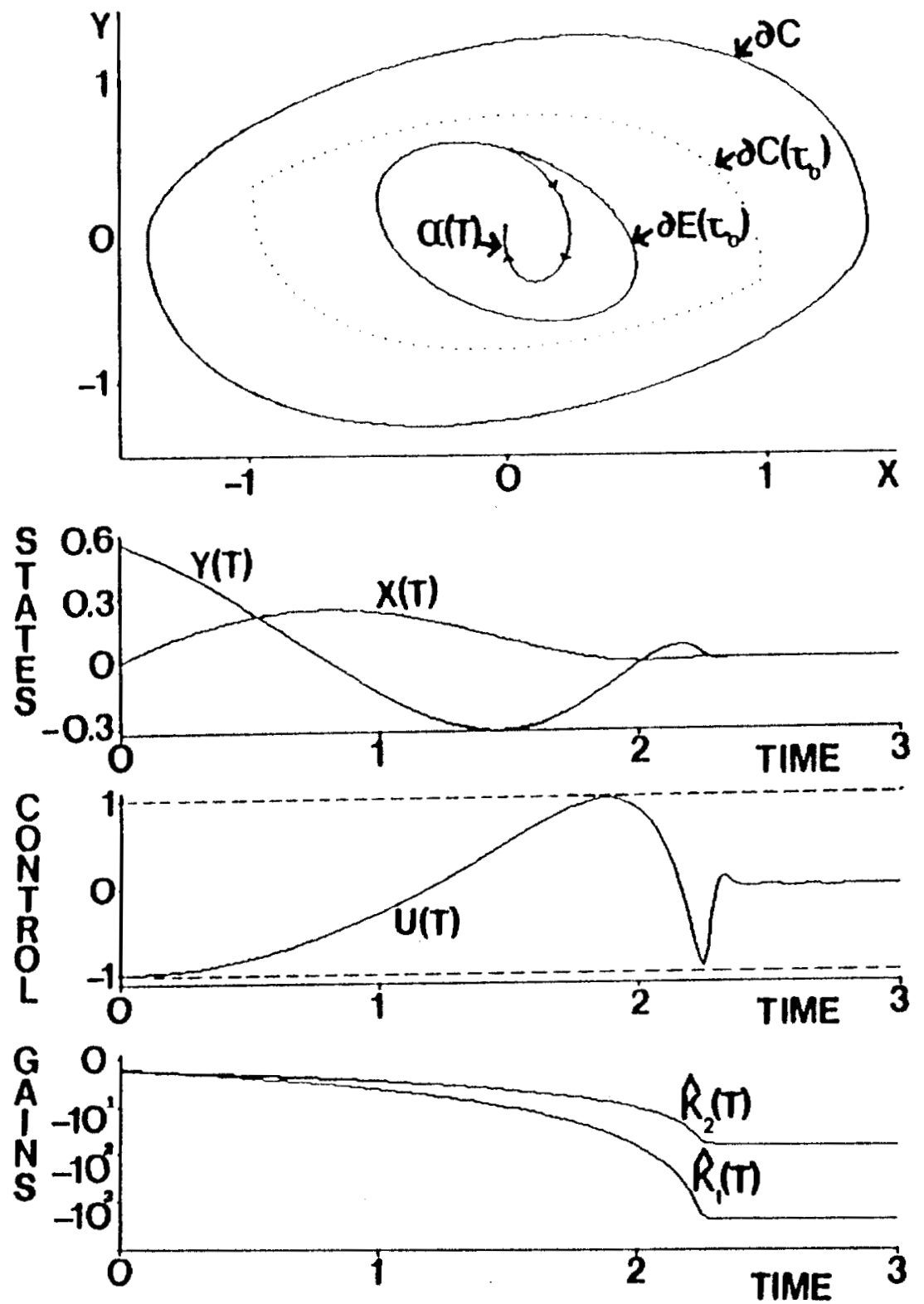

Figura 1 


\section{SISTEMAS LINEALES CON ENTRADAS ACOTADAS: ESTABILIZACIÓN GLOBAL CON ASIGNACIÓN DE EIGENVALORES}

\subsection{Introducción}

La estabilización por retroalimentación de sistemas de control con entradas acotadas es uno de los problemas en la teoría de control más desafiantes. Recientemente, este problema ha atraído mayor interés, especialmente en el caso de los sistemas lineales. Para sistemas lineales, el problema puede ser abordado de dos maneras: Estudiando las propiedades de los controles lineales saturados o diseñando nuevas clases de controles no lineales. Para los controles lineales con saturación, se han estudiado condiciones suficientes para la estabilización global o semiglobal, y la caracterización cualitativa de la región de atracción del origen, desde diferentes puntos de vista por varios autores (por ejemplo [2], [4], [12], [37], [57] y [59]). En particular, hay un gran conjunto de sistemas lineales que pueden ser globalmente estabilizados usando una función continua y acotada, pero que no pueden ser globalmente estabilizados mediante una saturación directa de una retroalimentación lineal ([15] y [57]). Entre los sistemas lineales que no pueden ser globalmente estabilizados con una retroalimentación lineal saturada están los integradores de orden $n(n \geq 3)$ (Fuller [12]). Varios enfoques han sido considerados para afrontar el problema de la estabilización por retroalimentación de sistemas lineales mediante controles no lineales, que son diferentes al de control lineal saturado. El enfoque de Komarov [27] se basa en una estimación interna de los conjuntos controlables por elipsoides. El control retroalimentado se obtiene resolviendo una ecuación diferencial matricial, y es una "aproximación local" al control retroalimentado de tiempo óptimo. Sin embargo, la región donde el control está definido no está bien determinada. En el capítulo anterior (o artículo [62]), resolvimos la ecuación diferential matricial de Komarov para el integrador de orden $n$, probando que el control retroalimentado obtenido es un estabilizador global. En una dirección análoga, partiendo de funciones tipo Lyapunov ('funciones de controlabilidad' [28]), Gavrilyako et al. [15] obtuvieron un control no lineal que, diseñado para sistemas lineales controlables con eigenvalores a lazo abierto con parte real no positiva (PRNP), resulta un estabilizador global. En este caso, la derivación del control retroalimentado implica resolver una ecuación integral (para obtener la 'función de controlabilidad') la cual, en la mayoría de los casos, no es fácil de resolver. Debemos remarcar que los controles de Komarov y Gavrilyako et al. están dados implícitamente (los controles satisfacen una ecuación algebraica no lineal). En los diseños propuestos de los dos enfoques anteriores, la eigenestructura a lazo cerrado está unívocamente definida y no puede ser cambiada. Por otra parte, Yang et al. [72] generalizaron un resultado debido a Teel [65], para probar que los sistemas lineales PRNP pueden ser globalmente estabilizados mediante combinaciones lineales y composiciones anidadas de saturaciones de retroalimentaciones lineales. Recientemente, en 
[64] se obtuvo una mejora de este método, permitiendo además funciones de saturación más generales. Un problema a resolver es cómo obtener asignación de eigenvalores en el diseño de las estrategias de saturación anidada (ver [65]).

Hay al menos dos problemas importantes que no pueden ser resueltos, o no se sabe muy bien cómo abordar, usando los métodos mencionados ([15], [27], [64], [65] y [72]): (i) diseñar un estabilizador global continuo de manera tal que, en una vecindad del origen, el sistema a lazo cerrado tenga sus eigenvalores dentro de cierta región deseada, y (ii) diseñar un control continuo y acotado para sistemas con eigenvalores con parte real positiva. El enfocue que proponemos en este capitulo resuelve ambos problemas.

Este diseño está basado en la siguiente idea geométrica (presentada en [2]). Para un sistema lineal PRNP, existe una familia $\tau$-parametrizada de retroalimentaciones lineales saturadas $u_{\tau}(x)$ que estabilizan asintóticamente el sistema. A medida que el parámetro $\tau$ tiende al infinito. el sistema a lazo cerrado se aproxima al de lazo abierto. En particular, hay (descle un punto de vista dinámico) vecindades elipsoidales invariantes del origen, $\mathcal{E}(\tau)$, que tienden a ser todo $\mathbb{B}^{n}$ cuando $\tau \rightarrow \infty$, y tales que $u_{\tau}$ no se satura en $\mathcal{E}(\tau)$, para cada $\tau \in(0, \infty)$. Por consiguiente, se resuelve el siguiente problema (estabilización semiglobal): Dado un conjunto acotado $D \subset \mathbb{R}^{\prime 2}$, existe $\tau \in(0, \infty)$ tal que el control lineal saturado correspondiente $u_{\tau}(x)$ hace que $D \subset \mathcal{E}(\tau)$. Un inconveniente del enfoque semiglobal consiste en que si se tiene una región de estabilidad grande implica una dinámica lenta (ganancias pequeñas). Estas consideraciones nos conducen a la siguiente pregunta: ¿Cómo variar $\tau$ de manera que tengamos estabilidad global y razones de convergencia rápidas en una vecindad del origen, manteniendo la trayectoria dentro de $\mathcal{E}(\tau)$ para no saturar el control (i.e., diseñar una ley de control a ganancias progtanadas)? Este problema puede ser resuelto diseñando una función dependiente de los estados $\tau(x)$, y proponiendo un control de tipo lineal con ganancias dependientes de los estados de la forma

$$
u(x)=K(\tau(x)) x .
$$

Esta clase de funciones de control fueron consideradas en [15] y [27]. En este capítulo, proponemos la retroalimentación

$$
u(x)=-B^{\top} R(\tau(x)) x,
$$

donde $R(\tau)$ es la solución de una familia $\tau$-parametrizada de (LQR) ecuaciones algebraicas de Riccati. Aquí $(\cdot)^{\top}$ denota transposición.

\subsection{Formulación del problema}

Consideremos el sistema lineal controlable

$$
\dot{x}=A x+B u
$$

donde $x \in \mathbb{R}^{n}, u \in \mathbb{R}^{m}$. Supongamos que el control $u(x)$ está restringido a tomar valores en el conjunto compacto $\mathrm{U}=\mathrm{u}_{1} \times \mathrm{u}_{2} \times \ldots \times \mathrm{u}_{m}$, donde $\mathrm{u}_{i}=\left[-u_{0 i}, u_{0 i}\right], u_{0 i}>0, i=1,2, \ldots, m$.

A fin de simplificar las ideas, a lo largo de esta sección consideremos el caso de entrada escalar, i.e., $u \in\left[-u_{0}, u_{0}\right]$ y $B \in \mathbb{R}^{n}$. 
Existen varios trabajos donde la construcción de un sistema a lazo cerrado con los polos en una región especificada ha sido abordada resolviendo un problema LQR (vea por ejemplo [25] $y[51])$. Sea $Q$ una matriz definida positiva elegida de forma tal que el espectro $\sigma\left(A-B B^{\top} P\right)$ se halla contenido en una región especificada del semiplano izquierdo abierto del plano complejo $\left(\mathbb{C}^{-}\right)$. Aqui $\left(A, Q^{1 / 2}\right)$ es detectable y $P$ satisface la ecuación algebraica de Riccati

$$
A^{\top} P+P A-P B B^{\top} P+Q=0_{n \times n}
$$

Primero observemos que el máximo conjunto (invariante) elipsoidal (definido por la función cuadrática $x^{\top} P x$ ), donde el control $u_{L}(x)=-B^{\top} P x$ no se satura, viene dado por

$$
\mathcal{B E}=\left\{x \in \mathbb{R}^{n}: x^{\top} P x \leq u_{0}^{2} /\left[B^{\top} P B\right]\right\}
$$

Proposición 2.1. BE es el máximo elipsoide definido por la función cuadrática $x^{\top} P x$ donde la función de retroalimentación $u_{L}(x)$ está acotada por $u_{0}$.

Para evitar la saturación del control en la región $\mathbb{R}^{n} \backslash \mathcal{B E}$, la ganancia $-B^{\top} P$ de la retroalimentación lineal $u_{L}(x)$ será modificada como una función de $\tau$. Reescribamos el control $u_{\tau}(x)$ como $-B^{\top} R(\tau) x$ donde $R(\tau)$ es la solución definida positiva de la ecuación de Riccati

$$
A^{\top} R(\tau)+R(\tau) A-R(\tau) B B^{\top} R(\tau)+Q / \tau=0_{n \times n}
$$

De csta manera, proponemos un (LQR) control retroalimentado con ganancias programadas, el cual está acotado por $u_{0}$, y que coincide en $\mathcal{B E}$ con el control lineal $-B^{\top} P x$. El máximo conjunto elipsoidal (invariante), definido por la función cuadrática $x^{\top} R(\tau) x$, donde el control $u_{\tau}(x)=-B^{\top} R(\tau) x$ no se satura está dado por

$$
\mathcal{E}(\tau)=\left\{x \in \mathbb{R}^{n}: x^{\top} R(\tau) x \leq c(\tau)\right\} ; \quad 1 \leq \tau \leq \infty,
$$

donde $c(\tau)=u_{0}^{2} /\left[B^{\top} R(\tau) B\right]$.

Proposición 2.2. $\mathcal{E}(\tau)$ es el máximo elipsoide definido por la función cuadrática $x^{\top} R(\tau) x$, donde la función de control $u_{\tau}(x)$ está acotada por $u_{0}$.

Prueba de las Proposiciones 2.1 y 2.2. Consideremos los valores máximo y mínimo del control $u_{\tau}(x)$ sobre la hipersuperficie $\partial \mathcal{E}(\tau)$. La hipersuperficie $\partial \mathcal{E}(\tau)$ está definida por la igualdad $x^{\top} R(\tau) x-c(\tau)=0$. Para hallar los valores máximo y mínimo de $u_{\tau}(x)$ en $\partial \mathcal{E}(\tau)$, usaremos multiplicadores de Lagrange. La condición necesaria para un extremo es:

$$
2 \bar{x}^{\top} R(\tau)+\lambda B^{\top} R(\tau)=0
$$

que, resolviendo para $\bar{x}$, da $\bar{x}=-\frac{1}{2} \lambda B$. Considerando el hecho de que el peor caso es cuando $-B^{\top} R(\tau) \bar{x}= \pm u_{0}$, obtenemos $\lambda= \pm 2 u_{0} /\left(B^{\top} R(\tau) B\right)$, luego $\bar{x}= \pm B u_{0} /\left(B^{\top} R(\tau) B\right)$. Así, en el extremo se satisfacen las siguientes igualdades

$$
\bar{x}^{\top} R(\tau) \bar{x}=u_{0}^{2} /\left(B^{\top} R(\tau) B\right)=c(\tau) .
$$

Consecuentemente, la retroalimentación $u_{\tau}(x)$ está acotada por $u_{0}$ en $\mathcal{E}(\tau)$. El control $u_{L}(x)$ está acotado en $\mathcal{B E}$ se sigue de que $\mathcal{E}(1)=\mathcal{B E}$. 
Sea $\Re(\tau)=R(\tau) / c(\tau)$, que es una matriz definida positiva para todo $0<\tau<\infty$. Así. redefinamos la familia $\tau$-parametrizada de conjuntos elipsoidales (3.7) como

$$
\mathcal{E}(\tau)=\left\{x \in \mathbb{R}^{n}: x^{\top} \Re(\tau) x \leq 1\right\}
$$

Los siguientes lemas y proposiciones serán usados para probar nuestros resultados principales.

Lema 2.3. (Proposición 3.4 del Capítulo 2) $\Re_{r}=d \Re(\tau) / d \tau$ en una matriz definida negativa si y sólo si $\mathcal{E}\left(\tau_{1}\right) \subset$ int $\mathcal{E}\left(\tau_{2}\right)$, siempre que $\tau_{1}<\tau_{2}$.

Lema 2.4. $\mathrm{i}_{\tau}=d \Re(\tau) / d \tau$ en una matriz definida negativa.

Prueba. Primero demostramos que $R_{\tau}=d R(\tau) / d \tau$ en una matriz definida negativa. Diferenciando ambos lados de (3.6), obtenemos

$$
A^{\top} R_{\tau}+R_{\tau} A-R_{\tau} B B^{\top} R-R B B^{\top} R_{\tau}=Q / \tau^{2}
$$

Denotemos $A_{c}=A-B B^{\top} R(\tau)$. Entonces, la ecuación (3.10) puede ser escrita como

$$
A_{c}^{\top} R_{\tau}+R_{\tau} A_{c}=Q / \tau^{2}
$$

Dado que $\sigma\left(A_{c}\right) \subset \mathbb{C}^{-}$, entonces $R_{\tau}$ en una matriz definida negativa.

Para $\Re_{\tau}=\frac{d}{d \tau} \Re(\tau)=\frac{d}{d \tau}(R(\tau) / c(\tau))$, tenemos

$$
x^{T_{\Re} x}=x^{\top}\left[R_{\tau} c(\tau)-R(\tau) \frac{d c}{d \tau}\right] x / c^{2}(\tau)
$$

Adicionalmente, tenemos la siguiente expresión

$$
d c / d \tau=-u_{0}^{2} B^{\top} R_{\tau} B /\left(B^{\top} R(\tau) B\right)^{2}>0 .
$$

La proposición se sigue de (3.11), porque $R_{\tau}$ en definida negativa.

Proposición 2.5. La familia $\tau$-parametrizada de conjuntos elipsoidales $\mathcal{E}(\tau)$ es un conjunto anidado; es decir, $\mathcal{E}\left(\tau_{1}\right) \subset$ int $\mathcal{E}\left(\tau_{2}\right)$, siempre que $\tau_{1}<\tau_{2}$, con máximo elemento $\mathcal{E}=\cup \mathcal{E}(\tau)$.

Prueba. Se sigue directamente de los Lemas 2.3 y 2.4 .

La idea de tener valores grandes de $\tau$ cuando la trayectoria del sistema está lejos del origen y valores pequeños de $\tau$ cuando se halla cerca del origen (manteniendo la trayectoria dentro $\mathcal{E}(\tau)$ para que el control no se sature) puede resolverse diseñando la siguiente función dependiente de los estados $\tau(x)$.

Definición. Dado $x^{*} \in \mathcal{E}$ y $\tau^{*} \in(1, \infty)$ tales que $x^{*} \in \partial \mathcal{E}\left(\tau^{*}\right)$, definimos $\tau\left(x^{*}\right)=\tau^{*}$ y si $x^{*} \in \mathcal{B E}$, entonces $\tau\left(x^{*}\right)=1$.

Aquí, tomamos $\tau(x)=1$ para toda $x \in \mathcal{B E}$, ya que hemos propuesto una ley no lineal de control que coincide en $\mathcal{B E}$ con el control lineal $-B^{T} P x$. Por contraste, si la función $\tau(x)$ cambia continuamente para toda $x \in \mathbb{R}^{n} \backslash\{0\}$ con $\tau\left(x^{*}\right)=\tau^{*}$ (teniendo también valores en $(0,1)$ ), se obtiene un control de alta ganancia que es continuo pero no es Lipschitz en el origen.

Observemos que si la función $\tau(x)$ se interpreta como una distancia al origen, las "bolas" $\tau(x) \leq r(r$ constante) son los conjuntos elipsoidales $\mathcal{E}(r)$.

Proposición 2.6. $\tau$ es una función continua de $\mathcal{E}$ a $[1, \infty)$, la cual es diferenciable en $\mathcal{E} \backslash \mathcal{B} \mathcal{E}$. 
Prueba. Primero veamos que $\tau(x)$ está bien definida. Observemos que la función $\tau(x)$ es la mínima solución positiva de la ecuación

$$
x^{\top} R(\tau) x-c(\tau)=0 .
$$

Dado $x^{*} \in \mathcal{E}$, existe al menos una $\tau$ tal que $x^{*} \in \mathcal{E}(\tau)$. Definamos $\tau^{*}=\inf \left\{\tau \in[1, \infty): x^{*} \in\right.$ $\mathcal{E}(\tau)\}$

Si $\tau^{*}=1$, entonces $\tau\left(x^{*}\right)=1$. Si $\tau^{*}>1$, entonces $x^{*} \in \partial \mathcal{E}\left(\tau^{*}\right)$. Supongamos que $x^{*} \notin$ $\partial \mathcal{E}\left(\tau^{*}\right)$, o de forma equivalente $x^{* \top} \Re\left(\tau^{*}\right) x^{*}<1$. Así, de la continuidad de $\Re(\tau)$, existe una vecindad de $\tau^{*}$ donde $x^{* \top} \Re(\tau) x^{*}<1$. Esto es una contradicción al hecho que $\tau$ es el ínfimo. Por lo tanto, $x^{* \top} \Re\left(\tau^{*}\right) x^{*}=1$ y $\tau\left(x^{*}\right)=\tau^{*}$. La unicidad de $\tau^{*}$ se sigıe de la Proposición 2.5, pues $\partial \mathcal{E}\left(\tau_{1}\right) \cap \partial \mathcal{E}\left(\tau_{2}\right)=\emptyset$, siempre que $\tau_{1} \neq \tau_{2}$.

En virtud de que $\Re(\tau)$ es continua, la función valuada en conjuntos $\tau \rightarrow \mathcal{E}(\tau)$ es continua en la métrica de Hausdorff. Usando este hecho, es fácil probar que $\tau$ es continua. Por otra parte, si definimos $g(\tau, x)=x^{\top} \Re(\tau) x-1$, entonces $\partial g / \partial \tau=x^{\top} \Re_{\tau} x \neq 0$ para toda $x \in \mathcal{E} \backslash \mathcal{B E}$. Por el Teorema de la Función Implícita, ésta es una condición suficiente para la diferenciabilidad de $\tau(x)$.

\subsection{Resultados principales}

El control retroalimentado $u(x)$, dado por:

$$
u(x)=\left\{\begin{array}{lll}
-B^{\top} R(\tau(x)) x, & \text { si } x \in \mathcal{E} \backslash \mathcal{B E} \\
-B^{\top} R(1) x, & \text { si } x \in \mathcal{B E}
\end{array}\right.
$$

define una ley de control continua no lineal que estabiliza asintóticamente (3.3) en la siguiente manera: Primero, el control no lineal lleva las trayectorias del sistema a una vecindad del origen $\mathcal{B E}$, entonces, la retroalimentación lineal $-B^{\top} R(1) x$ tomará asintóticamente el estado hacia el origen.

Teorema 3.1. Sea $R(\tau)$ una solución definida positiva de la ecuación (3.6), y $\mathcal{E}(\tau)$ el conjunto elipsoidal definido por (3.9). Entonces, la función de control $u(x)$ definida por (3.13) satisface la restricción $-u_{0} \leq u(x) \leq u_{0}$ y lleva cualquier punto $x \in \mathcal{E}=\cup \mathcal{E}(\tau)$ al origen.

Prueba. Recordemos que $\mathcal{B E}=\left\{x \in \mathbb{R}^{n}: x^{\top} R(1) x \leq c(1)\right\}$, luego $\mathcal{B E}$ es un conjunto de nivel invariante de la función de Lyapunov $V(x)=x^{\top} R(1) x$ para el sistema lineal $\dot{x}=(A-$ $\left.B B^{\top} R(1)\right) x$. Consecuentemente, como $u(x)=-B^{\top} R(1) x$ en $\mathcal{B E}$, todas las trayectorias que llegan a $\mathcal{B E}$ son llevadas asintóticamente al origen.

Es suficiente con probar que el conjunto compacto $\mathcal{B E}$ es un atractor de tiempo finito en $\mathcal{E}$, para el sistema a lazo cerrado $\dot{x}=A x+B u(x)$. En $\mathcal{E} \backslash \mathcal{B E}, \dot{\tau}(x)$ se puede calcular de (3.12), y así obtenemos

$$
\dot{\tau}=\frac{-2 x^{\top} R(\tau) \dot{x}}{x^{\top} R_{\tau} x-d c / d \tau}
$$

para toda $x \in \mathcal{E} \backslash \mathcal{B E}$. Entonces, $\mathcal{B E}$ es un atractor si la derivada de $\tau(x)$ a lo largo del campo vectorial a lazo cerrado $\tau<0$, para toda $x$ en la cerradura de $\mathcal{E} \backslash \mathcal{B E}$. En efecto, como 
$2 x^{\top} R(\tau) \dot{x}=x^{\top}\left(A_{c}^{\top} R+R A_{c}\right) x=-x^{\top}\left(Q / \tau+R B B^{\top} R\right) x<0, R_{\tau}$ es definida negativa, y $c(\tau)$ es una función creciente, se sigue de (3.14) que $\tau<0$. Finalmente, el control $u(x)$ leva todos los puntos $x \in \mathcal{E}$ al conjunto $\mathcal{B E}$, y por consiguiente, al origen. Con base en lo anterior y las Proposiciones 2.2 y 2.5 , el teorema queda probado.

En lo que sigue, trataremos el caso general de entradas múltiples (i.e., $m \geq 1$ ). Sea $B=$ $\left[b_{1}, \ldots, b_{m}\right]$ y $c_{i}(\tau)=u_{0 i}^{2} /\left[b_{i}^{\top} R(\tau) v_{i}\right], i=1, \ldots, m$. Definimos

$$
c(\tau)=\min _{i}\left\{c_{i}(\tau)\right\}
$$

Como $c_{i}(\tau), i=1, \ldots, m$, son funciones crecientes de $\tau, \mathrm{c}(\tau)$ es también una función creciente de $\tau$. A pesar de que $c(\tau)$ no es necesariamente diferenciable para toda $\tau>0$, la derivada por: la derecha, $d \mathrm{c} / d \tau=\lim \{(\mathrm{c}(\tau+h)-\mathrm{c}(\tau)) / h: h \rightarrow 0, h>0\}$, está bien definida.

Teorema 3.2. Sea $R(\tau)$ una solución definida positiva de (3.6). Definamos la función $\tau(x)$ en la siguiente forma: para $x \in \mathcal{B E}=\left\{x \in \mathbb{R}^{n}: x^{\top} P x \leq \mathrm{c}(1)\right\}$ y $\tau(x)=1$, y para toda $x \in \mathbb{R}^{n} \backslash \mathcal{B C}$ como la solución positiva de la ecuación

$$
x^{\top} R(\tau) x-\mathrm{c}(\tau)=0
$$

Entonces, el control $u(x)$ definido por

$$
u(x)=\left\{\begin{array}{lll}
-B^{\top} R(\tau(x)) x, & \text { si } & x \in \mathcal{E} \backslash \mathcal{B} \mathcal{E} \\
-B^{\top} P(1) x, & \text { si } & x \in \mathcal{B} \mathcal{E}
\end{array}\right.
$$

satisface las restricciones $-u_{0 i} \leq u_{i}(x) \leq u_{0 i}, i=1, \ldots, m$, y lleva todo punto en $\mathcal{E}$ al origen.

Prueba. La demostración de la estabilidad asintótica con $\mathcal{E}$ contenido en la región de atracción del origen, se sigue por las mismas líneas de la prueba para el caso de entrada escalar, considerando la derivada por la derecha de $c(\tau)$ en lugar de la derivada de $c(\tau)$. De manera análoga al caso de entrada escalar, el problema de probar que $u_{i}(x)$ está acotado por $u_{0 i}$ se reduce al problema de estimar los valores máximo y mínimo de $u_{i}(x)$ en la hipersuperficie $\partial \mathcal{E}(\tau)=\left\{x \in \mathbb{R}^{n}: \tau(x)=\beta\right\}$. Esto lleva a la conclusión que la condición en el peor caso se satisface para

$$
\bar{x}^{\top} R(\beta) \bar{x}=u_{0 i}^{2} /\left[b_{i}^{\top} R(\beta) b_{i}\right]=c_{i}(\beta) .
$$

Entonces, cuando $c(\tau)$ se toma como el mínimo, las cotas $-u_{0 i} \leq u_{i}(x) \leq u_{0 i}$, se satisfacen para toda $i=1, \ldots, m$.

Para estudiar la región invariante de no saturación $\mathcal{E}$ del sistema a lazo cerrado (3.3)-(3.17), debe considerarse el siguiente resultado sobre la solución de la ecuación algebraica de Riccati (3.6). cuando el parámetro $\tau$ diverge a $\infty$.

Lema 3.3. [44] Si todos los eigenvalores de la matriz A tienen parte real no positiva, entonces $R(\infty)=0_{n \times n}$.

Lema 3.4. Consideremos un cambio de base donde $R(\infty)$ se escribe como

$$
h(\infty)=\left[\begin{array}{cc}
0 & 0 \\
0 & W
\end{array}\right]
$$


con $W$ una matriz definida positiva. En esta base, tenemos

$$
A=\left[\begin{array}{cc}
A_{s i} & A_{*} \\
0 & A_{u}
\end{array}\right] \quad B=\left[\begin{array}{c}
B_{s i} \\
B_{u}
\end{array}\right]
$$

donde los eigenvalores de $A_{s i}, A_{u}$ tienen parte real no positiva y positiva, respectivamente. Además $A_{u}-B_{u} B_{u}^{\top} W$ es una matriz estable.

Prueba. El resultado se obtiene siguiendo las ideas dadas en [44].

Teorema 3.5. Si el sistema (3.3) es PRNP, entonces (3.17) es un estabilizador global. Si A tiene $k$ eigenvalores parte real positiva, entonces $\mathcal{E}$ es igual al producto cartesiano de un conjunto elipsoidal (abierto) $k$-dimensional y $\mathbb{R}^{n-k}$. El conjunto elipsoidal es una transformación lineal de $\left\{x_{u} \in \mathbb{R}^{k}: \min _{i}\left[b_{u i}^{\top} W b_{u i}\right] x_{u}^{\top} W x_{u}<u_{0}^{2}\right\}$, conde $W$ fue definido en el Lema 3.4 y $u_{0}=\min _{i}\left\{u_{0 i}\right\}$.

Prueba. Del Lema 3.3, si el sistema es PRNP entonces $R(\tau) \rightarrow 0_{n \times n}$ cuando $\tau \rightarrow \infty$. De donde,

$$
\left\{x \in \mathbb{R}^{n}: \min _{i}\left(\left[b_{i}^{\top} R(\tau) b_{i}\right] / u_{0 i}^{2}\right) x^{\top} R(\tau) x \leq 1\right\}=\mathcal{E}(\tau)
$$

converge a $\mathbb{R}^{n}$, (i.e., (3.17) es un estabilizador global). Por otra parte, del Lema 3.4 hay una transformación de coordenadas tal que $R(\tau)$ puede escribirse como $\left(R_{i j}(\tau): 1 \leq i, j \leq 2\right)$, donde $R_{11}(\tau) \rightarrow 0_{(n-k) \times(n-k)}, R_{12}(\tau)=R_{21}^{\top}(\tau) \rightarrow 0_{(n-k) \times k}$, y $R_{22}(\tau) \rightarrow W$ cuando $\tau \rightarrow \infty$. Por lo tanto, para cualquier

$$
x^{*}=\left(x_{s i}^{*}, x_{u}^{*}\right) \in \mathbb{R}^{n-k} \times\left\{x_{u} \in \mathbb{R}^{k}: \min _{i}\left(\left[b_{u i}^{\top} W b_{u i}\right] / u_{0 i}^{2}\right) x_{u}^{\top} W x_{u}<1\right\}
$$

existe un $\tau^{*}$ suficientemente grande, tal que

$$
\min _{i}\left(\left[b_{i}^{\top} R\left(\tau^{*}\right) b_{i}\right] / u_{0 i}^{2}\right) x^{* \top} \Re\left(\tau^{*}\right) x^{*} \leq 1
$$

donde $\Re(\tau)=R(\tau) / \mathrm{c}(\tau)$. Entonces, $x^{*} \in \mathcal{E}\left(\tau^{*}\right) \subset \mathcal{E}$, y el teorema queda demostrado.

A continuación tenemos algunas observaciones con respecto a extensiones y problemas abiertos relacionados con el problema de estabilización aquí estudiado.

Observación 1. En general, las soluciones de (3.6)-(3.12) no se pueden obtener explícitamente (analíticamente). Así, debemos considerar una aproximación numérica de la solución. Dado que $\mathcal{E}(\tau)$ es una familia anidada de conjuntos elipsoidales, $\left(\mathcal{E}\left(\tau_{1}\right) \subset \operatorname{int} \mathcal{E}\left(\tau_{2}\right)\right.$ si $\left.\tau_{1}<\tau_{2}\right)$, se puede obtener una ley de control seccionalmente lineal resolviendo numéricamente (3.6)-(3.12) para una sucesión de valores del parámetro $\tau, 1=\tau_{1}<\tau_{2}<\ldots<\tau_{r}$, donde $\tau_{r}$ de elige de manera tal que $\mathcal{E}\left(\tau_{r}\right)$ contenga al conjunto de puntos $D$ a estabilizar. Sea $\Delta \mathcal{E}\left(\tau_{j}\right)=\mathcal{E}\left(\tau_{j}\right) \backslash \mathcal{E}\left(\tau_{j-1}\right)$, donde $\mathcal{E}\left(\tau_{0}\right)=\emptyset$. Consideremos la función de retroalimentación definida en el conjunto $\mathcal{E}^{*}=\cup_{j=1}^{r} \mathcal{E}\left(\tau_{j}\right)$,

$$
u_{P L}(x)=-B^{T} R\left(\tau_{j}\right) x \text { si } x \in \Delta \mathcal{E}\left(\tau_{j}\right), \text { para toda } j=1,2, \ldots, r .
$$

Observemos que $\mathcal{E}^{*}$ es una unión finita de conjuntos ajenos, $\mathcal{E}^{*}=\cup_{j=1}^{r} \Delta \mathcal{E}\left(\tau_{j}\right)$, y $u_{P L}$ es lineal en cada conjunto $\Delta \mathcal{E}\left(\tau_{j}\right)$. De donde, el control $u_{P L}(x)$ es una función acotada seccionalmente lineal pero no continua que estabiliza el sistema (3.3) en $\mathcal{E}^{*}$. La estabilización se obtiene del hecho de que las trayectorias convergen al origen para cualquier $\tau_{j}$; entonces $\mathcal{E}\left(\tau_{j-1}\right)$ es un atractor de 
tiempo finito para el flujo definido en $\Delta \mathcal{E}\left(\tau_{j}\right)$. Para evitar cruces tangentiales de las trayectorias controladas en $\partial \mathcal{E}\left(\tau_{j-1}\right)$, la sucesión $1=\tau_{1}<\tau_{2}<\cdots<\tau_{r}$ debe elegirse de manera tal que

$$
x^{\top} R\left(\tau_{j-1}\right)\left(A x-B B^{\top} R\left(\tau_{j}\right)\right)<0 \text { para toda } x \in \partial \mathcal{E}\left(\tau_{j-1}\right),
$$

para un $\Delta \tau_{j}$ suficientemente pequeño. En [71], se usaron procedimientos de diseño análogos para sistemas lineales sujetos a restricciones sobre las entradas de control.

Observación 2. Para resolver el problema de estabilización global, puede usarse también una Ecuación Diferencial de Riccati (EDR) que posee varias ventajas sobre la Ecuación Algebraica de Riccati (EAR). En efecto, en más fácil de resolver numéricamente una EDR que una EAR. Sea $P(\tau)$ la solución de la ecuación

$$
\frac{d}{d \tau} P=P_{\tau}=A^{\top} P(\tau)+P(\tau) A-P(\tau) B B^{\top} P(\tau)
$$

con $P(1)=P$ (la solución de (3.4)). Como $P_{\tau}(1)=-Q<0$, entonces $P_{r}<0$ para todo $\tau \in(1, \infty)$ (vea [47]). Basados en este hecho, tenemos que las Proposiciones 2.2 y 2.5 son válidas (en la definición de los conjuntos elipsoidales (3.7) escribimos $P(\tau)$ en lugar de $R(\tau)$ ). Consecuentemente, $\tau(x)$ está bien definida y los Teoremas 3.1 y 3.2 pueden probarse mediante los mismos argumentos. Además, cuando $\tau$ tiende al infinito, la solición $P(\tau)$ converge a la solución $P_{\infty}$ de la expresión asociada EAR

$$
A^{\top} P_{\infty}+P_{\infty} A-P_{\infty} B B^{\top} P_{\infty}=0
$$

El Teorema 3.5 también puede probarse para $u_{c}(x)=-B^{\top} P(\tau) x$ (vea los Lemas 3.3 y 3.4). En particular, $u_{c}(x)$ es un estabilizador global de (3.3) si el sistema lineal es PNRP.

Observación 3. Notemos que $u(x)$ definido por (3.17) nunca se satura. Esta es una propiedad importante porque un diseño basado en este enfoque evita comportamientos ad versos del control.

\subsection{Ejemplo numérico}

Consideremos el integrador de segundo orden: $\dot{x}=y$ y $\dot{y}=u$. Este sistema tiene al cero como eigenvalor de multiplicidarl dos. Luego, del Teorema 3.1, el sistema puede ser globalmente estabilizado por el control (3.13). Sea $Q=Q^{\top}=\left(q_{i j}\right), i, j=1,2$. Denotemos $q_{1}=q_{11}, q_{2}=q_{12}$ y $q_{3}=q_{22}$. Si resolvemos la ecuación (3.6) para $R(\tau)$, obtenemos

$$
R(\tau)=\left[\begin{array}{cc}
\frac{1}{\tau}\left(\sqrt{q_{1}} \sqrt{2 \sqrt{q_{1} \tau}+q_{3}}-q_{2}\right) & \sqrt{q_{1} / \tau} \\
\sqrt{q_{1} / \tau} & \frac{1}{\sqrt{\tau}} \sqrt{2 \sqrt{q_{1} \tau}+q_{3}}
\end{array}\right] .
$$

Por otra parte,

$$
c(\tau)=\tau u_{0}^{2} /\left[\sqrt{\tau} \sqrt{2 \sqrt{q_{1} \tau}+q_{3}}\right]
$$


Por lo tanto, la ecuación (3.12) es:

$$
\begin{gathered}
{\left[\sqrt{q_{1} \tau}\left(2 \sqrt{q_{1} \tau}+q_{3}\right)-q_{2} \sqrt{\tau} \sqrt{2 \sqrt{q_{1} \tau}+q_{3}}\right] x_{1}^{2}+} \\
{\left[2 \sqrt{q_{1}} \tau \sqrt{2 \sqrt{q_{1} \tau}+q_{3}}\right] x_{1} x_{2}+\left[\tau\left(2 \sqrt{q_{1} \tau}+q_{3}\right)\right] x_{2}^{2}-\tau^{2} u_{0}^{2}=0 .}
\end{gathered}
$$

Esta ecuación puede resolverse numéricamente para $\tau$. En la Figura 1 (a) se muestra el desempeño de las trayectorias cuando se usa el control (3.13) con $q_{1}=25, q_{2}=0, q_{3}=6$ y $u_{0}=1$. En la Figura 1 (a), se muestra también la frontera elipsoidal $\partial \mathcal{B E}=\left\{x \in \mathbb{R}^{2}: \tau(x)=1\right\}$, donde en control continuo (3.13) conmuta de una retroalimentación no lineal a una lineal. En $\mathcal{B E}$, el sistema a lazo cerrado se comporta como uno lineal con eigenvalores fijos $-2 \pm i$. En la Figura 1 (b) se exhibe la acción del control. Esta muestra que $|u(x)| \leq 1$, para todo $t \geq 0$.

Para ilustrar la construcción del control de la Observación 1, la Figura 2 (a) muestra una trayectoria en retrato fase para la sucesión del parámetro $\tau: \tau_{1}=1, \tau_{2}=2, \tau_{3}=3$ y $\tau_{4}=4$. En la Figura 2(b) se exhibe la acción del control para tal trayectoria. Notemos que en este caso el control es discontinuo cuando la trayectoria cruza las fronteras $\partial \mathcal{E}\left(\tau_{j}\right)$.

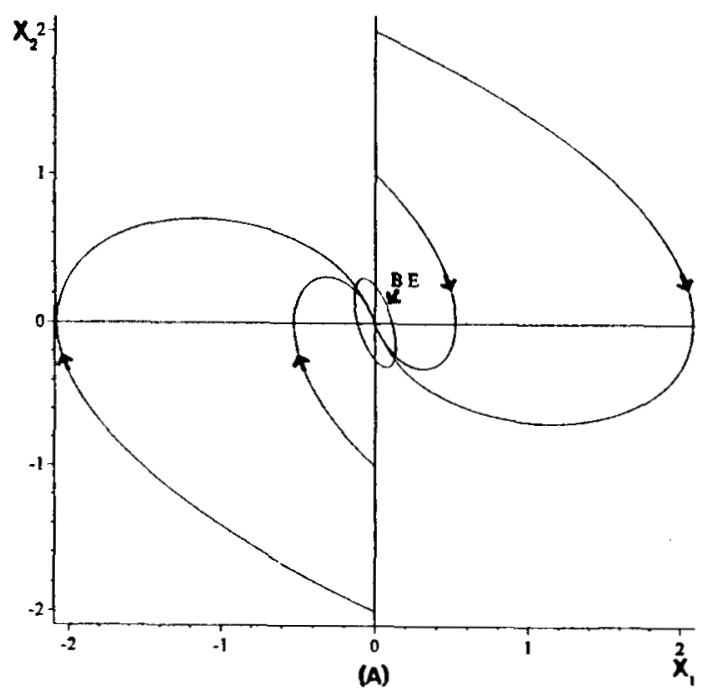

Figura 1 


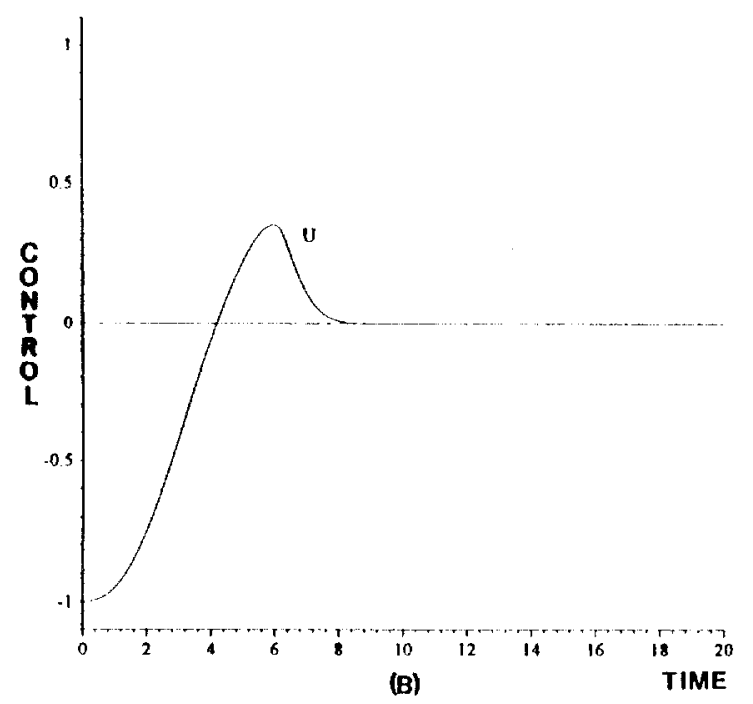

Figure 1.
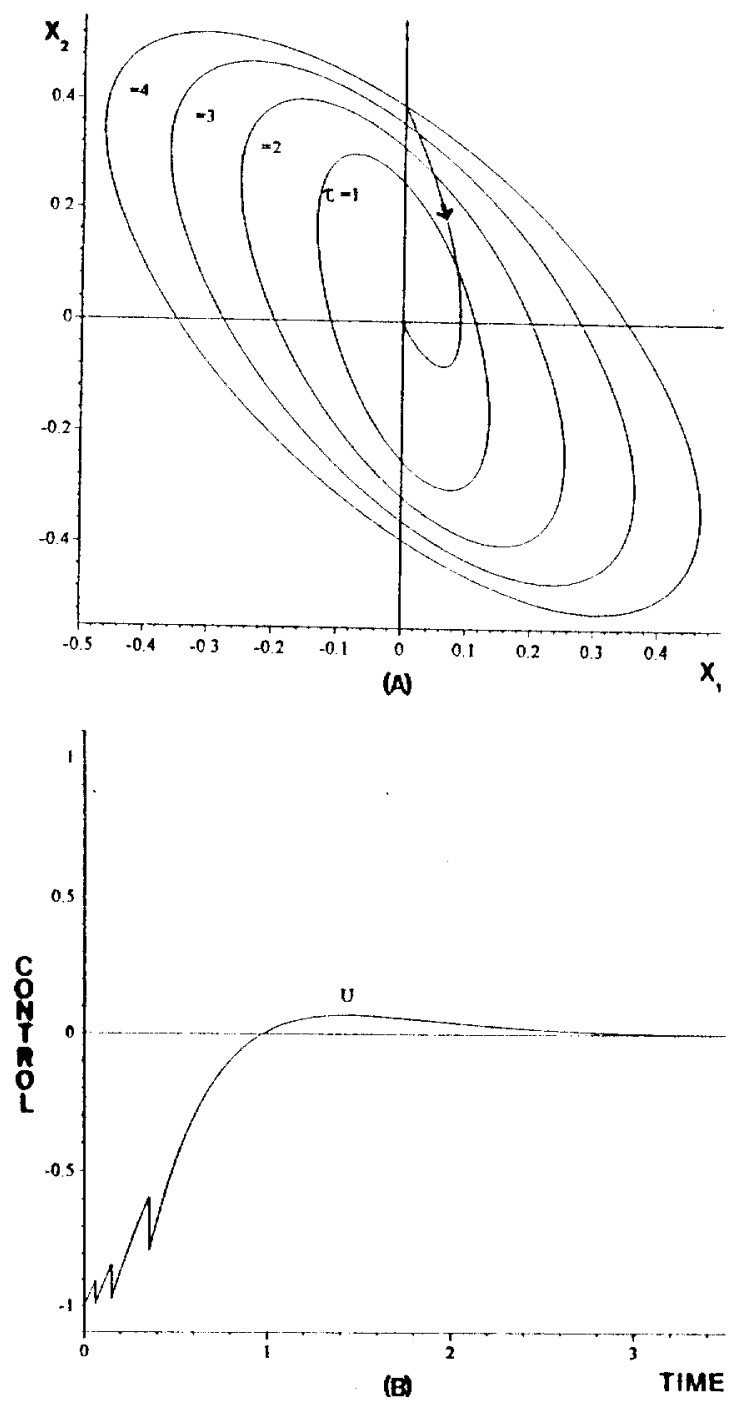

Figura 2 


\section{ESTABILIZACIÓN GLOBAL Y SEMIGLO- BAL DE SISTEMAS LINEALES MEDIANTE CONTROLES ACOTADOS}

\subsection{Introducción}

Consideremos el sistema lineal con entradas múltiples

$$
\dot{x}=A x+B u,
$$

donde $x \in \mathbb{R}^{n}, A$ y $B$, son matrices de dimensiones $n \times n$ y $n \times m$, respectivamente, y la entrada $u$ toma valores en el conjunto compacto (elipsoide) $\mathcal{B}_{1}^{2}=\left\{u \in \mathbb{R}^{m}:\|u\|_{S} \leq 1\right\}$-la bola unitaria $S$-euclideana en $\mathbb{R}^{m}$, i.e. $\|u\|_{S}:=\sqrt{u^{\top} S u}$, donde $S=R^{-2}$ y $R$ es una matriz simétrica definida positiva.

En este capítulo, estudiaremos el problema de la estabilización global asintótica (EGA) del origen de los sistemas (4.1) mediante retroalimentaciones de control acotadas (RCA) $u=u(x)$, sujetas a la restricción $\|u(x)\|_{S} \leq 1$. A lo largo de todo el capítulo, la estabilización siempre será entendida como estabilización respecto al origen.

En los últimos años, ha habido un renovado interés respecto al problema de la estabilización global del sistema (4.1) mediante las RCA. Con el objeto de estudiar el problema anterior, se llegó a la caracterización de una clase de sistemas lineales: Sistema lineal asintóticamente controlable al origen con controles acotados (ACOCA). Es bien sabido, que un sistema lineal es ACOCA sii es estabilizable (i.e., todos sus modos no controlables tienen parte real negativa) y su dinámica libre asociada tiene todos sus eigenvalores con parte real no positiva (PRNP), cf. por ejemplo, Lee-Markus [32], Schmitendorf-Barmish [49] y Sontag [56]. Además, el enfoque natural de considerar saturaciones de retroalimentaciones lineales tiene el inconveniente de que la estabilización global asintótica (EGA) no puede garantizarse a priori. E incluso, en algunos casos una saturación hard puede no llevar a la estabilidad global; en específico, el $n$-integrador $(n \geq 3)$ Fuller [12]. A pesar de este resultado negativo, Sontag-Sussmann [57] probaron que la EGA de los sistema lineales ACOCA puede realizarse mediante la clase general de las funciones RCA. Sin embargo, el diseño de controles en ese trabajo estaba basado en un procedimiento recursivo muy complicado (computacionalmente difícil). Teel [65] introdujo una metodología que empleaba combinaciones lineales y composiciones anidadas de saturaciones de retroalimentaciones lineales (enfoque de saturaciones anidadas) para obtener la EGA del $n$ integrador. Finalmente, Sussmann-Sontag-Yang [64] generalizaron ese método para afrontar el problema anterior, en vista de lo cual obtuvieron la EGA de los sistemas lineales ACOCA, suponiendo además, funciones de saturación más generales.

Una antigua solución al problema anterior, i.e., llevar cualquier estado inicial al origen, en tiempo finito, bajo el supuesto de considerar controles acotados (discontinuos) fue obtenida 
con la teoria de control de tiempo óptimo [32]. Empero, la derivación de funciones de control retroalimentado en tiempo óptimo puede ser muy engorrosa y su subsecuente implementación es muy difícil: se requiere de enormes cantidades de memoria on-line para almacenar hipersuperficies de cambio (switching) intricadas. En otros términos, su construcción necesita del conocimiento de los conjuntos controlables de los sistemas lineales. Este último aspecto plantea, en general, un problema sumamente difícil (abierto). En particular, Korobov-Sklyar. obtuvieron soluciones analíticas al problema de tiempo óptimo para el $n$-integrador [29], y para sistemas con control escalar cuyas dinámicas asociadas tienen eigenvalores puramente imaginarios [30]. Hasta ahora, el papel de la teoría de control de tiempo óptimo se reduce más bien a producir patrones a considerar como metas en sistemas reales: una ayuda para construir sistemas de control subóptimos. Sobre esta base, e interesado en hallar una solución suave al problema de control en tiempo subóptimo, Komarov [27] usó una estimación interna elipsoidal de los conjuntos controlables de sistemas lineales no autónomos. Un parámetro (o más bien una matriz parametrizada) de esa aproximación elipsoidal se obtiene resolviendo una ecuación diferencial matricial no lineal. El estabilizador resultante es una función de control acotada y subóptima de tiempo finito, la cual es suave, excepto en el origen. Sin embargo, la región de atracción no está bien determinada, al igual que falta establecer las condiciones para la existencia de soluciones para esa ecuación diferencial matricial en el origen (una singularidad en este caso). Basados en el enfoque de Komarov, y especializados a sistemas lineales autónomos controlables de una entrada, en el Capítulo 2 (o [62]), establecimos una conexión entre la estabilización asintótica y la de tiempo finito via un rediseñamiento del controlador y obtuvimos un función RCA. Ahí también resolvimos el problema de la EGA para el n-integrador. Para esto último, hallamos una solución analítica explícita de la ecuación diferencial arriba mencionada. Usando un enfoque alternativo, pero no involucrado en obtener una solución subóptima, Fiorobov [28] se abocó al problema de la estabilización en tiempo finito del $n$-integrator, mediante las denominadas funciones de controlabilidad (funciones tipo Lyapunov). Ese método fue extendido por Gavrilyako-Korobov-Sklyar [15] a fin de obtener la estabilidad global en tiempo finito para los sistemas lineales controlables PRNP. Para ello, debe resolverse una ecuación integral no lineal. El problema de hallar soluciones a esta ecuación es una tarea muy laboriosa.

Vale la pena comentar que en la metodología ([15]-[27]-[28]-[62]), los controles están definidos de forma implícita, i.e., el sistema a lazo-cerrado consiste de un sistema de ecuaciones diferenciales más una ecuación algebraica no lineal. Además, el control es un estabilizador de tipo lineal de la forma $u(x)=-K(x) x$, cuya matriz de ganancias $K(x)$-dependiente de los estados (obtenida a partir de ecuación algebraica antes mencionada), ajusta la amplitud de la entrada de acuerdo al tamaño del estado con el objeto de satisfacer las cotas impuestas sobre la entrada. En este sentido, toda esta metodología puede ser concebida como basada en ganancias programadas (gain-scheduling).

En el Capítulo 3 (o [60]), introdujimos una técnica que proporciona un resultado de asignación de eigenvalores para sistemas lineales con controles acotados. Mediante ese método, se construye una función RCA la cual permite: (i) EGA de los sistemas lineales ACOCA: y (ii) para los sistemas lineales controlables cuyas dinámicas libres son inestables, da lugar a una estimación interna de la región de atracción. En ese trabajo, partinos de la teoría LQ para derivar una retroalimentación acotada y estabilizante global, cuya ganancia depende del tamaño del estado (ganancia programada). Para este fin, se debe resolver una familia $\tau$-parametrizada de ecuaciones matriciales algebraicas (o diferenciales) de Riccati con el objeto de calcular el 
control. Finalmente, en Suárez-Álvarez-Sznaier-Ibarra [61], considerando tanto perturbaciones aditivas como control acotado, se obtuvo una función RCA que permite estabilizar global y asintóticamente el sistema a lazo cerrado y una atenuación de perturbaciones $\mathcal{L}^{2}$ en $\mathcal{L}^{2}$ en una vecindad del origen.

Usando un enfoque análogo a [60], Wredenhagen-Bélanger [71] diseñaron una control acotado seccionalmente lineal, el cual también está basado en la teoría LQ. Finalmente, el problema de estabilización global fue también abordado por Teel [67] para la clase de sistemas lineales ACOCA, suponiendo además, que los controles pasan por no linealidades de tipo general. Esta técnica está basada en una solución semiglobal que se obtiene a partir de una familia de ecuaciones algebraicas de Riccati de tipo $H_{\infty}$.

Con respecto a los diferentes enfoques de diseño de control mencionados arriba, es importante remarcar los siguientes dos puntos:

- Los controles construidos mediante la metodología basada en ganancias programadas (en tiempo finito) ([27]-[28]) están orientados hacia un buen desempeño, pero éstos sólo pueden ser implementados en algunos sistemas concretos (e.g., el $n$-integrador [62]), debido a las ecuaciones diferenciales o integrales no lineales matriciales que hay que resolver. Desafortunadamente, las metodologías LQ ([60]-[71]) o $H_{\infty}([61\}-[67])$ sufren esta misma desventaja, dando lugar a implementaciones prohibitivas (alto costo computacional, traducido principalmente en cálculos con mucho consumo de tiempo), implicadas por las ecuaciones algebraicas de Riccati involucradas. Al final del Capítulo 3 (o [60]), se establece que el problema de complejidad puede mejorarse notoriamente, si se emplea una ecuación diferencial de Riccati. Empero, para ciertas aplicaciones esto puede resultar todavía muy "caro", dado que se requiere resolver un sistema de $n+n(n+1) / 2$ ecuaciones diferenciales $-n$ es la dimensión. En ese capítulo, los inconvenientes arriba mencionados pueden concebirse como un precio que hay que pagar a fin de obtener un resultado de asignación de eigenvalores.

- Por otra parte, los controles diseñados vía el enfoque de saturaciones anidadas ([64]-[65]) son más fáciles de implementar. En su estado actual, el diseño en esa estrategia de controles globales con buen desempeño se encuentra todavía bajo estudio. Al parecer, los primeros controles obtenidos suelen dar respuestas con bajo desempeño, dado que fueron construidos para asegurar la estabilidad sin ninguna pretención de obtener un buen desempeño. Empero, desde una perspectiva semiglobal, existen esfuerzos alentadores para hallar mejores diseños mediante la técnica de baja-y-alta ganancia (low-and-high gain) [48] y también de considerar tanto saturaciones en la magnitud como en la derivada de los controles [36].

Consecuentemente, hace falta una metodología para la estabilización global asintótica de los sistemas lineales que tome ventaja sobre los enfoques propuestos a la fecha, y que esté caracterizada por una orientación hacia el buen desempeño y una implementación sencilla de los controles.

En este capítulo, basado en [60]-[62], construimos una función RCA para la estabilización de sistemas lineales cuyas dinámicas libres son estables en el sentido de Lyapunov. La ley de control propuesta, incrementa las ganancias de la retroalimentación a medida que la trayectoria 
controlada converge hacia el origen. Esto garantiza que nunca se excederán las cotas sobre el control. Se define una sucesión de conjuntos invariantes de tamaños decrecientes, asociados con una función de Lyapunov, y relacionamos a cada uno la correspondiente ganancia más alta posible, bajo la restricción de que el control se mantenga acotado. Las ganancias como funciones de la posición se derivan explícitamente resolviendo un problema de programación c-parametrizado. Dependiendo de algunas aplicaciones, e.g. robot manipuladores, procesos químicos de control, etcetera, el considerar que la razón de cambio sobre el control es ilimitada puede concebirse como una desventaja de la mayoría de lo métodos existentes para el diseño de estabilizadores acotados: el control por lo regular semeja un control tipo bang-bang, especialmente cuando los estados se hallan lejos del origen. Ese comportamiento excluye su uso en las mencionadas aplicaciones, debido a las respuestas naturales (e.g. inercia del actuador) de estos sistemas a estímulos externos. Comparado con esos métodos, una cualidad remarcable de nuestra técnica recae en la "suavidad" de la derivada de los controles. Además, se muestra que si el sistema a lazo abierto es estable en el sentido de Lyapunov, el control propuesto proporciona entradas acotadas con una derivada global acotada: $\|d u / d t\|_{S} \leq \kappa$. Finalmente, se desarrolla un resultado de estabilización semiglobal para los sistemas lineales ACOCA. En este caso, se obtiene un control tipo low-and-high gain. El método se ilustra con la estabilización global de un navegador inercial, $y$ con la estabilización de un modelo no lineal de un puente de carga (loading bridge).

\subsection{Planteamiento del problema}

Supondremos que el sistema (4.1) satisface la siguiente hipótesis.

Hipótesis H1. Supongamos que existe una matriz definida positiva y simétrica $P\left(=P^{\top}>0\right)$, que es solución de la ecuación de Lyapunov

$$
A^{\top} P+P A=-Q
$$

donde $Q$ es un matriz semidefinida positiva $(Q \geq 0) y(\cdot)^{\top}$ denota transposición. Entonces, la función cuadrática

$$
V(x)=\frac{1}{2} x^{\top} P x
$$

define una función de Lyapunov para el sistema a lazo abierto (4.1).

La hipótesis $\mathrm{H} 1$ establece que la matriz $A$ es Hurwitz o marginalmente estable.

Observación 1. Hay una gran cantidad de sistemas prácticos interesantes que pertenecen a la clase de sistemas contemplados por la hypothesis $\mathrm{H} 1$. La mayoría de los procesos químicos prácticos son ejemplos importantes de ello. En particular, las columnas de destilación son sistemas de múltiples entradas de control que son estables a lazo abierto, pero las especificaciones de producción y rechazo a perturbaciones requieren de una dinámica a lazo cerrado "rápida" bajo la restricción natural de acciones de control acotadas [45].

Consideremos un sistema extendido entrada-salida compuesto del sistema (4.1) con una salida artificial

$$
y=C x=B^{\top} P x
$$


Además de la hipótesis H1, el sistema (4.1)-(4.4) se supone que satisface la siguiente hipótesis.

Hipótesis H2. El sistema de entrada-salida (4.1)-(4.4) es detectable.

Con base en esto, podemos establecer el siguiente resultado.

Proposición 2.1. Supongamos que el sistema (4.1) satisface las hipótesis H1 y H2. Entonces, la función de control

$$
u(x)=-\rho B^{\top} P x, \quad \rho>0,
$$

es un estabilizador global asintótico para el sistema (4.1).

Esbozo de la prueba. Con base en la hipótesis $\mathrm{H} 1, V(x)$, y $\rho>0$, tenemos que $d V / d t$ es semidefinida negativa. Si $N:=\left\{x \in \mathbb{R}^{n}: d V / d t=0\right\}$, entonces es un subespacio (lineal); de forma que $x=0$ es un punto de equilibrio estable del sistema a lazo cerrado. Además, dentro de $N$ las trayectorias a lazo cerrado coinciden con las de lazo abierto, pues $\left.u(x)\right|_{N} \equiv 0$. Entonces, $N$ es un subespacio $A$-invariante. Por otra parte, en virtud de la hipótesis $\mathrm{H} 2$, tenemos que $A$ es estable en el subespacio no observable $U=\left\{x \in \mathbb{R}^{n}: C A^{i} x=B^{\top} P A^{i} x=0,0 \leq i \leq n-1\right\}$, que contiene a $N$. Así, $x=0$ es un punto de equilibrio global asintóticamente estable.

Observación 2. En [5], se obtuvo una generalización de la proposición anterior para sistemas afines no lineales en términos de pasividad y una extensión natural de detectabilidad.

Hasta ahora, el control dado en (4.5) no es acotado. Sin embargo, de la hipótesis H1 tenemos que la matriz $A$ tiene su espectro localizado en el semiplano cerrado izquierdo del piano complejo, $\sigma(A) \subset \mathrm{cl}\left(\mathbb{C}^{-}\right)$, lo cual implica que los sistemas bajo consideración son sistemas lineales ACOCA (i.e., estabilizables mediante entradas arbitrariamente pequeñas). Por lo tanto, uno puede usar una saturación suave para obtener una función globalmente RCA (cf. [2]), para esta clase de sistemas lineales. En particular, Lin [35] propuso la siguiente modificación al control (4.5)

$$
\begin{aligned}
& u(x)=-\rho(x) B^{\top} P x, \\
& \rho(x)=\frac{2 r}{1+\left\|B^{\top} P x\right\|_{I}^{2}}
\end{aligned}
$$

la cual proporciona una function RCA (4.6), tal que $\|u(x)\|_{I} \leq r$, para cualquier $r>0$ (aquí, $\|\cdot\|_{I}$ es la norma euclideana usual). El control (4.6) estabiliza global asintóticamente el sistema lineal (4.1) [35]. Resulta importante observar que este control es del tipo de baja ganancia (low-gain), i.e., la estabilización global asintótica se obtiene a expensas de variaciones lentas del control. Los controles de baja ganancia pueden dar respuestas "perezosas" y un bajo desempeño para todas las condiciones iniciales, incluso aquellas cercanas al origen.

Una manera de obtener un control de alta ganancia consiste en aumentar la función $\rho(x)$ a medida que la distancia al origen se reduce, de forma tal que el control pueda usar la máxima amplitud de la entrada sin violar las restricciones sobre la misma. El objetivo de este capitulo es proveer una metodología para diseñar la ganancia del control como una función de la posición (ganancia programada), de tal manera que se utilice toda la magnitud control disponible. La idea central es incrementar $\rho(x)$ a medida que la trayectoria se aproxima al origen. En nuestro diseño, $\rho(x)$ es constante a lo largo de los conjuntos de nivel de la función de Lyapumov asociada al sistema a lazo abierto. En el capítulo anterior, usamos un enfoque de diseño de control de ganancias programadas (LQ) para probar que los sistemas lineales ACOCA pueden ser globalmente estabilizados. 


\subsubsection{Ideas geométricas preliminares}

Con el objeto de aclarar las ideas geométricas sobre las que está basado nuestro enfoque de diseño, presentamos la estabilizacion de (4.1) mediante una retroalimentación escalar (4.5) con un parámetro constante $\rho>0$. Luego, motivados por el resultado, en la siguiente sección mostraremos cómo construir una función de los estados $p(x)$, que proporciona una función RCA y buenas razones de convergencia para el sistema a lazo cerrado.

Luego, si suponemos que el control es escalar: $B=b \in \mathbb{R}^{n}$ y entonces $S=r^{-2}>0$. Definimos el conjunto (no vacío)

$$
U(\rho)=\left\{x \in \mathbb{P}^{n}:-r \leq-\rho b^{\top} P x \leq r\right\},
$$

tal que $0 \in \operatorname{int} U(\rho)$. Las fronteras de $U(\rho)$ están dadas por los hiperplanos

$$
\begin{aligned}
& H_{ \pm}=\left\{x \in \mathbb{R}^{n}: \gamma^{ \pm}(x)=0\right\}, \\
& \gamma^{ \pm}(x):=-b^{\top} P x \pm \frac{r}{\rho} .
\end{aligned}
$$

Como $\partial \gamma^{+} / \partial \rho>0$ y $\partial \gamma^{-} / \partial \rho<0$, entonces es evidente que

$$
U\left(\rho_{2}\right) \subset U\left(\rho_{1}\right), \quad \text { para } \rho_{1}<\rho_{2} .
$$

Si consideramos la saturación del control (4.5) para $\rho>0, U(\rho) \subset \mathbb{R}^{n}$ es la región donde el control no se satura. Si $\rho$ crece, entonces la región de no saturación del control $U(\rho)$ decrece. mientras que si $\rho$ tiende a cero, tenemos

$$
\lim _{\rho \rightarrow 0} U(\rho)=\mathbb{R}^{n}
$$

y se recupera la dinámica del sistema a lazo abierto.

Denotemos al (elipsoide) conjunto de nivel $c(c>0)$ de la función de Lyapunor $V(x)$ considerada en la hipótesis $\mathrm{H} 1$,

$$
\mathcal{E}(c)=\left\{x \in \mathbb{R}^{n}: V(x) \leq c\right\}
$$

tal que $0 \in \operatorname{int} \mathcal{E}(c)$.

Como $U(\rho)$ es no vacio, existe un conjunto máximo $\mathcal{E}\left(c^{*}\right)$. donde $c^{*}$ depende de $\rho$. contenido en $U(\rho)$. El conjunto $\mathcal{E}\left(c^{*}\right)$ es invariante bajo las trayectorias del sistema a lazo cerrado (4.1)(4.5), luego todas las trayectorias que empiezan en $\mathcal{E}\left(c^{*}\right)$ convergen asintóticamente a 0 . mientras que el control queda acotado por $r$. De esta manera, $\mathcal{E}\left(c^{*}\right)$ es un estimado de la región de atracción del origen, $\Omega(0)$, basado en la función de Lyapunov $V(x)$. Además, es fácil verificar que

$$
\dot{V}=-\frac{1}{2} x^{\top} Q x-\rho\left(b^{\top} P x\right)^{2} \leq-\alpha \rho
$$

donde $\alpha=\max _{x}\left(b^{\top} P x\right)^{2}$, para $x \in \mathcal{E}\left(c^{*}\right)$ (pues $V(x)$ es propia). Para mejorar la convergencia. debemos incrementar el valor de $\rho$. Sin embargo, debido a (4.9) el valor de $c^{*}(\rho)$ se reduce (y por ende, el tamaño de $\left.\mathcal{E}\left(c^{*}\right)\right)$. Por el contrario, si $\mathcal{E}\left(c^{*}(\rho)\right)$ es mayor, las trayectorias convergerín más lento. Aquí surge el dilema: ¿Cómo diseñar el parámetro $r$ como función de la posición a fin de obtener una buena razón de convergencia y estabilidad global con un control acotado? 


\subsection{Un problema de programación paramétrica}

Cualquier $x_{0} \in \mathbb{R}^{n}$ puede llevarse asintóticamente al origen mediante una elección adecuada del parámetro $p$ en el control (4.5). En efecto, sólo se requiere buscar un valor de $c=c(\rho)$ tal que

$$
x_{0} \in \mathcal{E}(c) \subset U(\rho) .
$$

En virtud de que $\mathcal{E}(c)$ es un conjunto de nivel (invariante) de $V(x)$, la trayectoria $x_{t}\left(x_{0}\right)$ convergerá asintóticamente al origen. Por otra parte, la inclusión $\mathcal{E}(c) \subset U(p)$ asegura que el control $u(x)=-\rho b^{\top} P x$ no se saturará para toda $t \geq 0$. Además, toda condición inicial puede llevarse al origen mediante (4.5) debido a (4.10). En este sentido, podemos decir que el sistema (4.1) es semiglobamente estabilizable por (4.5) con $\rho$ constante, i.e., para cualquier $x_{0} \in \mathbb{R}^{n}$, podemos elegir $\rho>0$ tal que $x_{0} \in \Omega(0)$-la región de atracción del origen. Empero, con este enfoque, el sistema es semiglobalmente estabilizado a expensas de un control de baja ganancia.

Resumiendo la discusión en curso, y retomando el caso de múltiples entradas, el mejor diseño semiglobal consiste en elegir el mayor valor de $\rho$ de forma tal que el control $u(x)=-\rho B^{\top} P x$ nunca se sature a lo largo de la trajectoria $x_{t}\left(x_{0}\right)$, i.e., dado $x_{0}$ se elige $\rho$ de manera que

$$
c^{*}=V\left(x_{0}\right)
$$

y $\mathcal{E}\left(c^{*}\right)$ es el mayor conjunto de nivel contenido en $U(\rho)$. Este problema puede plantearse como el problema de programación

$$
\begin{gathered}
\max \rho \geq 0 \\
\text { t.q. } \quad \mathcal{E}(c) \subset U(\rho) .
\end{gathered}
$$

Con el objeto de simplificar la presentación, definamos $\tau=1 / p$. Entonces, el problema anterior puede parafrasearse como

$$
\begin{array}{ll} 
& \min \tau \geq 0 \\
& \left\|-B^{\top} P x\right\|_{S} \leq \tau \\
\text { t.q. } & \\
& V(x) \leq V\left(x_{0}\right),
\end{array}
$$

Dado que $V(0)=0<V\left(x_{0}\right)$, para $x_{0} \neq 0$, es fácil ver que si existe una solución $\rho^{*}=1 / \tau^{*}$ âl problema (4.16), entonces $\mathcal{E}\left(c^{*}\right)=\mathcal{E}\left(V\left(x_{0}\right)\right) \subset U\left(\rho^{*}\right)$. Así, $\rho^{*}$ será el mayor valor de $\rho$ que permite la convergencia asintótica al 0 de la trayectoria $x_{t}\left(x_{0}\right)$ con la máxima razón de convergencia respecto a la función de Lyapunov $V(x)$, y al mismo que el control $u(x)=-\rho^{*} B^{\top} P x$ permance acotado, para toda $t \geq 0$. Consideremos las condición (de frontera) $u \in \partial U$, i.e. $\tau=\left\|-B^{\top} P x\right\|_{S}$, para obtener la siguiente formulación equivalente de (4.16)

$$
\begin{gathered}
\tau\left(x_{0}\right)=\max _{x}\left\|-B^{\top} P x\right\|_{S} \\
\text { t.q. } x \in \partial \mathcal{E}(c), \quad \text { donde } c=V\left(x_{0}\right) .
\end{gathered}
$$

El problema de optimización (4.17) es una familia de prograrnas 1-paramétricos $(c \geq 0)$ con una restricción de igualdad variable [10]. En particular, como $V(x)$ es propia (por la hipótesis H1), 
la familia de programas dada arriba es propia [10]. Un programa propio (como (4.17)) tiene al menos una solución global para cada $c \in \mathbb{R}$, pues el conjunto $V^{-1}(c)(=\partial \mathcal{E}(c))$ es compacto.

Definamos el control $\tau$-dependiente

$$
u_{\tau}(x):=-\frac{1}{\tau(x)} B^{\top} P x
$$

Definición. Una solución $\tau=\tau(x)$ del problema de optimizaciôn (4.17) se clenominará admisible ssi satisface las siguientes propiedades:

(a) $\tau(x)$ es definida positiva;

(b) $\tau(x)$ es una función suave, con la posible excepción de $x=0 ; y$

(c) la igualdad $S_{\tau^{*}}=\left\{x \in \mathbb{R}^{n}: \tau(x) \leq \tau^{*}\right\}=\mathcal{E}\left(c\left(\tau^{*}\right)\right)$ se satisface, para cada $\tau^{*} \geq 0$. Así, $S_{\tau^{*}}$ es un conjunto invariante bajo las trayectorias del sistema a lazo cerrado (4.1)-(4.18).

Para el sistema (4.1), probaremos (abajo) que cualquier solución de (4.17) es de hecho admisible.

Si $\tau(x)$ es una solución admisible, el control $u_{\tau}(x)(4.18)$ es suave excepto en el origen, donde es singular (i.e., no está definido en $x=0$ ). Luego, el origen es más bien una singularidad en lugar de un punto de equilibrio del sistema a lazo cerrado (4.1)-(4.18). Esto significa que este sistema no es Lipschitz en el origen (no unicidad de las soluciones respecto a condiciones iniciales), así todas las trayectorias podrían converger a $x=0$ en un tiempo finito. En caso de que esta característica pudiera llevar a un comportamiento indeseable, e.g. causar el llamado "chicoteo" (chattering) del control, esta ley de control puede redefinirse para obtener la siguiente función RCA asintótica (diferenciable excepto en $\partial \mathcal{E}\left(\tau^{\times}\right)$)

$$
u^{\times}(x)=\left\{\begin{array}{ll}
u_{\tau}(x), & \text { si } x \in \mathbb{R}^{n} \backslash \mathcal{E}\left(\tau^{\times}\right) \\
-\frac{1}{\tau^{\times}} B^{\top} P x, & \text { si } x \in \mathcal{E}\left(\tau^{\times}\right)
\end{array},\right.
$$

donde $\tau^{\times}>0$ es arbitrario, que estabiliza asintóticamente al sistema lineal $(4.1)(c f . \quad[60]$ y [62]).

Proposición 3.2. Con base en las hipóthesis $\mathrm{H} 1$ y $\mathrm{H} 2$, si $\tau(x)$ es una solución admisible del problema de optimización (4.17), entonces para cualquier $\tau^{\times}>0$, el control (4.19) satisface $\left\|u^{\times}(x)\right\|_{S} \leq 1$ y el sistema a lazo cerrado (4.1)-(4.19) es EGA.

Prueba. Como $\tau(x)$ es una solución del problema de optimización, entonces $\left\|u^{\times}(x)\right\|_{S} \leq 1$. Por otra parte, tomando en consideración a la hipótesis $\mathrm{H} 1$, que $\tau(x)$ es admisible, y el sistema a lazo cerrado (4.1)-(4.19), para toda $x \in \mathrm{R}^{n}$, obtenemos que

$$
\dot{V}(x)=\left\{\begin{array}{ll}
-\frac{1}{2} x^{\top} Q x+x^{\top} P B u_{\tau}(x), & \text { si } x \in \mathbb{R}^{n} \backslash \mathcal{E}\left(\tau^{\times}\right) \\
-\frac{1}{2} x^{\top} Q x-\frac{1}{\tau^{\times}}\left(x^{\top} P B B^{\top} P x\right), & \text { si } x \in \mathcal{E}\left(\tau^{\times}\right)
\end{array},\right.
$$

es semidefinida negativa. Luego, el punto de equilibrio $x=0$ del sistema a lazo cerrado es estable. Finalmente, la EGA del sistema (4.1)-(4.19) se sigue de la Proposición 2.1. 
Con respecto a una clase de incertidumbres que preservan la estabilidad de Lyapunov podemos hallar estabilidad robusta del sistema a lazo cerrado. Es decir, si

$$
\dot{x}=(A+\Delta A) x+B u
$$

es el sistema real, y el control (4.19) está diseñado con base en la matriz nominal $A$, el siguiente resultado es una consecuencia de la prueba de la Proposición 3.2.

Corolario 3.3. Supongamos que la matriz $A$ es robustamente Lyapunov estable con respecto a la incertidumbre $\triangle A$ (i.e., $(A+\Delta A)^{\top} P+P(A+\Delta A)=-Q_{\Delta}$, para alguna $Q_{\Delta} \geq 0$ ). Bajo las hipótesis de la Proposición 3.2, el control (4.19) satisface $\left\|u^{\times}(x)\right\|_{S} \leq 1$ y el sistema (4.21)-(4.19) es EGA.

El resultado anterior establece que el sistema a lazo cerrado es robustamente estable en la medida que la incertidumbre $\Delta A$ preserve la propiedad de estabilidad de Lyapunov. La preservación de la estabilidad de Lyapunov bajo perturbaciones suele hallarse en muchos sistemas físicos.

Con el objeto de satisfacer la restricción del control, $\|u(x)\|_{S} \leq 1$, el método de los multiplicadores de Lagrange [40] proporciona una condición necesaria y suficiente para que se tenga un extremo, condición que se reduce básicamente en hallar una función $c=c(\tau)$ como la solución de la relación (frontera elipsoidal)

$$
\partial \mathcal{E}(\tau)=\left\{x \in \mathbb{R}^{n}: V(x)=c(\tau)\right\},
$$

sujeta a la restricción $\|u(x)\|_{S} \leq 1$. Resolviendo tal problema de optimización, el hasta ahora parámetro $\tau$ se convierte en una función de los estados $(\tau=\tau(x))$. Este procedimiento será desarrollado en la siguiente sección.

\subsection{Resultado de estabilización global}

Consideremos el sistema lineal (4.1), donde $A$ es una matriz $n \times n$-dimensional que satisface la hipótesis $\mathrm{H} 1$ y $B$ es una matriz $n \times m$. De la Proposición 2.1, si además se satisface la hipótesis H2, la EGA del sistema (4.1) se garantiza mediante el control lineal (no acotado)

$$
u_{L}(x)=-\frac{1}{\tau} B^{\top} P x
$$

para $\tau>0$ fija.

Si el conjunto $\mathcal{B}=\left\{u \in \mathbb{R}^{m}:\left|u_{i}\right| \leq r_{i}, r_{i}>0, i=1, \ldots, m\right\}$ (una caja $m$-dimensional) se tomara como la restricción sobre el control, tendríamos que resolver un problema de programación c-parametrizado no suave. Por lo tanto, a fin de obtener una aproximación suave cuadrática de $\mathcal{B}$, se introduce aquí un elipsoide. Denotemos con $\|\cdot\|_{S}$ a la norma $S$-euclideana en $\mathbb{R}^{m}:\|u\|_{S}:=\sqrt{u^{\top} S u}$, donde $S:=R^{-2}$ y $R$ es una matriz $m \times m$ definida positiva y simétrica.

Por consiguiente, si suponemos que el control toma sus valores en la bola unitaria en la norma $S$-euclideana, $u \in \mathcal{B}_{1}^{2}=\left\{u \in \mathbb{R}^{m}:\|u(x)\|_{S} \leq 1\right\}$ (el elipsoide mencionado anteriormente), tenemos el siguiente resultado sobre entradas globalmente sujetas tanto a cotas sobre sus magnitudes como en sus derivadas. 
Teorema 4.1. Supongamos que el sistema lineal (4.1) satisface las hipótesis H1 y H2, y además, sin pérdida de generalidad, que $B$ tiene rango máximo. Entonces, la EGA del sistema lineal (4.1) se obtiene mediante la función $\mathrm{RCA} u^{\times}(x)$ dada en (4.19), que satisface $u^{\times} \in \mathcal{B}_{1}^{2}$, con

$$
u_{\tau}(x)=-\sqrt{\frac{2}{\lambda^{*} x^{\top} P x}} B^{\top} P x,
$$

donde $\tau^{*}>0$ es arbitrario, $y \lambda^{*}=\max \arg \left(\operatorname{det}\left(2 S B^{\top} P B-\lambda I\right)=0\right)$. Además, para toda $x \in \mathbb{R}^{n}$ tenemos que $\left\|d u^{\times} / d t\right\|_{S} \leq \kappa$, clonde $\kappa>0$ está definido en términos de las matrices $A, B$ y $P$, la matriz para cota en el control $S$ y el parámetro $\tau^{\times}$.

Prueba. Primero resolveremos la ecuación (4.22). Como el problema de programación (4.17) es convexo y para toda $x \neq 0, \nabla_{x} V(x) \neq 0$, la condición necesaria para un extremo es también suficiente: $x^{*}$ es una solución óptima del problema de programación (4.17) ssi existe una $\lambda^{*}>0$ tal que el par óptimo $\left(x^{*}, \lambda^{*}\right) \in \mathbb{R}^{n} \times \mathbb{R}$ satisface

$$
\nabla_{x}\left\|-B^{\top} P x^{*}\right\|_{S}^{2}-\lambda^{*} x^{* \top} P=0,
$$

donde $\nabla_{x}(\cdot)$ denota el gradiente respecto a $x$. Dado que $B$ tiene rango máximo, de (4.25) obtenemos

$$
\left(x^{* \top} P B\right)\left(2 S B^{\top} P B-\lambda^{*} I\right)=0,
$$

donde $I$ es la matriz identidad $m \times m$ y $\lambda^{*} \in \mathbb{R}$ (constante) es la mayor raíz positiva del polinomio $p(\lambda)=\operatorname{det}\left(2 S B^{\top} P B-\lambda I\right)$. También, de $(4.25)$, si $\left(x^{*}, \lambda^{*}\right)$ es una pareja óptima, para toda $x \in \partial \mathcal{E}\left(c^{*}\right)$, tenemos

$$
2 \tau^{2}\left(x^{*}\right)=2 x^{* \top} B P S B^{\top} P x^{*}=\lambda^{*} x^{* \top} P x^{*}=2 \lambda^{*} c\left(\tau\left(x^{*}\right)\right)=\lambda^{*} x^{\top} P x
$$

Por lo tanto,

$$
c(\tau)=\frac{\tau^{2}}{\lambda^{*}} \quad y \quad \tau(x)=\sqrt{\frac{\lambda^{*}}{2} x^{\top} P x} \quad \text { (una hipersuperficie cónica) }
$$

Entonces, de (4.18) con $\tau(x)$ dado en (4.28), obtenemos (4.24). Esto completa el problema de optimización. Por otra parte, la EGA del sistema a lazo cerrado (4.1)-(4.19) se garantiza con base en la Proposition 3.1. Finalmente, procederemos con la cota global sobre la derivada del control. Denotemos $\alpha=\sqrt{2 / \lambda^{*}}$, luego $\tau(x)=\alpha^{-1}\|x\|_{\rho}$. Así, $u_{\tau}(x)=-\alpha\|x\|_{P}^{-1} B^{\top} P x$. Entonces, del sistema a lazo cerrado (4.1)-(4.19) y retomando (4.2), obtenemos

$$
\begin{aligned}
u_{\tau} & =\frac{1}{\tau^{2}} \nabla \tau \dot{x} B^{\top} P x-\frac{1}{\tau} B^{\top} P \dot{x}=\frac{1}{\tau}\left(\frac{1}{\tau} \frac{1}{\alpha\|x\| \rho}\left(x^{\top} P \dot{x}\right) B^{\top} P x-B^{\top} P \dot{x}\right) \\
& =\frac{1}{\tau}\left(\frac{1}{(\alpha \tau)^{2}}\left(x^{\top} P A x+x^{\top} P B u_{\tau}\right) B^{\top} P x-B^{\top} P A x-\left(B^{\top} P B\right) u_{\tau}\right) \\
& =\frac{1}{\tau}\left(\left(\frac{1}{2 \alpha^{2} \tau}\left(x^{\top} Q x\right)+\frac{1}{\alpha^{2}}\left(u_{\tau}^{\top} u_{\tau}\right)\right) u_{\tau}-\left(B^{\top} P B\right) u_{\tau}-B^{\top} P A x\right) .
\end{aligned}
$$

Si $\|\cdot\|$ denota la 2 -norma matricial inducida, entonces $\lambda^{*}=2\left\|S B^{\top} P B\right\|$, y obtenemos

$$
\left\|i_{\tau}\right\|_{S} \leq \frac{1}{\tau}((\underbrace{\frac{1}{2 \alpha^{2} \tau}\left\|Q^{1 / 2} x\right\|_{I}^{2}}_{(a)}+\underbrace{\frac{1}{\alpha^{2}}\left\|u_{\tau}\right\|_{I}^{2}}_{(b)})\left\|u_{\tau}\right\|_{S}+\underbrace{\left\|B^{\top} P B u_{\tau}\right\|_{S}}_{(c)}+\underbrace{\left\|B^{\top} P A x\right\|_{S}}_{(d)})
$$

Derivemos a continuación otras cotas más útiles para las expresiones (a), (b), (c) y (d): 
(a) $\left\|Q^{1 / 2} x\right\|_{I}^{2} \leq\left\|Q^{1 / 2} P^{-1 / 2}\right\|^{2}\|x\|_{P}^{2}$

(b) $\left\|u_{\tau}\right\|_{I}^{2} \leq \lambda_{\min }^{-2}\left(S^{1 / 2}\right)=\lambda_{\max }^{2}\left(S^{-1 / 2}\right)=\left\|S^{-1 / 2}\right\|^{2}$;

(c) $\left\|B^{\top} P B u_{\tau}\right\|_{S}=\left\|S^{1 / 2} B^{\top} P B S^{-1 / 2} S^{1 / 2} u_{\tau}\right\|_{I} \leq\left\|S^{1 / 2} B^{\top} P B S^{-1 / 2}\right\|\left\|u_{\tau}\right\|_{S} ;$

(d) $\left\|B^{\top} P A x\right\|_{S}=\left\|\left(S^{1 / 2} B^{\top} P A P^{-1 / 2}\right) P^{1 / 2} x\right\|_{I} \leq\left\|S^{1 / 2} B^{\top} P A P^{-1 / 2}\right\|\|x\|_{P}$.

Entonces, reemplazando las cotas dadas en (4.29) con las previamente obtenidas, tenemos

$$
\begin{aligned}
\left\|i_{\tau}\right\|_{S} \leq & \left(\frac{1}{2 \alpha^{2} \tau}\left\|Q^{1 / 2} P^{-1 / 2}\right\|^{2}\|x\|_{P}^{2}+\frac{1}{\tau}\left(\frac{1}{\alpha^{2}}\left\|S^{-1 / 2}\right\|^{2}+\left\|S^{1 / 2} B^{\top} P B S^{-1 / 2}\right\|_{1}\right)\left\|u_{\tau}\right\|_{S}\right. \\
& +\frac{1}{\tau}\left\|S^{1 / 2} B^{\top} P A P^{-1 / 2}\right\|\|x\|_{P} .
\end{aligned}
$$

Consecuentemente, de la definición de $\tau(x)$, del hecho de que ésta es una función monótona creciente y como $\left\|u_{\tau}\right\|_{S} \leq 1$, finalmente obtenemos, para toda $x \in \mathbb{R}^{n}$

$$
\begin{aligned}
\left\|u^{\times}\right\|_{S} \leq & \frac{1}{2}\left\|Q^{1 / 2} P^{-1 / 2}\right\|^{2}+\frac{1}{\tau^{\times}}\left(\frac{1}{\alpha^{2}}\left\|S^{-1 / 2}\right\|^{2}+\left\|S^{1 / 2} B^{\top} P B S^{-1 / 2}\right\|\right) \\
& +\frac{1}{\alpha}\left\|S^{1 / 2} B^{\top} P A P^{-1 / 2}\right\|,
\end{aligned}
$$

donde, $\kappa$ se define como la parte derecha de la desigualdad anterior.

Corolario 4.2. Supongamos que el sistema lineal con control escalar (4.1) satisface las hipóteses $\mathrm{H} 1$ y H2. Entonces, la EGA del sistema lineal se obtiene mediante la función $\mathrm{RCA} u^{\times}(x)$ dada en (4.19), que satisface $\left|u^{\times}\right| \leq r, r>0$, con

$$
u_{\tau}(x)=-\frac{r}{\|b\|_{P}\|x\|_{P}} b^{\top} P x
$$

donde $\tau^{\times}>0$ es arbitrario. Además, para toda $x \in \mathbb{R}^{n}$, tenemos

$$
\left|u^{\times}\right| \leq \kappa:=r\left(\frac{1}{2}\left\|Q^{1 / 2} P^{-1 / 2}\right\|^{2}+\frac{2 \sqrt{3}}{9 \tau^{\times}}\|b\|_{P}^{2}+\left\|P^{1 / 2} A P^{-1 / 2}\right\|\right) .
$$

Prueba. Como $B=b \in \mathbb{R}^{n}$, entonces $S:=r^{-2}>0$. De aquí que, a partir de (4.26) tenemos que $b^{\top} P x^{*} \neq 0$, donde $x^{*}$ es una solución óptima. Ahora, si consideramos (4.28), entonces

$$
c(\tau)=\frac{\tau^{2} r^{2}}{2 b^{\top} P b} \quad \text { y así } \quad \tau(x)=\frac{1}{r}\|b\|_{P}\|x\|_{P}
$$

Por lo tanto, de (4.18) con $\tau(x)$ dada en (4.33), obtenemos (4.31). Además, la cota sobre la derivada admite una expresión más simple que (4.30). En efecto,

$$
\begin{aligned}
& u_{\tau}=\frac{1}{2 \alpha^{2} \tau^{2}}\left(x^{\top} Q x\right) u_{\tau}+\frac{1}{\tau}\left(\left(\frac{1}{\alpha^{2}} u_{\tau}^{2}-\left(b^{\top} P b\right)\right) u_{\tau}-b^{\top} P A x\right) . \text { Entonces, } \\
& \left|u_{\tau}\right| \leq \frac{\left(b^{\top} P b\right)}{2 r^{2} \tau^{2}}\left(x^{\top} Q x\right)\left|u_{\tau}\right|+\frac{1}{\tau}\left(\left(1-\frac{1}{r^{2}} u_{\tau}^{2}\right)\left(b^{\top} P b\right)\left|u_{\tau}\right|+\left|b^{\top} P A x\right|\right) .
\end{aligned}
$$


De lo cual, y trabajando de manera análoga al derivar (4.30), finalmente obtenemos (4.32).

Observación 3. Basados en la hipótesis H2, tenemos que $\tau(x)$ dada en (4.28) es una función de Lyapunor propia (i.e., es definida positiva, radialmente no acotada $y d \tau / d t<0$ para toda $\left.x \in \mathbb{R}^{n} \backslash\{0\}\right)$, y es una función no Lipschitsz en el origen.

Observación 4. La cota $\kappa$ admite una interpretación geométrica en términos de la curvatura del elipsoide $\mathcal{E}(\tau)$ y el conjunto elipsoidal de controles $\mathcal{B}_{1}^{2}$. En efecto, observemos que

$$
\left\|P^{1 / 2} A P^{-1 / 2}\right\| \leq\|A\|\left\|P^{1 / 2}\right\|\left\|P^{-1 / 2}\right\| \leq\|A\|\left(\frac{\zeta_{\max }}{\zeta_{\min }}\right)
$$

donde $\zeta_{\min }^{-1}=\lambda_{\max }\left(P^{1 / 2}\right)=\left\|P^{1 / 2}\right\|$ y $\zeta_{\max }=\lambda_{\max }\left(P^{-1 / 2}\right)=\left\|P^{-1 / 2}\right\|$, corresponden a la minima y la máxima longitudes de los semiejes de $\left.\partial \mathcal{E}(\tau)\right|_{c=1 / 2}$. Un resultado análogo puede obtenerse en términos de $S$. De esta forma, en la medida que ambos elipsoides se aproximen a una bola, el valor de $r$ se verá minimizado.

Observación 5. Podemos dar una conexión con un resultado dado en [63]. En ese trabajo, se obtuvo la EGA mediante una retroalimentación lineal saturada para el caso simple de sistemas lineales (4.1) tales que los eigenvalores de la matriz $A$ son simples con parte real cero. Entonces existe un transformación de semejanza $T$ tal que $A^{\prime}=T^{-1} A T$ es antisimétrica. Por lo tanto, identificando $A$ con $A^{\prime}$, obtenemos que $P=p I$ (donde $p>0$ e $I$ es la matriz identidad $n \times n$ ), satisface (4.2), y el sistema lineal es EGA mediante la retroalimentación

$$
u_{\tau}(x)=-\frac{r}{\sqrt{\left\|B^{\top} B\right\|}\|x\|} B^{\top} x
$$

que satisface $\left\|u_{\tau}(x)\right\|_{I} \leq r$. Observenos que $u_{\tau}(x)$ (4.35) no depende de la elección de la matriz $P$. Así, este control es óptimo en el sentido de la cota sobre su derivada: $\left\|d u^{\times} / d t\right\|_{I} \leq r \kappa=$ $r\left(\|A\|+2\left\|B^{\top} B\right\| / \mu^{\times}\right)$-observemos que $\kappa$ es $P$-independiente, pues $\tau^{\times}=p \mu^{\times}$.

Observación 6. Otra reformulación de la función de control $u(x)$, que también evita la singularidad en 0 , está dada por la siguiente función RCA global asintótica. Definamos la función de control $(\tau, \hat{\epsilon})$-dependiente

$$
u_{\varepsilon}(x):=-\frac{1}{\varepsilon+\tau(x)} B^{\top} P x
$$

donde $\varepsilon>0$, es un parámetro de sintonía suficienientente pequeño. Es fácil probar que $u_{\varepsilon}(x)$ estabiliza global y asintóticamente al sistema (4.1). Observemos que el control (4.36) nunca alcanza sus extremos, $\left\|u_{\varepsilon}(x)\right\|_{S}<1$; lo cual significa que el control (4.36) no usia todo el recurso de control disponible. Por otra parte, una combinación de este control con (4.19) permite que $\kappa$ dependa también de $\varepsilon$; lo cual da lugar, aunque sólo semiglobalmente, a un mayor rango de cotas sobre la derivada del control.

Observación 7. Vale la pena mencionar que el control (4.24) es una aproximación suave al control óptimo (discontinuo) construido en [8], para el caso de sistemas lineales autónomos. En ese trabajo, si $x \notin \Sigma=\left\{x \in \mathbb{R}^{n}: B^{\top} P x=0\right\}$, el control óptimo no singular $u_{o p}(x):=$ $-\left(B^{\top} P x\right) /\left\|B^{\top} P x\right\|_{S}$ se obtiene resolviendo cierto índice cuadrático; mientras que, cuando $x \in$ $\Sigma$, los fenómenos singulares resultantes son estudiados en detalle. 


\subsection{Estabilización semiglobal para los sistemas lineales ACOCA}

Debido a que en muchos problemas prácticos uno está interesado en regiones acotadas, se justifica obtener una propiedad de estabilizabilidad semiglobal asintótica en lugar de la global. Una desventaja principal del enfoque semiglobal radica en que al tener una región de estabilidad grande implica bajas ganancias y en consecuencia, una convergencia lenta, cf. [2]-[37]-[48]. En esta sección, abordaremos este problema aplicando el esquema de ganancias programadas desarrollado en la sección anterior y las ideas geométricas consideradas en el Capítulo 3 (o [60]).

Un resultado semiglobal para los sistemas lineales ACOCA puede obtenerse como sigue. Consideremos un conjunto acotado arbitrario $D \subset \mathbb{R}^{n}$. Sea $\Pi(\theta)>0$ una solución de la siguiente ecuación algebraica de Riccati (EAR)

$$
A^{\top} \Pi+\Pi A-2 \Pi B B^{\top} \Pi+\frac{1}{\theta} Q=0,
$$

donde $\theta>0$, y $Q$ es una matriz simétrica definida positiva [60]. Definamos la función cuadrática

$$
V(x)=\frac{1}{2} x^{\top} \Pi x
$$

Con base en lo desarrollado en el Capítulo 3, supongamos que la entrada $u$ toma sus valores en el conjunto compacto $\mathcal{B}_{r}^{\infty}=\left\{u \in \mathbb{R}^{n}:\left|u_{i}\right| \leq r, i=1, \ldots, m\right\}$, la bola de radio $r>0$ en la $\infty$-norma en $\mathbb{R}^{m}$ (un $r$-hipercubo). Entonces, existe $\theta>0$ tal que $D \subset \mathcal{E}_{\theta}$, donde

$$
\mathcal{E}_{\theta}=\left\{x \in \mathbb{R}^{n}: V(x) \leq c_{\theta}^{*}\right\},
$$

para algún $c_{\theta}^{*}$, especificado en $(4.41)$ y además, la retroalimentación

$$
u_{\theta}(x)=-B^{\top} \Pi x
$$

satisface $\left|u_{\theta_{i}}(x)\right| \leq r, i=1, \ldots, m$, para toda $x \in \mathcal{E}_{\theta}$. El procedimiento de optimización desarrollado en la sección anterior no puede ser aplicado aquí porque $\theta$ está implícitamente definida en términos de la matriz $\Pi$ (y viceversa). Consecuentemente, tomemos una $\theta^{*}$ fija, y transformemos el problema con restricciones en $u_{\theta}(x)$ en el problema de estimar los valores mínimo y máximo de $u_{\theta_{i}}$ en la hipersuperficie $\partial \mathcal{E}_{\theta}=\left\{x \in \mathbb{R}^{n}: \theta(x)=\theta^{*}\right\}$. Luego, el parámetro asociado $c_{\theta}^{*}$ se define por

$$
c_{\theta}^{*}=\min \left\{c_{1 \theta}, \ldots, c_{m \theta}\right\}, \quad c_{\theta_{i}}=\frac{r^{2}}{2 b_{i}^{\top} \Pi\left(\theta^{*}\right) b_{i}} .
$$

Definamos $N x:=A x+B u_{\theta}(x)=\left(A-B B^{\top} \Pi\right) x$, tal que su espectro se localice en el semiplano izquierdo abierto del plano complejo, $\sigma(N) \subset \mathbb{C}^{-}$. Entonces en la región $\mathcal{E}_{\theta}$, tenemos que $\left|u_{\theta_{i}}(x)\right| \leq r, i=1, \ldots, m, \dot{x}=N x$, y $x(t) \rightarrow 0$, cuando $t \rightarrow \infty$ (asintóticamente). Es decir, el conjunto $D$ está contenido en la región de atracción del origen.

Sin embargo, la retroalimentación $u_{\theta}(x)$ es del tipo de baja ganancia: si la región $\mathcal{E}_{\theta}$ es grande, las ganancias son proporcionalmente pequeñas. Por lo tanto, redefiniremos el control $u_{\theta}(x)$ de forma tal que obtengamos un control de alta ganancia en la región $\mathcal{E}_{\theta}$. La idea es diseñar un control $u(x)$ cuyas ganancias sean funciones monótonas decrecientes en $\tau$ y tales que 
si $\tau \rightarrow x$. entonces $u(x)=u_{\theta}(x)$-el control de baja ganancia sea recobrado en $\partial \mathcal{E}_{\theta}$. El control propuesto es del tipo de baja ganancia $(\theta)$, alta ganancia $(\tau)[48]$,

$$
u_{\tau}(x)=\left(1+\frac{1}{\tau}\right) u_{\theta}(x), \tau>0
$$

Entonces, debemos resolver un problema de optimización análogo a (4.16), dado por

$$
\min _{x} \tau \geq 0, \quad \text { t.q. } \quad\left(1+\frac{1}{\tau}\right)\left\|u_{\theta}(x)\right\|_{I} \leq r \quad \text { y } \quad c_{\theta}(\tau)=V(x)=\frac{1}{2} x^{\top} \Pi x .
$$

Teorema 5.1. Supongamos que el sistema lineal (4.1) es ACOCA, y que además, sin pérdida de generalidad, $B$ tiene rango máximo. Consideremos un conjunto acotado $D \subset \mathbb{R}^{n}$ y sea $\Pi(\theta)>0$ una solución de EAR (4.3T), donde $\theta$ se elige de manera tal que $D \subset \mathcal{E}_{\theta}(4.39)$. Redefinamos la función de control $u^{\times}(x)$ dada en (4.19) reemplazando $\tau^{\times}$con $\gamma^{\times}$. Entonces. para cualuier $0<\gamma^{x} \leq 1$, el control modificado $u^{x}(x)$ con

$$
u_{\tau}(x)=-\frac{1}{\gamma(x)} B^{\top} \Pi x
$$

es una función RCA que satisface $u^{\times} \in \mathcal{B}_{r}^{2}=\left\{u \in \mathbb{R}^{m}:\|u\|_{I} \leq r\right\}$-la bola euclideana de radio $r, y$ con base en la cual el sistema a lazo cerrado resultante es semiglobal asintóticamente estable en la región $\mathcal{E}_{\theta}$, donde $\gamma(x):=\alpha^{-1}\|x\|_{\Pi}$, con $\alpha=r / \beta^{*} y \beta^{*}=\left(\max _{i} b_{i}^{\top} \Pi b_{i}\right)^{1 / 2}$. Además, para toda $x \in \mathcal{E}_{\theta}$, tenemos $\left\|d u^{\times} / d t\right\|_{I} \leq k$, donde $n>0$ está definido en téminos de las matrices $A, B y$. II, la cota del control $r y$ el parámetro $\gamma^{x}$.

Prueba. La demostración es similar a la prueba del Teorema 4.1. Supongamos cue $\Pi=$ $\Pi(\theta)>0$ es una solución de la $\operatorname{EAR}(4.37)$, y consideremos una versión adecuada de (4.25). Debido a la convexidad del programa (4.43) y como para toda $x \neq 0, \nabla_{x} V(x) \neq 0$, la condición necesaria para un extremo es también suficiente. Entonces, después de algunas manipulaciones algebraicas, obtenemos

$$
c_{\theta}(\tau)=\frac{c_{\theta}^{*}}{\left(1+\frac{1}{\tau}\right)^{2}}=c_{\theta}^{*} \gamma^{2},
$$

donde $c_{\theta}^{*}$ esté dado en (4.41). Por lo tanto,

$$
\partial \mathcal{E}_{\theta}(\tau)=\left\{x \in \mathbb{R}^{n}: V(x)=\frac{1}{2} x^{\top} \Pi \amalg=c_{\theta}(\tau)\right\} .
$$

De lo cual,

$$
\gamma(x)=\frac{\beta^{*}}{r} \sqrt{x^{\top} 11 x} \quad y \quad \tau(x)=\frac{\beta^{*} \sqrt{x^{\top} \mathrm{II} x}}{r-\beta^{*} \sqrt{x^{\top} \mathrm{II} x}} .
$$

Así, de (4.42) con $\gamma(x)$ dado en (4.47), obtenemos (4.44). Esto completa el problema de optimización. Es fácil verificar que $\tau(x)$ es admisible en $\mathcal{E}_{0}$ : es ma función suave (excepto en $x=0$ ) definida positiva en $\mathcal{E}_{\theta} y$, para cualquier $\tau^{\times} \geq 0$, se satisface la igualdad $\partial \mathcal{E}_{0}\left(\tau^{\times}\right)=$ $\partial S_{\tau^{\times}}=\left\{x \in \mathbb{R}^{n}: \tau(x)=\tau^{\times}\right\}$.

Consecuentemente, en vista que $\gamma(x)$ es una función monótona creciento, tal que en $\mathcal{E}_{b}$, $0 \leq \gamma(x) \leq 1, \operatorname{con} \gamma(x){ }_{b \varepsilon_{\theta}} \equiv 1$, la propiedad de estabilidad semiglobal se obtienc observando que dentro de $\mathcal{E}_{0} \backslash \mathcal{E}_{\theta}\left(\tau^{\times}\right)$.

$$
\dot{V}(x)=-x^{\top}\left(\frac{1}{20} Q+\left(\frac{1}{\gamma(x)}-1\right) \operatorname{\Pi I} B B^{\top} \Pi\right) x
$$


es definida negativa. Finalmente, procederemos con la cota semiglobal sobre la derivada de la entrada. Retomando (4.37), $u_{\tau}(x)(4.44)$ y algunos cálculos algebraicos, obtenemos

$$
\begin{aligned}
\dot{u}_{\tau} & =\frac{1}{\alpha^{2} \gamma^{2}}\left(x^{\top} \Pi A x+x^{\top} \Pi B u_{\tau}\right) \frac{1}{\gamma} B^{\top} \Pi x-\frac{1}{\gamma}\left(\left(B^{\top} \Pi B\right) u_{\tau}+B^{\top} \Pi A x\right) \\
& =\frac{1}{\alpha^{2}}\left(\frac{1}{2 \theta \gamma^{2}} x^{\top} Q x+\left(\frac{1}{\gamma}-1\right)\left\|u_{\tau}\right\|_{I}^{2}\right) u_{\tau}-\frac{1}{\gamma}\left(\left(B^{\top} \Pi B\right) u_{\tau}+B^{\top} \Pi A x\right) .
\end{aligned}
$$

De lo cual, y como $\left\|u_{\tau}\right\|_{I} \leq r$, mediante una derivación análoga a (4.30), obtenemos finalmente, para toda $x \in \mathcal{E}_{\theta}$,

$$
\left\|u^{x}\right\|_{I} \leq r\left(\frac{1}{2 \theta}\left\|Q^{1 / 2} \Pi^{-1 / 2}\right\|^{2}+\left(\frac{1}{\gamma^{x}}-1\right) \beta^{* 2}+\frac{1}{\gamma^{x}}\left\|B^{\top} \Pi B\right\|+\frac{1}{\beta^{*}}\left\|B^{\top} \Pi A \Pi^{-1 / 2}\right\|\right)
$$

donde, $\kappa$ se define como la parte derecha de la desigualdad anterior.

Observación 8. Debemos remarcar que un control similar a (4.42) fue también propuesto en [48]. Sin embargo, en ese artículo, $\rho(:=1 / \tau)$ era considerado como un parámetro de sintonía; mientras que en este capítulo, se introduce como una solución de un procedimiento de optimización con lo cual $\tau=\tau(x)$-una función de los estados. De esta forma, el problema de programación (4.43) puede ser concebido como un procedimiento para sintonizar el parámetro de alta ganancia $\tau$ (de hecho, un sintonizador dependiente de los estados).

\subsection{Ejemplos}

Ejemplo 6.1. Consideremos las siguientes ecuaciones normalizadas de moviniento para un navegador inercial [9]

$$
\left(\begin{array}{l}
\dot{x}_{1} \\
\dot{x}_{2} \\
\dot{x}_{3}
\end{array}\right)=\left(\begin{array}{ccc}
0 & -1 & 0 \\
1 & 0 & 1 \\
0 & 0 & 0
\end{array}\right)\left(\begin{array}{l}
x_{1} \\
x_{2} \\
x_{3}
\end{array}\right)+u\left(\begin{array}{l}
0 \\
0 \\
1
\end{array}\right), \quad|u| \leq r
$$

donde $x_{1}$ es el error de la velocidad hacia el Este, $x_{2}$ es la inclinación de la plataforma respecto al eje Norte, $x_{3}$ es la desviación del giroscopio norte y la entrada $u$ es la desviación de la razón de cambio del giroscopio. Para el sistema no controlado ((4.50) con $u \equiv 0)$, el origen es un punto de equilibrio marginalmente estable: $\sigma(A)=\{0, \pm i\}$. Así, la hipótesis $\mathrm{H} 1$ se satisface, y unas matrices $P$ and $Q$ que satisfacen la ecuación (4.2) son, e.g.

$$
P=\left(\begin{array}{ccc}
p & 0 & p \\
0 & p & 0 \\
p & 0 & q
\end{array}\right)>0 \quad \text { y } Q=0=03 \times 3,
$$

donde $p>0$ y $q-p>0$. Además, un cálculo fácil muestra la validez de la hipótesis H2, lo cual garantiza la EGA del sistema a lazo cerrado dado por (4.50) y la retroalimentación (no acotada)

$$
u_{u n b}(x)=-b^{\top} P x=-\left(p x_{1}+q x_{3}\right) .
$$


No es difícil de probar que una retroalimentación lineal saturada (RLS) simple basada en (4.52) da lugar también a la EGA del sistema. Fijemos la condición inicial $\bar{x}=(1,-3,4)$, la cota de la entrada en $r=1$ y las entradas de la matriz $P: p=2$ y $q=3$. Las evoluciones de los estados del sistema a lazo cemado correspondiente se muestran en la Figura 1 (a). Como se puede ver en la Figura 1 (b), esta RLS se comporta como un control de tipo discontinuo, pues tiene variaciones muy rápidas. En el caso en que esta característica fuera indeseable, el método de diseño propuesto puede aplicarse para salvar este "mal funcionamiento". De (4.33), tenemos la ley de control acotada

$$
u_{\tau}(x)=\frac{-r\left(p x_{1}+q x_{3}\right)}{\sqrt{p q\left(x_{1}^{2}+x_{2}^{2}+2 x_{1} x_{3}\right)+q^{2} x_{3}^{2}}} .
$$

Usamos una versión rediseñada de la $\mathrm{RCA}$ (4.53) basada en (4.19) con $\tau^{\times}=1$, de manera que el sistema a lazo cerrado resultante deviene EGA. En la Figura 2 (a), puede observarse que la función rediseñada RCA da una dinámica satisfactoria del sistema a lazo cerrado (4.50)-(4.53). En la Figura 2(b), se muestra cómo este control tiene un comportamiento mejorado sobre el de la RLS. Finalmente, la cota global sobre la derivada de la entrada $r$ dada en (4.32), que corresponde a $p=2, q=3, r=1$ y $\tau^{\mathrm{x}}=1$, es $\kappa=1+2 \sqrt{3} / 3 \approx 2.1547$; mientras que usando un programa de optimización, el máximo valor global que alcanza $\left|d u^{\times} / d t\right|$ es cercano a 1.83 .

Ejemplo 6.2. Consideremos el siguiente modelo no lineal de un puente de carga (crane with. hanging load o loading bridge) [1]-[9]

$$
\begin{gathered}
\left(\begin{array}{c}
\dot{x}_{1} \\
\dot{x}_{2} \\
\dot{x}_{3} \\
\dot{x}_{4}
\end{array}\right)=\left(\begin{array}{c}
x_{2} \\
f_{2}\left(x_{3}, x_{4}\right) \\
x_{4} \\
f_{4}\left(x_{3}, x_{4}\right)
\end{array}\right)+u\left(\begin{array}{c}
0 \\
\frac{1}{m_{c}+m_{L} \operatorname{sen}^{2} x_{3}} \\
0 \\
-\frac{\cos x_{3}}{L\left(m_{c}+m_{L} \operatorname{sen}^{2} x_{3}\right)}
\end{array}\right), \quad|u| \leq r, \\
\operatorname{con} \quad f_{2}\left(x_{3}, x_{4}\right)=\frac{\left(g \cos x_{3}+L x_{4}^{2}\right) m_{L} \operatorname{sen} x_{3}}{m_{c}+m_{L} \operatorname{sen}^{2} x_{3}} \\
f_{4}\left(x_{3}, x_{4}\right)=-\frac{\left(g+L x_{1}^{2} \cos x_{3}\right) m_{L} \operatorname{sen} x_{3}+g m_{c} \operatorname{sen} x_{3}}{L\left(m_{c}+m_{L} \operatorname{sen}^{2} x_{3}\right)},
\end{gathered}
$$

donde $x_{1}$ es la posición del "crab", $x_{2}$ es su velocidad $\left(\dot{x}_{1}=x_{2}\right), x_{3}$ es el ángulo del cable (en radianes), $x_{4}$ es la velocidad anguiar $\left(\dot{x}_{3}=x_{4}\right)$ y la entrada $u$ es la fuerza que acelera el "crab". Los parámetros considerados son la masa del "crab" $m_{c}>0$, la longitud del cable $L>0$, la masa de la carga $m_{L}>0$ y la aceleración de la gravedad $g=9.81 \mathrm{~m} / \mathrm{s}^{2}$. El modelo no lineal del puente de carga (4.54) se linealiza para un ángulo pequeño de deflexión $x_{3}$ y una velocidad angular pequeña $x_{4}$. Si hacernos $\cos x_{3} \approx 1$, sen $x_{3} \approx x_{3}, \operatorname{sen}^{2} x_{3} \approx 0$ y $x_{4}^{2} \approx 0$, obtenemos el siguiente modelo lineal

$$
\left(\begin{array}{l}
\dot{x}_{1} \\
\dot{x}_{2} \\
\dot{x}_{3} \\
\dot{x}_{4}
\end{array}\right)=\left(\begin{array}{llll}
0 & 1 & 0 & 0 \\
0 & 0 & a & 0 \\
0 & 0 & 0 & 1 \\
0 & 0 & b & 0
\end{array}\right)\left(\begin{array}{l}
x_{1} \\
x_{2} \\
x_{3} \\
x_{4}
\end{array}\right)+u\left(\begin{array}{l}
0 \\
c \\
0 \\
d
\end{array}\right), \quad|u| \leq r
$$


donde los parámetros de entrada de la matriz $\left(a, b, c\right.$ and $d$ ) se definen como: $c=1 / m_{c}$, $a=m_{L} g c, d=-c / L$ y $b=\left(m_{L}+m_{c}\right) g d$. En este caso, aunque el sistema a lazo abierto ((4.55) con $u \equiv 0)$ no es estable en el sentido de Lyapunov (la hipótesis H1 no se satisface), es un sistema lineal ACOCA: El origen es un punto de cquilibrio marginalmente inestable, $\sigma(A)=\{0,0, \pm i \sqrt{|b|}\}$. Además, las matrices II y $Q$ pueden elegirse de la ecuación (4.37), e.g. $\Pi(\theta)=\left(\pi_{i j}\right)>0$ and $Q=\operatorname{diag}\left(q_{1}, q_{2}, q_{3}, q_{4}\right)>0$. Así, el sistema a lazo cerrado dado por (4.55) y el control (no acotado) (4.40)

$$
u_{\theta}(x)=-b^{\top} \Pi x=-\left(k_{1} x_{1}+k_{2} x_{2}+k_{3} x_{3}+k_{4} x_{4}\right),
$$

es EGA, donde $k_{1}=c \pi_{12}+d \pi_{14}, k_{2}=c \pi_{22}+d \pi_{24}, k_{3}=c \pi_{32}+d \pi_{34}$ y $k_{4}=c \pi_{42}+d \pi_{44}$.

Con el objeto de resolver la EAR (4.37) con una matriz diagonal $Q$, se proponen las entradas $\pi_{23}:=\left(m_{L}+m_{c}\right) k_{3}$ y $\pi_{34}:=L m_{L} k_{3}$; de esta forma, la ganancia $k_{3}$ se reduce a $\sqrt{q_{3} / 2 \theta}$ y así las entradas restantes de la matriz. II pueden obtenerse en términos de las ganancias del control definidas por $k_{1}:=\sqrt{q_{1} / 2 \theta}, k_{2}:=\sqrt{\pi_{12}+q_{2} / 2 \theta}, k_{3}$ y $k_{4}:=\sqrt{\pi_{34}+q_{4} / 2 \theta}$ y el sistema no lineal de ecuaciones algebraicas resultado de (4.37). Para los propósitos de simulación, se tomó como condición inicial a $\bar{x}=(70,-0.1,0.003,0)$, los parámetros $m_{c}=200, m_{L}=1000, L=10 \mathrm{y}$ $\theta=1$, la cota del control $r=10$; la matriz $Q$ elegida es diag $(2,63,72,50)$, así las ganancias son $k_{1}=1, k_{2}=35.075, k_{3}=6 \mathrm{y} k_{4}=245$. Además, con el objeto de comparar las técnicas alternas de diseño de control, aplicamos los diseños de control al modelo original no lineal (4.54). En primer lugar, se puede usar una retroalimentación lineal saturada (RLS) simple basada en (4.56) para estabilizar el sistema. Las evoluciones de los estados del sistema a lazo cerrado correspondiente se muestran en la Figura 3(a), y la del control saturado en la Figura 3(b). Sin embargo, este control se comporta como uno discontinuo, pues tiene variaciones "muy rápidas", i.e. $|\dot{u}| \gg 1$. En este caso, esta característica es ciertamente indeseable, porque la dinámica y las no linealidades del motor de tracción se descartaron a fin de simplicar el modelo (4.54) [1]. Estos supuestos implican que el diseño del control debe garantizar que tanto $|u|$ como $|\dot{u}|$ no sean excesivamente grandes. Por lo tanto, el método de diseño propuesto es apropiadamente aplicado en este caso. De (4.44), tenemos la ley de control acotada

$$
u_{\tau}(x)=\frac{r u_{\theta}(x)}{\sqrt{\left(c^{2} \pi_{22}+2 c d \pi_{24}+d^{2} \pi_{44}\right)\left(x^{\top} \Pi x\right)}},
$$

donde $x^{\top} \mathrm{II} x=\sum_{i, j} x_{i} x_{j} \pi_{i j}$.

Aquí usamos una versión rediseñada de la RCA (4.57) basada en (4.36) con $\varepsilon=0.1$, de manera que $x=0$ es un punto de equilibrio asintóticamente stable. Como puede verse en la Figura 4 (a), esta RCA rediseñada da una dinámica satisfactoria del sistema a lazo cerrado (4.54)-(4.57); y en la Figura 4(b), podemos apreciar el desempeño de este control. 

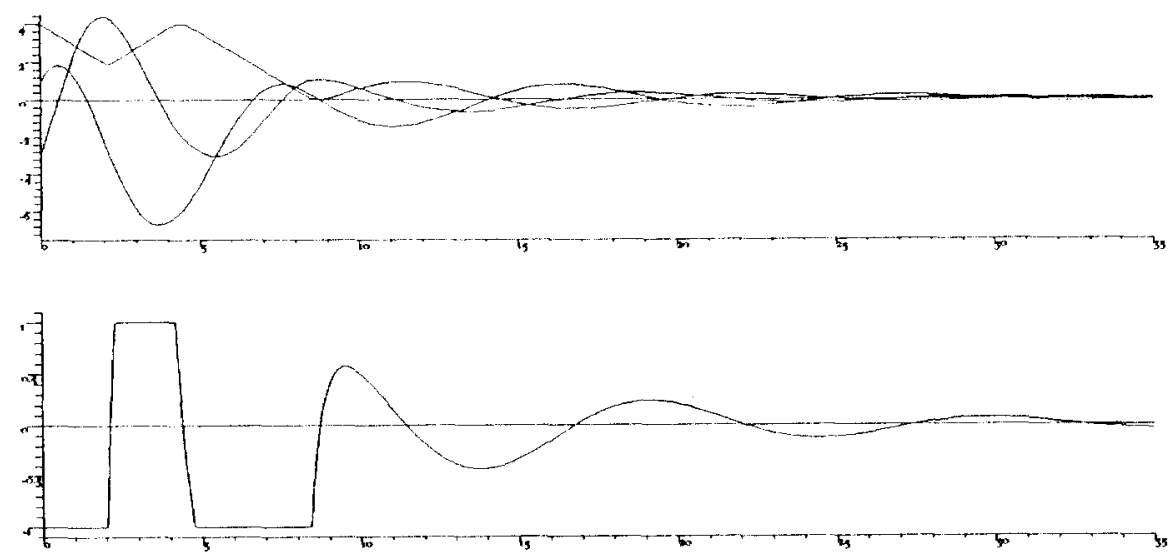

Figure 0.1: Ejemplo 6.1. Evolución de los estados del navegador inercial (4.50) correspondientes a una RLS basada en (4.52), arriba; y el desempeño del control, abajo; para la condición inicial $\bar{x}=(1,-3,4)$.
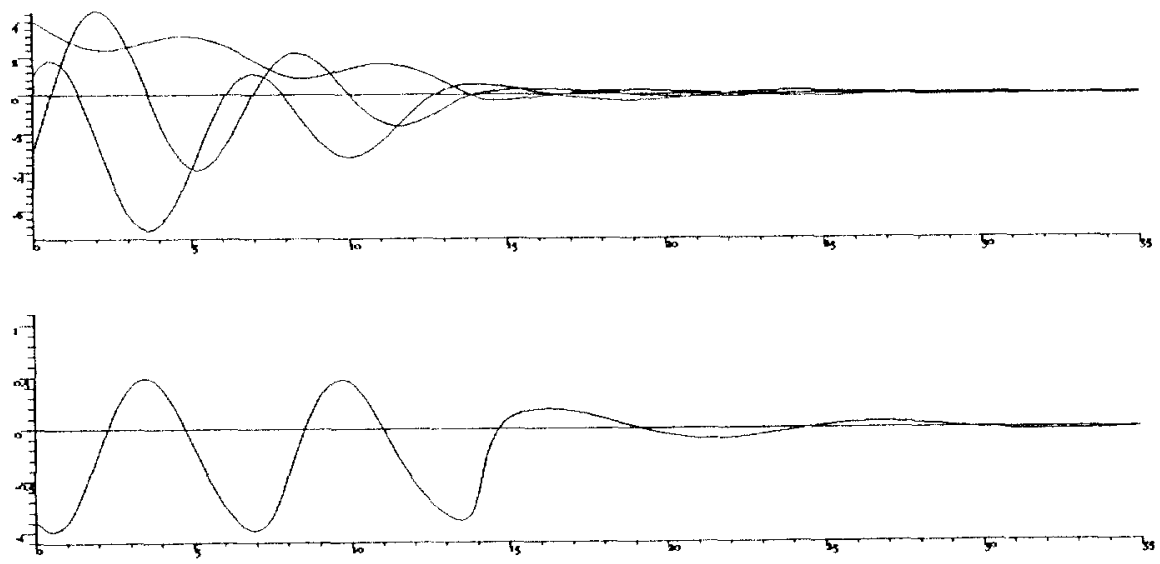

Figure 0.2: Ejemplo 6.1. Evolución de los estados del navegador inercial (4.50) correspondientes a la función $\mathbf{R C A}$ rediseñada (4.53) $\left(\tau^{\times}=1\right)$, arriba; y el desempeño del control, abajo; para la condición inicial $\bar{x}=(1,-3,4)$. 

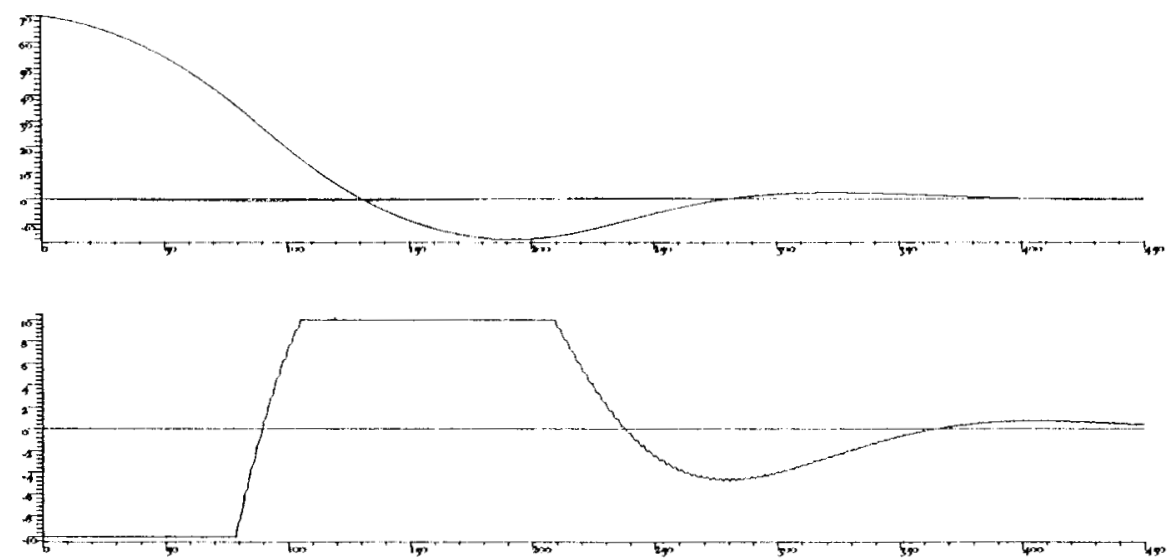

Figure 0.3: Ejemplo 6.2. Evolución de los estados del puente de carga (crane) (4.54) correspondientes a una RLS basada en (4.56), arriba; y el desempeño del control, abajo; para la condición inicial $\bar{x}=(70,-0.1,0.003,0)$.
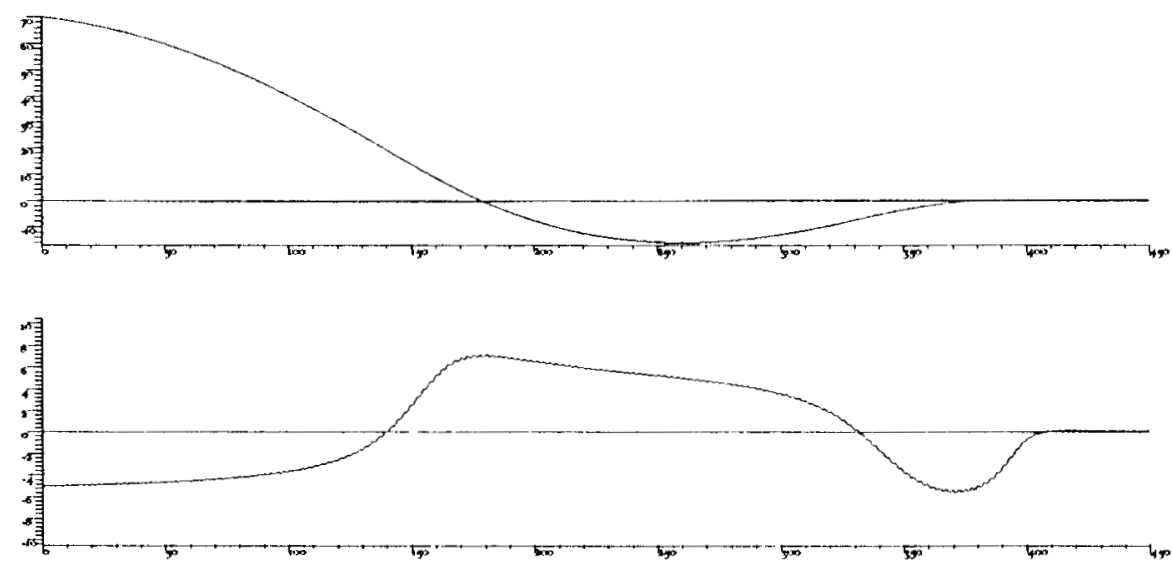

Figure 0.4: Ejemplo 6.2. Evolución de los estados del puente de carga (crane) (4.54) correspondientes a la función RCA rediseñada (4.57) con $\varepsilon=0.1$, arriba; y el desempeño del control, abajo; para la condición inicial $\bar{x}=(70,-0.1,0.003,0)$. 



\section{ESTABILIZACIÓN DE SISTEMAS NO LINEALES MEDIANTE CONTROLES ACO- TADOS: ENFOQUE DE PROGRAMACIÓN PARAMÉTRICA}

\subsection{Introducción}

Consideremos el sistema afín con entradas múltiples

$$
\dot{x}=f(x)+\sum_{j=1}^{m} u_{j} g_{j}(x),
$$

donde $x \in \mathbb{R}^{n}, f, g_{j}: \mathbb{R}^{n} \rightarrow \mathbb{R}^{n}, j=1, \ldots, m$, son funciones suaves y la entrada $u$ toma sus valores en la bola $p$-normada de radio $r>0, u \in \mathcal{B}_{r}^{p}=\left\{u \in \mathbb{R}^{m}:\|u\|_{p} \leq r\right\}$, donde $\|u\|_{p}:=\left(u_{1}^{p}+\ldots+u_{m}^{p}\right)^{1 / p}$ es una $p$-norma, para $p \in \mathbb{N}$ par. Sin pérdida de generalidad. supondremos que el origen es un punto de equilibrio de la dinámica libre asociada de (5.1), i.e.. $f(0)=0$.

En este capítulo, estudiaremos el problema de la estabilización global asintótica (EGA) del sistema (5.1) mediante una función de retroalimentación de control acotacla (RCA) $u=u(x)$. sujeta a la restricción $\|u(x)\|_{p} \leq r$. A lo largo de este capítulo, la estabilización siempre será entendida como estabilización del origen.

En los últimos años, ha habido un creciente interés con respecto al problema de la estabilización global de sistemas lineales mediante funciones RCA; mientras que, para el caso general de sistemas no lineales (5.1) hay relativamente pocos resultados, que están principalmente orientados a los sistemas bilineales.

El problema anterior dio pauta para la caracterización de una clase de sistemas lineales: Sistema lineal asintóticamente controlable en el origen con controles acotados (ACOCA). Como es bien sabido, un sistema lineal es ACOCA ssi es estabilizable por controles arbitrariamente pequeños ( $c f$. Sontag [56] y las referencias ahí contenidas). Este problema de estabilización ha sido abordado mediante dos metodologías principales: (i) Diseñar controles no lineales basados en retroalimentaciones lineales saturadas (RLS) o (ii) diseñar controles no lineales más generales. Para la primera metodología ( $i$ ), la propuesta natural de tomar una RLS simple se probó fallida para obtener la EGA de la clase de sistemas ACOCA (en específico, el $n$-integrator $(n \geq 3)$ Fuller (12|), aunque este resultado si puede obtenerse con la clase general de las funciones RCA (cf. por ejemplo [57]-[60]-[6.1]). En vista de este resultado negrativo, Sussmum-SontagYang [64] generalizaron un método debido a Teel [65] (el enfoque de saturaciones anidadas), 
para obtener la EGA de los sistemas lineales ACOCA mediante combinaciones lineales y composiciones anidadas de RLS. Respecto a la segunda metodología (ii), Komarov [2T] usó una estimación elipsoidal de los conjuntos controlables de sistemas lineales, pues estaba interesado en una aproximación suave del problema de control en tiempo óptimo. Un parámetro de tal aproximación elipsoidal se obtiene resolviendo una ecuación diferencial matricial no lineal. El estabilizador resultante es una función RCA de tiempo finito subóptima, que es suave excepto en el origen. Empero, la región donde el control está definicio no está bien deterninada. Basándonos en el enfoque de Komarov, en el Capítulo 2 (o [62]), establecimos una conexión entre la estabilización asintótica y la de tiempo finito vía un rediseñamiento del control, y además obtuvimos una función RCA. Ese capítulo contiene también una solución al problema de EGA para el $n$-integrator.

En el Capitulo 3 (o [60]) introdujimos una técnica que proporciona un resultado de asignación de eigenvalores para sistemas lineales con controles acotados. Mediante ese método construimos una función RCA que permite: (i) la EGA de los sistemas lineales ACOCA: y (ii) para los sistemas lineales controlables cuyas dinámicas libres son inestables, da lugar a una estimación interna de la región de atracción. En ese capítulo, partimos de la teoría LQ para derivar una retroalimentación estabilizante global, cuyas ganancias dependen del tamaño de los estados (ganancia programada). Para este propósito, se debe resolver una familia $\tau$-parametrizada de ecuaciones matriciales algebraicas (o diferenciales) de Riccati para obtener el control.

Es importante hacer mención que en las dos metodologías ([27]-[62] y [60]), el sistema a lazo cerrado resultante está definido implícitanente, en el sentido de que consiste de un sistema de ecuaciones diferenciales más una ecuación algebraica no lineal. Además, los controles son estabilizadores de tipo lineal de la forma $u(x)=-K(x) x$, cuya ganancia, que depende de los estados $K(x)$, ajusta la amplitud de la entrada de acuerdo al tamaño del estado para satisfacer las cotas sobre la entrada. En este sentido, las dos metodologías pueden concebirse como de ganancias programadas.

Por contraste con el caso de sistemas lineales de control, la caracterización de sistemas no lineales que sean del tipo ACOCA permanece aun como un problema abierto. Además, las estrategias generales que se han propuesto para estabilizar los sistemas no lineales mediante control acotado es una tarea sumamente difícil.

Entre las herramientas que usualmente se emplean para la estabilización de sistemas no lineales se hallan el análisis de Lyapunov, el principio de invariancia de LaSalle, la teoría de la variedad central, y álgebras de Lie [5]-[19]-[23]-[31]-[35]-[33]. A este respecto, Jurdjevic-Quinn (J-Q) [23] desarrollaron un enfoque exitoso, basado en una condición del tipo de rango de controlabilidad definida en términos de álgebras de Lie: la llamada condición ad. En [23], se consideró el caso sistemas afines con entrada escalar cuyas dinámicas libres son lineales y la matriz de transición de estados es unitaria. Para sistemas bilineales con entrada escalar, los siguientes resultados fueron obtenidos bajo la misma línea de la técnica de J-Q: (i) Slemrod [50] propuso una mejora a la condición ad para obtener un control saturado que estabiliza global asintóticamente la clase de sistemas bilineales homogéneos cuya matriz de transición de estados es antisimétrica: y (ii) Gauthier y Kupka [14] investigaron el problema de la EGA de los sistemas bilineales con dinámica libre disipativa, vía leyes de retroalimentación suaves y controles discontinuos. Estos últimos autores también presentaron condiciones para obtener 
la EGA de sistemas no lineales mediante controles retroalimentados suaves arbitrariamente pequeños -una propiedad que caracteriza la clase de los sistemas lineales ACOCA.

También se han propuesto métodos diferentes al enfoque J-Q para la estabilización de sistemas bilineales. Mohler [43] abordó el problema de la estabilización de sistemas bilineales con control escalar usando controles de tiempo óptimo (bang-bang); mientras que Longchamp [39] investigó la estabilización mediante controles bang-bang que determinan una condición de modo deslizante (sliding mode). Finalmente, para los sistemas bilineales con entradas múltiples, Gutman [17] obtuvo estabilizadores incluso cuando los eigenvalores de la dinámica lineal libre tienen parte real positiva, e introdujo un esquema de control acotado para sistemas bilineales diádicos, el control división.

Recientemente, sobre la línea de investigación de [14], Lin [34] obtuvo la EGA de cierta clase de sistemas afines con entradas múltiples, cuya dinámica libre es lineal y Lyapunov estable, bajo la consideración tanto de funciones retroalimentadas suaves (no acotadas) o acotadas ("estabilizadores pequeños" discontinuos). Lin derivó una condición ad, que generaliza los resultados obtenidos por Lee-Arapostathis [31] y Byrnes-Isidori-Willems [5], y que mejora la de J-Q. En Lin [35], el método previo fue extendido para obtener la EGA para la clase general de sistemas no afines pasivos. Dado que los sistemas pasivos tienen la propiedad tipo ACOCA [35] antes mencionada, éstos pueden perisarse como sistemas no lineales "casi lineales". Para más propiedades sobre esta clase importante de sistemas, cf. [5]-[19]-[70].

Es importante notar que en los métodos antes mencionados, los controles son bien sea (i) del tipo de baja ganancia -que implica que la estabilización global asintótica se obtiene a expensas de variaciones lentas de la función de control, y por ende obteniendo (probablemente) un pobre desempeño y largos tiempos para terminar (settling times), o bien (ii) controles bang-bang -que pueden concebirse como controles con ganancias infinitas, lo cual impide sus usos en algunas aplicaciones.

En este capítulo, se presenta un enfoque de diseño que permite vía un control retroalimentado acotado, la estabilización global asintótica de sistemas afines cuyas dinámicas libres son Lyapunov estables. Como mencionamos antes, en el Capítulo 3 (o [60]), diseñamos un control acotado basado en la teoría $L Q$ con ganancias dependientes de los estados para la estabilización global asintótica de los sistemas lineales ACOCA. Con base en las ideas básicas usadas en los Capítulos 3 y 4 , la ley de control (de alta ganancia) que proponemos en este capítulo, incrementa la ganancia a medida que la trayectoria controlada converge hacia el origen, con lo cual se garantiza que las cotas sobre la entrada no sean excedidas. Crosso modo, el procedimiento consiste en definir cierta función $(\tau)$, tal que sea constante a lo largo de las fronteras de los conjuntos de nivel $c$ de la función de Lyapunov asociada con el sistema sin control. Entonces, si tomamos esos conjuntos de nivel $c$ en una sucesión de tarnaño decreciente (que converge al origen), se asigna a cada conjunto tal la mayor ganancia posible correspondiente (vía $\tau$ ), bajo la condición de que el control se mantenga acotado. En general, ese procedimiento implica que las ganancias, como funciones de la posición, se obtienen de la solución de un problema de programación no lineal c-parametrizado.

Con especial interés se estudia una clase importante de sistemas homogéneos y sistemas bilineales. Respecto a la primera clase, la ley de control construida está explícitamente definida. En el caso general no lineal (e.g., los sistemas bilineales no homogéneos), el sistema a lazo 
cerrado resultante está implícitamente definido, en el sentido de que consiste de un sistema de ecuaciones diferenciales más una ecuación algebraica no lineal (requerida para obtener el control). El control diseñado es una función de retroalimentación suave, excepto en el origen (que es una singularidad). En vista de que para un amplio espectro de aplicaciones, ésta es una característica indeseable (e.g., causa del llamado chattering del controi), siempre es posible obtener un estabilizador continuo asintótico mediante un rediseñamiento del control.

Dependiendo de algunas aplicaciones, e.g. robot manipuladores, procesos químicos de control, etcetera, el considerar que la razón de cambio sobre el control es ilimitada puede concebirse como una desventaja de la mayoría de lo métodos existentes para el diseño de estabilizadores acotados: el control por lo regular semeja un control tipo bang-bang, especialmente cuando los estarlos se hallan lejos del origen. Ese comportamiento excluye su uso en las mencionadas aplicaciones. debido a las respuestas naturales (e.g. inercia del actuador) de estos sistemas a estímulos extemos. Comparado con esos métodos, una cualidad remarcable de mestra técnica recae en la "suavidad" de la derivada de los controles acotados. El método se aplica exitosamente a la clase mencionada de sistemas homogéneos (que a su vez incluye a la clase de sistemas que pueden ser estabilizados global y asintóticamente por retroalimentaciones lineales), y además se aborda el problema cuando las entradas están sujetas a cotas globales en sus derivadas.

En muchas aplicaciones, el conjunto de restricciones del control se define como el $r$-hipercubo $m$-dimensional (la bola $\infty$-nomada) $\mathcal{B}_{r}^{\infty}=\left\{u \in \mathbb{R}^{m}:\|u\|_{\infty}:=\max _{i}\left|u u_{i}\right| \leq r\right\}$. Empero, si ésta es considerada, el problema de programación $c$-parametrizado sería no suave, y por ende muy difícil de resolver. Por lo tanto, se introduce aquí una bola de radio $r>0$ vía la norma $p$, $\mathcal{B}_{r}^{p}=\left\{u \in \mathbb{R}^{m}:\|u\|_{p} \leq r\right\}$ de forma tal que, al incrementar $p$, se pueda obtener cualquier grado de aproximación suave al conjunto de restricciones del control $\mathcal{B}_{r}^{\infty}$. Por consiguiente, se obtiene así una utilización bastante buena del recurso (magnitud) disponible de control.

\subsection{Preliminares y planteamiento del problema}

Recordemos que una función $V: \mathbb{R}^{n} \rightarrow \mathbb{R}$ se dice definida positiva ssi $V(0)=0$ y $V(x)>0$, para toda $x \neq 0$; y propia ssi, para cualquier $c \geq 0$, el conjunto $V^{-1}(c)=\left\{x \in \mathbb{R}^{\prime 2}: V(x)=c\right\}$ es compacto. En general, $L_{g_{j}} V(x)$ denota la derivada de Lie de la función $V(x)$ en la dirección de la función $g_{j}(x)$. Si $g$ denota la matriz $g(x)=\left(g_{1}(x), \ldots, g_{m}(x)\right), g_{j}: \mathbb{R}^{n} \rightarrow \mathbb{R}^{n}, j=1, \ldots, m$, entonces definimos $L_{g} V(x):=\left(L_{g_{1}} V(x), \ldots, L_{g_{m}} V(x)\right)^{\top}$.

En este capítulo, supondremos la siguiente.

Hipótesis H1. Supongamos que existe una función $C^{k}(h \geq 2) V: \mathbb{R}^{n} \rightarrow \mathbb{R}$, que es definida positiva y propia en $\mathbb{R}^{n}$, tal que el sistema a lazo abierto $((5.1)$ con $u=0) \dot{x}=f(x)$ es Lyapunor estable, i.e.

$$
L_{f} V(x) \leq 0
$$


Elijamos una salida artificial $y:=\left(L_{g} V(x)\right)^{\top}$ para el sistema (5.1). Entonces, el sistema de entrada-salida

$$
\begin{aligned}
& \dot{x}=f(x)+g(x) u \\
& y=h(x)=\left(L_{g} V(x)\right)^{\top},
\end{aligned}
$$

es pasiro [5]-[35] con función de almacenamiento $V(x)$. En efecto, como $L_{f} V(x) \leq 0$ (de la hipótesis $\mathrm{H} 1$ ), se sigue que,

$$
\dot{V}=L_{f} V(x)+\left(L_{g} V(x)\right) u \leq y^{\top} u,
$$

lo cual implica la pasividad del sistema de entrada-salida (5.3) [5]-[35].

Adenás de la hipótesis H1, supondremos que el sistema (5.1) satisface la siguiente.

Hipótesis H2. El sistema de entrada-salida (5.3) es detectable en cero (DEC) [35]. Es decir, para toda $x \in \mathbb{R}^{n}$,

$$
\text { si } \forall t \geq 0,\left.h\left(x\left(t, x_{0} ; 0\right)\right)\right|_{u=0}=0 \text {, entonces } \lim _{t \rightarrow \infty} x\left(t, x_{0} ; 0\right)=0,
$$

donde $x\left(t, x_{0} ; u\right)$ es una trayectoria de $(5.3 \mathrm{a})$ tal que $x(0)=x_{0}$.

Podemos establecer el siguiente resultado.

Proposición 2.1. [5] Supongamos que el sistema (5.1) satisface las hipótesis H1 y H2. Entonces, para cualquier $\rho>0$, la función de control

$$
u(x)=-\rho y=-\rho\left(L_{g} V(x)\right)^{\top},
$$

estabiliza global asintóticamente al sistema (5.1).

A lo largo de este capítulo, supondremos válidas las hipótesis $\mathrm{H} 1$ y $\mathrm{H} 2$, y $y=h(x):=$ $\left(L_{g} V(x)\right)^{\top}$ denotará la salida artificial (5.3b), a menos que se especifique lo contrario.

Observación 1. La prueba de la proposición anterior se basa en el Principio de Invariancia de LaSalle [j]; en virtud de lo cual, la DEC se convierte en una condición suficiente para que el origen sea el máximo conjunto invariante contenido en $\left\{x \in \mathbb{R}^{n}: d V(x) / d t=0\right\}$. Además, en [5], se presenta un criterio (en términos de corchetes de Lie) para la DEC que establece una relación entre detectabilidad y algunas versiones no lineales de criterios de accesibilidad (e.g., $[23]-[24]-[31])$.

En [34]-[35], Lin proveyó un criterio computable para verificar la DEC, tanto para los sistemas afines como los no afines. Ese criterio es una mejora sobre los obtenidos previamente en [5] y [31]. Introduzcamos la distribución

$$
D=\operatorname{gen}\left\{\operatorname{ad}_{f}^{i} g(x): 0 \leq i \leq n-1\right\} .
$$

Sean $\Omega$ y $S$ los conjuntos asociados con $V(x)$,

$$
\begin{aligned}
& \Omega:=\left\{x \in \mathbb{R}^{n}: L_{f}^{i} V(x)=0,1 \leq i \leq h\right\} \quad y \\
& S:=\left\{x \in \mathbb{R}^{n}: L_{f}^{i} L_{d} V(x)=0, \forall d \in D, 0 \leq i \leq k-1\right\} .
\end{aligned}
$$


Entonces, una condición suficiente (test) para que la hipótesis H2 sea válida es la siguiente.

Hipótesis H2'. La intersección de $\Omega$ y $S$ es el conjunto unitario que contiene al origen, i.e.

$$
\Omega \cap S=\{0\}
$$

Hasta ahora, el control dado en (5.6) no es (necesariamente) acotado. Empero, en el caso de los sistemas pasivos, Lin dio el siguiente resultado para estabilización (del tipo ACOCA).

Teorema 2.2. [35] Supongamos válidas las hipótesis H1 y H2. El sistema (5.1) es global asintóticamente estabilizable mediante controles retroalimentados suares arbitrariamente pequeños.

Así. uno puede usar la técnica de saturación suave para obtener una función RCA globalmente definida que estabiliza global asintóticamente estos sistemas. En particular, Lin [35] propuso la siguiente modificación del control (5.6)

$$
\begin{aligned}
& u(x)=-\rho(x) h(x), \\
& \rho(x)=\frac{2 r}{1+\|h(x)\|_{2}^{2}}
\end{aligned}
$$

que da lugar a una función RCA (5.10), tal que $\|u(x)\|_{2}<r$, para cualquier $r>0$ (aquí, $\|\cdot\|_{2}$ es la norma euclideana usual en $\mathbb{R}^{m}$ ). La función de control (5.10) estabiliza global asintóticamente los sistemas afines no lineales (5.1) [35]. Resulta importante observar que este control es del tipo de baja ganancia, i.e., obtenemos una estabilización global asintótica a expensas de variaciones lentas de la función de control. Los controles de baja ganancia pueden dar como resultado respuestas "perezosas" y un bajo desempeño para todas las condiciones iniciales, incluso aquellas cercanas al origen.

Una forma de obtener un control de alta ganancia consiste en incrementar la función $\rho(x)$ a medida que se reduzca la distancia al origen, de manera tal que el control use la máxima amplitud de la entrada sin exceder la restricción de la misma. El objetivo de este capítulo es dar una metodología para diseñar la ganancia del control como una funcion de la posición, de forma que se emplee todo el recurso (magnitud) disponible de control. La idea central es anmentar $p(x)$ cuando la trayectoria se aproxima al origen. En el diseño propuesto, $p(x)$ es constante a lo largo de las fronteras de los conjuntos de nivel de la función de Lyapunov asociada al sistema a lazo abierto.

\subsubsection{Ideas geométricas preliminares}

Con el objeto de aclarar las ideas geométricas sobre las que está basado muestro enfoque de diseño, presentamos la estabilizacion de (5.1) mediante una retroalimentación escalir (5.6) con un parámetro constante $\rho>0$. Así, en la siguiente sección mostraremos cómo construir una función de los estados $p(x)$, que proporciona una función RCA y buenas razones de convergencia para el sistema a lazo cerrado. 
Denotemos por $U(\rho)$ la componente (subconjunto conexo) del conjunto definido por

$$
\left\{x \in \mathbb{R}^{n}:-r \leq-\rho h(x) \leq r\right\}
$$

tal que $0 \in \operatorname{int} U(\rho)$. El conjunto $U(\rho)$ es no vacío. Las fronteras de $U(\rho)$ están dadas por los siguientes conjuntos

$$
\begin{aligned}
& H_{ \pm}=\left\{x \in \mathbb{R}^{n}: \gamma^{ \pm}(x)=0\right\} \\
& \gamma^{ \pm}(x):=-h(x) \pm \frac{r}{\rho} .
\end{aligned}
$$

Como $\partial \gamma^{+} / \partial \rho>0$ y $\partial \gamma^{-} / \partial \rho<0$, entonces es evidente que

$$
U\left(\rho_{2}\right) \subset U\left(\rho_{1}\right), \text { para } \rho_{1}<\rho_{2} \text {. }
$$

Si consideramos la saturación del control (5.6) para $\rho>0, U(\rho) \subset \mathbb{R}^{n}$ es la región donde el contiol no se satura. Si $\rho$ crece, entonces la región de no saturación del control $U(\rho)$ decrece, mientras que si $\rho$ tiende a cero, tenemos

$$
\lim _{\rho \rightarrow 0} U(\rho)=\mathbb{R}^{n}
$$

y se recupera la dinámica del sistema a lazo abierto.

Denotemos por $\mathcal{E}(c)$ la componente del conjunto nivel $c(c>0)$ tal que $0 \in \operatorname{int} \mathcal{E}(c)$, correspondiente a la función de Lyapunov $V(x)$ considerada en la hipótesis $\mathrm{H} 1$, del conjunto

$$
\left\{x \in \mathbb{R}^{n}: V(x) \leq c\right\} \text {. }
$$

Como $U(\rho)$ es no vacío, existe un conjunto máximo $\mathcal{E}\left(c^{*}\right)$, donde $c^{*}$ depende de $p$. contenido en $U(\rho)$. El conjunto $\mathcal{E}\left(c^{*}\right)$ es invariante bajo las trayectorias del sistema a lazo cerrado (5.1)(5.6), luego todas las trayectorias que empiezan en $\mathcal{E}\left(c^{*}\right)$ convergen asintóticamente a 0, mientras que el control queda acotado por $r$. De esta manera, $\mathcal{E}\left(c^{*}\right)$ es un estimado de la región de atracción del origen, $\Omega(0)$, basado en la función de Lyapunov $V(x)$. Además, a partir de (5.4) y $(5.6)$, tenemos

$$
\dot{V}=L_{f} V(x)-\rho h^{2}(x) \leq-\alpha \rho,
$$

donde $\alpha=\max _{x} h^{2}(x)$, para $x \in \mathcal{E}\left(c^{*}\right)$ (recordemos que $V(x)$ es propia). Para mejorar la convergencia, debemos incrementar el valor de $\rho$. Sin embargo, debido a (5.13) el valor de $c^{*}(\rho)$ se reduce (y por ende, el tamaño de $\mathcal{E}\left(c^{*}\right)$ ). Por el contrario, si $\mathcal{E}\left(c^{*}(\rho)\right.$ ) es mayor, las trayectorias convergerán más lento. Aquí surge el dilema: ¿Cómo diseñar el parámetro r como función de la posición a fin de obtener una buena razón de convergencia y estabilidad global con un control acotado? Este problema será estudiado en la siguiente sección.

\subsection{Resultados generales}

Cualquier $x_{0} \in \mathbb{R}^{n}$ puede llevarse asintóticamente a 0 mediante una elección adecuada del parámetro $\rho$ en el control (5.6). En efecto, sólo se requiere buscar un valor de $c=c(\rho)$ tal que

$$
x_{0} \in \mathcal{E}(c) \subset U(\rho) .
$$


En virtud de que $\mathcal{E}(c)$ es un conjunto de nivel (invariante) de $V(x)$. la trayectoria $x_{t}\left(x_{0}\right)$ del sistema (5.1)-(5.6) convergerá asintóticamente a 0. Por otra parte, la inclusión $\mathcal{E}(c) \subset U(\rho)$ asegura que el control $u(x)=-\rho h(x)$ no se saturará para toda $t \geq 0$. Además, toda condición inicial puede llevarse a 0 mediante (5.6) debido a (5.14). En este sentido, podemos decir que el sistema (5.1) es semiglobamente estabilizable por (5.6) con $\rho$ constante. Como es bien sabido, esto significa que para cualquier $x_{0} \in \mathbb{R}^{n}$, podemos elegimos $\rho>0$ tal que $x_{0} \in \Omega(0)$-la región de atracción del origen. No obstante, mediante este enfoque, el sistema es semiglobalmente estabilizado a expensas de un control de baja ganancia.

Resminiendo la discusión en curso, y retonando el caso de múltiples entradas, el mejor diseño semiglobal consiste en elegir el mayor valor de $\rho$ de forma tal que el control $u(x)=-\rho h(x)$ nunca se sature a lo largo de la trayectoria $x_{t}\left(x_{0}\right)$, i.e.. ctado $x_{0}$ se elige $\rho$ de manera que

$$
c^{*}=V\left(x_{0}\right)
$$

y $\mathcal{E}\left(c^{*}\right)$ es el mayor conjunto de nivel contenido en $U(\rho)$. Este problema puede plantearse como el problema de programación

$$
\begin{gathered}
\max \rho \geq 0 \\
\text { t.q. } \quad \mathcal{E}(c) \subset U(\rho) .
\end{gathered}
$$

Con el objeto de simplificar la presentación, definamos $\tau=1 / \rho$. Entonces, el problema anterior puede parafrasearse como

$$
\begin{gathered}
\min \tau \geq 0 \\
\text { t.q. } \frac{1}{r}\|h(x)\|_{p} \leq \tau \quad \text { y } \quad V(x) \leq V\left(x_{0}\right),
\end{gathered}
$$

Dado que $V(0)=0<V\left(x_{0}\right)$, para $x_{0} \neq 0$, es fácil ver que si existe una solución $\rho^{*}=1 / \tau^{*}$ al problema (5.20), entonces $\mathcal{E}\left(c^{*}\right)=\mathcal{E}\left(V\left(x_{0}\right)\right) \subset U\left(p^{*}\right)$. Así, $\rho^{*}$ será el mayor valor de $\rho$ que permite la convergencia asintótica al 0 de la trayectoria $x_{t}\left(x_{0}\right)$ con la máxima razón de convergencia respecto a la función de Lyapunov $V(x)$, y al mismo que el control $u(x)=-p^{*} h(x)$ permanece acotado, para toda $t \geq 0$.

Podemos obtener una formulación equivalente más simple que (5.20) como sigue. Elijamos $x_{0} \neq 0$ arbitraria y hagamos $c=V\left(x_{0}\right)(>0)$. Si tomamos en consideración la condición (de frontera) $u \in \partial U$, i.e. $\tau=\frac{1}{r}\|h(x)\|_{p}$, obtenemos

$$
\begin{gathered}
\tau\left(x_{0}\right)=\frac{1}{r} \max _{x}\|h(x)\|_{p} \\
\text { t.q. } x \in \partial \mathcal{E}(c), \quad \text { donde } c=V\left(x_{0}\right) .
\end{gathered}
$$

El problema de optimización (5.21) es una familia de programas uno-paramétrica $(c \geq 0)$ con una restricción de igualdad variable [10]-[22], cuya función objetivo $\|h(x)\|_{\text {, }}$ no depende del parámetro c. En particular, como $V$ es propia (por la hipótesis $\mathrm{H1}$ ), la familia de programas dada arriba es propia [10]. Un programa propio (como (5.21)) tiene al menos una solución global para cada $c \in \mathbb{R}$, pues el conjunto $V^{-1}(c)(=\partial \mathcal{E}(c))$ es compacto. Con el objeto de exchuir el caso en que $\partial \mathcal{E}(c)$ sea un conjunto discreto para toda $c>0$, restringiremos el problema suponiendo que $n \geq 2$. Es decir, no consideramos a los sistemas uno-dimensionales. 
Definamos la función de control $\tau$-dependiente

$$
u_{\tau}(x):=-\frac{1}{\tau(x)} h(x)
$$

Definición. Una solución $\tau=\tau(x)$ del problema de optimización (5.21) se denominará admisible ssi satisface las siguientes propiedades:

(a) $\tau(x)$ es definida positiva; $y$

(b) la igualdad $S_{\tau^{*}}=\left\{x \in \mathbb{R}^{n}: \tau(x) \leq \tau^{*}\right\}=\mathcal{E}\left(c\left(\tau^{*}\right)\right)$ se satisface, para cada $\tau^{*} \geq 0$. Así, $S_{\tau^{*}}$ es in conjunto invariante bajo las trayectorias del sistema a lazo cerrado (5.1)-(5.22).

Si $\tau(x)$ es una solución admisible, el control $u_{\tau}(x)(5.22)$ es suave excepto en el origen, donde es singular (i.e., no está definido en $x=0$ ). Luego, el origen es más bien una singularidad en lugar de un punto de equilibrio del sistema a lazo cerrado (5.1)-(5.22). Esto significa que este sistema no es Lipschitz en el origen (no unicidad de las solutions respecto a condiciones iniciales), así todas las trayectorias podrían converger a $x=0$ en un tiempo finito. Para un gran número de aplicaciones, ésta es una característica que puede llevar a un comportamiento indeseable, e.g. causar el llamado "chicoteo" (chattering) del control. Por ello, esta ley de control debe redefinirse para obtener la siguiente función $\mathrm{RCA}$ asintótica (diferenciable excepto en $\partial \mathcal{E}\left(\tau^{\times}\right)$)

$$
u^{\times}(x)=\left\{\begin{array}{ll}
u_{\tau}(x), & \text { si } x \in \mathbb{R}^{n} \backslash \mathcal{E}\left(\tau^{\times}\right) \\
-\frac{1}{\tau^{\times}} h(x), & \text { si } x \in \mathcal{E}\left(\tau^{\times}\right)
\end{array},\right.
$$

conde $\tau^{\times}>0$ es arbitrario, que estabiliza asintóticamente al sistema afín (5.1).

Proposición 3.1. Sobre la base de las hipótesis $\mathrm{H} 1$ y H2, si $\tau(x)$ es una solución admisible para el problema de optimización (5.21), entonces para cualquier $\tau^{\times}>0$, el control (5.23) satisface $\left\|u^{\times}(x)\right\|_{p} \leq r$ y el sistema a lazo cerrado (5.1)-(5.23) es EGA.

Prueba. Como $\tau(x)$ es una solución del problema de optimización, entonces $\left\|u^{\times}(x)\right\|_{p} \leq r$. Por otra parte, tomando en cuenta a la hipótesis $H 1$, que $\tau(x)$ es admisible, y si consideramos el sistema a lazo cerrado (5.1)-(5.23), entonces para toda $x \in \mathbb{R}^{n}$, obtenemos

$$
\dot{V}(x)=\left\{\begin{array}{ll}
L_{f} V(x)+h^{\top}(x) u_{\tau}(x), & \text { si } x \in \mathbb{R}^{n} \backslash \mathcal{E}\left(\tau^{\times}\right) \\
L_{f} V(x)-\frac{1}{\tau^{\times}} h^{\top}(x) h(x), & \text { si } x \in \mathcal{E}\left(\tau^{\times}\right)
\end{array},\right.
$$

es no positiva en ambos casos. Luego, el punto de equilibrio $x=0$ del sistema a lazo cerrado es estable. A fin de probar que éste es EGA, denotemos con $I=\left\{x \in \mathbb{R}^{n}: d V / d t=0\right\}$. Entonces, para toda $x \in I$, de (5.24), obtenemos

$$
L_{f} V(x)=0 \quad \text { y } \quad h_{j}(x)=L_{g_{j}} V(x)=0, j=1, \ldots m .
$$

La afirmación se sigue en términos de la hipótesis $\mathrm{H} 2$, adaptando la prueba del Principio de Invariancia de LaSalle para mostrar que $\{0\}$ es el mayor conjunto invariante contenido en $I$. Cf. la prueba del Teorema 3.2 en [5], para mayores detalles. 
Observación 2. Debemos remarcar que, tal como está planteaco, el problema de optimización (5.21) no es suave sobre el conjunto $\left\{x \in \mathrm{R}^{n}: h(x)=L_{g} V(x)=0\right\}$. Esta dificultad se puede evitar si la función $\|h(x)\|_{p}^{p}$ se usa como función objectivo.

Propiedades de la función $\tau$ :

(i) $\tau(x)$ está bien definida en $\mathrm{R}^{n}$, pues (5.21) es propio; $\mathrm{y}$

(ii) $\tau(x) \geq 0$.

Debiera resultar claro que el control (5.22) es suave, siempre que la función $\tau(x)$ lo sea. De la observación 2, tenemos que esta propiedad importante (la diferenciabiliclad) de $\tau(x)$ está garantizada si se satisfacen los dos puntos siguientes:

- la suavidad de $\tau^{p}(x)$, definida como la solución de $(5.21)$ con $\|h(x)\|_{p}^{p}$ usada como función objectivo; y

- que $\tau(x)$ sea definida positiva.

Para el problema de optimización general, los resultados de suavidad para la función $\tau^{p}(x)$ son de carácter genérico, cf. el Apéndice. Aparte de su obvia relevancia en evitar singularidades (además de $x=0$ ) en el control (5.22) y el resultado de estabilidad de la Proposición 3.1, el segundo punto resalta la importancia de requerir que $\tau$ sea definida positiva. Por lo tanto, en lo que resta de esta sección, atenderemos exclusivamente este áltimo problema.

Observación 3. La propiedad que $\tau(x)$ sea definida positiva puede reducirse a las siguientes equivalencias: $\tau(x)=0 \Longleftrightarrow\left\|h\left(x^{*}\right)\right\|_{p}=0$, donde $x^{*}$ es una solución óptima de $(5.21) \Longleftrightarrow$ para toda $x \in \partial \mathcal{E}(c)$, donde $c=V\left(x^{*}\right) \geq 0$, tenemos $\|h(x)\|_{p}=0$ (pues $\left.\|h(x)\|_{p} \leq\left\|h\left(x^{*}\right)\right\|_{p}=0\right) \Longleftrightarrow$ para toda $x \in \partial \mathcal{E}(c), L_{g} V(x)=0 \Longleftrightarrow$ el conjunto de campos vectoriales $\left\{g_{j}(x), j=1, \ldots, m\right\}$ es tangencial al conjunto compacto $\partial \mathcal{E}(c)$.

De aquí en adelante, en virtud de la observación anterior, supondremos la signiente.

Hipótesis H3. Supongamos que $n>1$ y además $L_{g} V(x)$ no se anula en toda la frontera de un conjunto de nivel $\partial \mathcal{E}(c)$ (excepto en $c=0$ ), i.e.,

$$
\nexists c>0, \forall x \in \partial \mathcal{E}(c), \quad L_{g} V(x)=0
$$

La hipótesis anterior no es demasiado restrictiva, dado que el conjunto de campos vectoriales que son transversales a una función propia fija ( $V(x)$ en este caso) es denso y abierto, con respecto a la topología de Whitney, dentro del conjunto de todos los campos vectoriales definidos en $R^{n}$. Esta afirmación se sigue de un problema difícil (aunque cierto) propuesto en el libro de Hirsch [20] y, hasta donde sabemos, su prueba fue finalmente presentada en [74] para el caso generalizado de $k$-jets. Consecuentemente, $\tau(x)$ es definida positiva, y por encle una función admisible, en un sentido genérico.

La función $\tau(x)$ puede probarse admisible en ciertos casos particulares, aunque importantes. Proposición 3.2. Considereremos el sistema (5.1) y $n>1$. Entonces. $\tau(x)$ es una función admisible en los siguientes casos especiales: 
(a) si $g(x)=B=\left(b_{j}\right)$-una matriz constante $n \times m$ :

(b) si $g_{j}(x), j=1, \ldots, m$, y la función de Lyapunov asociada $V(x)$ son homogéneas;

(c) si $g(x)=d(x)+b$, clonde $0 \neq b \in \mathbb{R}^{n}$-constante $y d_{j}(x)$ son funciones impares para $j=1, \ldots, m, y$ el sistema a lazo abierto tiene una función de Lyapunov par $V(x)$.

Prueba. Los tres puntos pueden probarse por reductio ad absurdum: Supongamos que existe $c>0$ tal que $\left.\tau(x)\right|_{\partial \varepsilon(c)} \equiv 0$.

Caso (a): En este caso, el conjunto de sistemas $\dot{x}=g_{j}(x)=b_{j}, j=1, \ldots, m$, tiene soluciones paralelas, y así $\Delta=\bigcap_{j=1}^{m} \operatorname{ker} b_{j}$ es un subespacio lineal con $\operatorname{dim} \Delta<n$. Por otra parte, para cada $c>0$, el conjunto de nivel $\mathcal{E}(c)$ satisface: int $\mathcal{E}(c) \neq \emptyset$ (debido a la continuidad de $V(x)$ ) $y$ el conjunto (frontera) de nivel $\partial \mathcal{E}(c)$ es compacto (debido a que $V(x)$ es propia). Por lo tanto, el conjunto de campos vectoriales $\left\{b_{j}\right\}, j=1, \ldots m$, no puede ser tangencial a todo el conjunto compacto $\partial \mathcal{E}(c)$, a menos que $\partial \mathcal{E}(c) \subset \Delta$, pero esto es una contradicción.

Caso (b): En vista de que $V(x)$ es definida positiva y $c>0$, entonces $0 \in \operatorname{int} \mathcal{E}(c) \neq 0$. Debido a la compacidad $\mathcal{E}(c)$, un argumento de continuidad muestra que dado cualquier $y \in \mathbb{R}^{\prime 2}$, existen $x \in \partial \mathcal{E}(c)$ y $\eta>0$ tales que $y=\eta x$. De donde, como $g_{j}(x), j=1, \ldots, m$, y $V(x)$ son funciones homogéneas de grados $d_{j}$ y $s$, respectivamente, debemos obtener que para toda $y \in \mathbb{R}^{n}$, $-h_{j}(y)=-L_{g_{j}} V(y)=-\eta^{d_{j}+s-1} L_{g_{j}} V(x)=0, j=1, \ldots, m$. Consecuentemente, si suponemos que $\tau(x)$ se anula en toda la frontera de un conjunto de nivel $\partial \mathcal{E}(c)$, entonces o bien $g(x) \equiv 0$ o bien $V(x) \equiv 0$ en todo el espacio $\mathbb{R}^{n}$.

Caso (c): En este caso, el conjunto de campos vectoriales $g_{j}(x)=d_{j}(x)+b_{j}, j=1, \ldots, m$, debiera ser tangencial a todo el conjunto simétrico $\partial \mathcal{E}(c)=\left\{x \in \mathbb{R}^{n}: V(x)=c\right\}$. En efecto, supongamos que para toda $x \in \partial \mathcal{E}(c),-h_{j}(x)=-L_{g_{j}} V(x)=-\nabla V(x)\left(d_{j}(x)+b_{j}\right)=0$, $j=1, \ldots, m$. Sin embargo, por simetría, $-x \in \partial \mathcal{E}(c), y$ así, $-h_{j}(-x)=-L_{q_{j}} V(-x)=$ $-2 \nabla V(x) b_{j}=0, j=1, \ldots, m$, pero esto contradice el Caso (a).

Observación 4. Otra reformulación del control (5.22), se obtiene mediante la siguiente función RCA globalmente asintótica. Definamos la función de control $(\tau, \varepsilon)$-dependiente

$$
u_{\bar{\varepsilon}}(x):=-\frac{1}{\varepsilon+\tau(x)} h(x)
$$

donde $\varepsilon>0$ es un parámetro de sintonía suficientemente pequeño. Es fácil probar que $u_{\Sigma}(x)$ estabiliza global asintóticamente el sistema (5.1). Observemos que el control (5.27) nunca se satura, i.e. $\left\|u_{\xi}(x)\right\|_{p}<r$, lo cual significa que este control (5.27) no usa todo el recurso disponible. Por otra parte, para (la clase "magra" de) los sistemas restantes parra los que $\tau(x)$ no es definida positiva, (5.27) puede ser usado en lugar de (5.22).

Observación 5. Aunque el control redefinido (5.27) es no singular, vale la pena mencionar que éste pierde su diferenciabilidad siempre que la función $\tau(x)$ se anula. Esto se torna en contra de la suavidad deseada, y por ende el buen desempeño, de ese control.

Observación 6. Un enfoque ingen1o, sugerido por (5.27), radica en considerar la siguiente modificación de la retroalimentación (5.6)

$$
u_{\delta}(x):=-\frac{r}{\delta+\|h(x)\|_{p}} h(x)
$$

donde $\delta>0$, es un parámetro de sintonía suficientemente pequeño. Empero, este control suele dar un desempeño pobre, debido principalmente a la no diferenciabilidad de (5.28) en todos los puntos donde la función $h(x)$ se hace cero. 


\subsection{Método de multiplicadores de Lagrange}

Como planteamos en la sección anterior, el problema de diseño de control se reduce al de hallar una función de ganancias programadas $\tau(x)$. En virtud de $(5.18)-(5.22)$, este último problema es uno de optimización con restricciones inducidas por las cotas de las entradas.

Denotemos con $D g$, la matriz jacobiana de una función $g$, y con $\nabla_{x} f$ y $\nabla_{x x} f$, el gradiente y la matriz hessiana de una función de valores reales $f$ con respecto a $x$, respectivamente. Ln punto $x \in \mathbb{R}^{2}$ es un punto crítico de una función $f$ ssi $\nabla_{x} f(x)=0$, en otro caso se dice cuue $x$ es un punto regular de $f$. Además, si det $\left(\nabla_{x x} f(x)\right) \neq 0$, entonces $x$ se dice que es un punto crítico no degonerado de la función $f$. Un punto $c \in \mathbb{R}$ es un valor regular de $f$. denotado $f \pitchfork c$ ssi todo $x \in f^{-1}(c)$ es un punto regular de $f$.

Una condición necesaria de primer orden para que el problema de optimización 1-paramétrico (5.21) tenga una solución puede darse en términos del método de multiplicadores de Lagrange.

Proposición 4.1. Consideremos la familia de problemas de optimización 1-paramétricos (5.21). Supongamos que para cada $c>0$, existen $\lambda^{*} \in \mathbb{R}$ y una solución óptima $r^{*} \in \mathbb{R}^{n}$ tales que $r^{*}$ es un printo regular de la restricción, $g\left(x^{*}\right)$ tiene rango máximo y $\tau\left(x^{*}\right)$ es definida positiva. Entonces, para cualquier $x \in \partial \mathcal{E}(c)$, la función $\tau(x)$ satisface la siguiente fórmula

$$
\tau^{p-1}\left(\tau-\lambda^{*} y^{* \top} G^{-1} y^{*}\right)=-\frac{1}{p r^{p}} \nabla_{y}\left\|y^{*}\right\|_{p}^{p} D g^{\top}\left(\nabla_{x} V\right)^{\top} g G^{-1} y^{*}
$$

donde $G=g^{\top} \nabla_{x x} V g$ y $y^{*}=h\left(x^{*}\right)$.

Prueba. Elijamos $0 \neq x_{0} \in \mathbb{R}^{n}$, fijemos $c=V\left(x_{0}\right)(>0)$ y sea $x^{*} \in \mathbb{R}^{n}$ una solución óptima al programa propio (5.21). Entonces, por hipótesis y basados en el método de multiplicadores de Lagrange, tenemos que $x^{*}$ es un punto regular de la restricción y existe $\lambda^{*} \in \mathbb{R}$ tal que $x^{*}$ es un punto crítico del programa; es decir, el Jagrangiano asociado

$$
L_{\lambda *}\left(x^{*}\right)=\frac{1}{r}\left\|h\left(x^{*}\right)\right\|_{p}-\lambda^{*}\left(V\left(x^{*}\right)-c\right),
$$

para $c>0$ fija, satisface

$$
\nabla_{x} L_{\lambda} \cdot\left(x^{*}\right)=\frac{\tau^{1-p}}{p r^{p}} \nabla_{y}\left\|y^{*}\right\|_{p}^{p} D h\left(x^{*}\right)-\lambda^{*} \nabla_{x} V\left(x^{*}\right)=0
$$

donde $y^{*}=h\left(x^{*}\right)$. Obviamente, $\|y\|_{p}^{p}$ es una función definida positiva, convexa y homogénea de grado $p$.

Por otra parte,

$$
D h\left(x^{*}\right)=g^{\top}\left(x^{*}\right) \nabla_{x x} V\left(x^{*}\right)+D g^{\top}\left(x^{*}\right)\left(\nabla_{x} V\left(x^{*}\right)\right)^{\top},
$$

donde $g(x)=\left(g_{1}(x), \ldots, g_{m}(x)\right)^{\top}, g_{j}: \mathbb{R}^{n} \rightarrow \mathbb{R}^{n}, j=1, \ldots, m$. Por hipótesis $g\left(x^{*}\right)$ tiene rango máximo y $\tau\left(x^{*}\right) \neq 0$. Entonces, multipliquemos por la derecha ambos lados de (5.31) por $g\left(x^{*}\right)$ y reemplacemos $D h\left(x^{*}\right)$ con (5.32), para obtener

$$
\nabla_{y}\left\|y^{*}\right\|_{p}^{p}\left(G+D g^{\top}\left(\nabla_{x} V\right)^{\top} g\right)-p r^{p} \tau^{p-1} \lambda^{*} y^{* \top}=0
$$


donde $G=g^{\top} \nabla_{x x} V g$. Si la solución óptima $x^{*}$ satisface $\operatorname{det}\left(\nabla_{x x} V\left(x^{*}\right)\right) \neq 0$ y $g\left(x^{*}\right)$ tiene rango máximo. entonces existe $G^{-1}\left(x^{*}\right)$. Además, por (la fórmula de Euler) homogeneidad de $\|y\|_{p}^{p}$. tenemos $\nabla_{y}\|y\|_{p}^{p} y=p\|y\|_{p}^{p}$. Así, si rearreglamos (5.33) y multiplicamos por la derecha ambos lados por $y^{*}$. obtenemos

$$
p\left\|y^{*}\right\|_{p}^{p}=p r^{p} \tau^{p}=\left(p r^{p} \tau^{p-1} \lambda^{*} y^{* \top}-\nabla_{y}\left\|y^{*}\right\|_{p}^{p} D g^{\top}\left(\nabla_{x} V\right)^{\top} g\right) G^{-1} y^{*}
$$

O de manera equivalente (5.29), en donde hemos omitido en (5.33) y (5.34) la dependencia en $x^{*}$, y reemplazado $h\left(x^{*}\right)$ con $y^{*}$, por claridad.

Antes probamos, en la Proposición $3.2($ a), que $\tau(x)$ es una solución admisible en el caso de lus sistomas (5.1) para los que $g(x)=B=\left(b_{j}\right), j=1, \ldots m$. A continuación podemos proporcionar una fórmula explícita para $\tau(x)$ en $(5.36)$.

Corolario 4.2. Consideremos el sistema afín (5.1)

$$
\dot{x}=f(x)+B u,
$$

donde $B$ es una matriz constante $n \times m$ de rango $m$. Entonces, $\tau(x)$ está dada por (5.36). Prueba. De (5.33) y (5.29), obtenemos

$$
\tau(x)=\left(\frac{h^{\top}\left(x^{*}\right) G^{-1}\left(x^{*}\right) h\left(x^{*}\right) \nabla_{y}\left\|h\left(x^{*}\right)\right\|_{p}^{p} G\left(x^{*}\right) h\left(x^{*}\right)}{p h^{\top}\left(x^{*}\right) h\left(x^{*}\right)}\right)^{1 / p},
$$

donde, $x^{*}$ es la solución óptima de (5.21) sujeta a $V(x)=V\left(x^{*}\right)=c$.

Respecto al problema de optimización general, es pertinente introducir algunas restricciones a fin de obtener programas "bien comportados" y resultados de tipo genérico. Con este objeto. se han propuesto los programas de Morse [10] y los programas regulares [22]. Cf. el Apéndice, para mayores detalles sobre este particular.

\subsubsection{El caso de entrada escalar}

En el caso particular de entrada escalar, el problema de optimización (5.21) es equivalente a resolver los siguientes dos programas

$$
\tau\left(x_{0}\right)=\frac{1}{r} \max _{x}|h(x)|=\frac{1}{r} \max \left\{\max _{x} h(x),-\min _{x} h(x)\right\}
$$

sujetos a $x \in \partial \mathcal{E}(c)$, donde $c=V\left(x_{0}\right)$. Este problema puede reducirse a sólo un problema de optimización, si el máximo se alcanza siempre en sólo uno de los programas. Una condición suficiente para esto, procede como sigue: Supongamos que existe $x_{0} \in \partial \mathcal{E}(c)$ tal que $\tau\left(x_{0}\right)=$ $\frac{1}{r} \max _{x} h(x)=-\frac{1}{r} \min _{x} h(x)$, para $x \in \partial \mathcal{E}(c)$. Entonces existen $x_{1}^{*}, x_{2}^{*} \in \partial \mathcal{E}(c)$, tales que $h\left(x_{1}^{*}\right)+h\left(x_{2}^{*}\right)=0$, donde $x_{1}^{*}$ y $x_{2}^{*}$ son soluciones de los programas $x_{1}^{*}=\arg \max _{x} h(x)$ y $x_{2}^{*}=-\arg \min _{x} h(x)$, respectivamente. Con base en la hipótesis H3, tenemos que $\tau(x)$ es definida positiva, luego $x_{1}^{*} \neq x_{2}^{*}$. Entonces, pudiera existir un intervalo abierto $I=(-\varepsilon+c, c+\varepsilon)$, 
$\Xi>0$, tal que si $\Sigma_{I}$ denota el conjunto de soluciones óptimas asociado a (5.37), entonces $\Sigma_{I}$ debiera estar contenido en el correspondiente conjunto de puntos críticos de los programas anteriores. Si este es el caso, $\Sigma_{I}$ bien podría ser una unión ajena de curvas seccionalmente suaves [22], cf. el Apéndice. Por lo tanto, desde la perspectiva de la implementación de un programa, a fin de evitar "brincos" indeseables entre esas curvas, podemos suponer que la función $\psi: \mathbb{R} \rightarrow \mathbb{R}$, definida por

$$
\begin{gathered}
\psi(c):=\max _{x} h(x)+\min _{x} h(x) \\
\text { t.c. } \quad x \in \partial \mathcal{E}(c) .
\end{gathered}
$$

es bien sea no negativa o bien no positiva, para toda $c>0$.

Sin pérdida de generalidad, supongamos que la función $\psi$ definida en (5.38) es no negativa. Entonces, el problema de optimización (5.37) se reduce a resolver

$$
\tau\left(x_{0}\right)=\frac{1}{r} \max _{x} h(x) \geq 0
$$

t.q. $\quad x \in \partial \mathcal{E}(c), \quad$ donde $c=V\left(x_{0}\right)$.

Expresando el programa anterior en términos de la condición necesaria de primer orden para un extremo, tenemos que existe $\left(x^{*}, \lambda^{*}\right) \in \mathbb{R}^{n} \times \mathbb{R}$, donde $x^{*}$ es un punto regular de la restricción y $\lambda^{*} \in \mathbb{R}$ es un multiplicador de Lagrange, tal que el Lagrangiano

$$
L_{\lambda^{*}}\left(x^{*}\right)=h\left(x^{*}\right)-\lambda^{*}(V(x)-c),
$$

para $c>0$ fija, satisface

$$
\nabla_{x} L_{\lambda^{*}}\left(x^{*}\right)=\nabla_{x} h\left(x^{*}\right)-\lambda^{*} \nabla_{x} V\left(x^{*}\right)=0
$$

En el caso que $\psi(5.38)$ sea no positiva, la versión negativa de (5.39), i.e. $\tau\left(x_{0}\right)=-\frac{1}{r} \min _{x} h(x) \geq$ 0 , debe ser considerada.

El procedimiento desarrollado en esta subsección será aplicado en las Secciones 5.2 y 6, para el caso de sistemas bilineales.

\subsection{Una clase de sistemas homogéneos}

Dado que tanto $U(\rho)(5.11)$ como $\mathcal{E}(c)$ (5.15) podrían no ser conjuntos simplemente (o ni siquiera) conexos, esto da lugar a problemas difíciles para la implementación del programa (5.21). A fin de garantizar una solución constructiva de (5.21), una condición suficiente podría ser que exista una función de Lyapunov homogénea $V(x)$ asociada al sistema (5.1) y que las funciones $g_{j}(x), j=1, \ldots, m$, también sean homogéneas. Si éste es el caso, el problema de optimización no sólo es factible sino ademés, el costo computacional implicado en resolverlo para cada valor del parámetro $c$ se ve reducido significativamente: El problema se resuelve solamente una vez. 
Teorema 5.1. Consideremos la familia uno-paramétrica de problemas de optimización (5.21). Supongamos que (5.1) admite una función de Lyapunov homogénea $V(x)$ de grado par $d y$ $g_{j}(x), j=1, \ldots, m$, son todas funciones homogéneas de grados iguales a $q$. Denotemos con $k$ $(=d+q-1)$ el grado de $h_{j}(x)=L_{g_{j}} V(x), j=1, \ldots, m, y$ definamos a $s$ como un número dado por $s h=d$. Supongamos además que $x^{*} \in \mathbb{R}^{n}$ es una solución óptima de (5.21) que satisface $V\left(x^{*}\right)=1 / s$. tal que ésta es un punto regular de la restricción y $g\left(x^{*}\right)$ tiene rango máximo. Entonces, para cualquier $x \in \mathbb{R}^{n}$, tenemos

$$
\tau(x)=\frac{1}{r} V(x)^{1 / s}\left\|\left(h_{1}^{p-1}\left(x^{*}\right), \ldots, h_{m}^{p-1}\left(x^{*}\right)\right) D h\left(x^{*}\right) g\left(x^{*}\right)\right\|_{p}^{1 /(p+1)} .
$$

Prueba. Elijamos $0 \neq x_{0} \in \mathbb{R}^{n}$, hagamos $c=V\left(x_{0}\right)(>0)$ y sea $x^{*} \in \mathbb{R}^{n}$ una solución óptima del programa propio (5.21). Entonces, por hipótesis y basaclos en el método de multiplicadores de Lagrange, tenemos que $x^{*}$ es un punto regular de la restricción y existe $\lambda^{*} \in \mathbb{R}$ tal que el siguiente Lagrangiano

$$
L_{\lambda^{*}}^{s}\left(x^{*}\right)=\frac{1}{r^{s}}\left\|h\left(x^{*}\right)\right\|_{p}^{s}-\lambda^{*}\left(V\left(x^{*}\right)-c\right)
$$

para $c>0$ fija, satisface

$$
\nabla_{x} L_{\lambda^{*}}^{s}\left(x^{*}\right)=\frac{s \tau^{s}}{p\left\|^{*}\right\|_{p}^{p}} \nabla_{y}\left\|y^{*}\right\|_{p}^{p} D h\left(x^{*}\right)-\lambda^{*} \nabla_{x} V\left(x^{*}\right)=0
$$

donde $y^{*}=h\left(x^{*}\right)$. Obviamente, $\|y\|_{p}^{p}$ es una función definida positiva, convexa y homogénea de grado $p$ (en la variable $y$ ). Además, $\left\|h\left(x^{*}\right)\right\|_{p}^{p} \neq 0$, pues $\tau\left(x^{*}\right)>0$ (Proposición $3.2(b)$ ). Entonces, por la homogeneidad de $V(x)$ y $h(x), \nabla_{x} L_{\lambda^{+}}^{s}(x)$ es una función homogénea de grado $d-1$. Por hipótesis, $g\left(x^{*}\right)$ tiene rango máximo. Así, multipliquemos por la derecha ambos lados de (5.44) por $g\left(x^{*}\right)$ para obtener

$$
\nabla_{x} L_{\lambda^{*}}^{s} g=\frac{s \tau^{s}}{\left\|y^{*}\right\|_{p}^{p}}\left(h_{1}^{p-1}, \ldots, h_{m}^{p-1}\right) D h g-\lambda^{*} h^{\top}=0
$$

Entonces, de la expresión anterior y dado que $\left\|u\left(x^{*}\right)\right\|_{p}=\left\|\frac{1}{r\left(x^{*}\right)} h^{\top}\left(x^{*}\right)\right\|_{p}=r$, tenemos

$$
\lambda^{*}=\frac{s \tau^{s-1}}{r\left\|y^{*}\right\|_{p}^{p}}\left\|\left(h_{1}^{p-1}, \ldots, h_{m}^{p-1}\right) D h g\right\|_{p}
$$

Consideremos nuevamente (5.44) y multipliquemos por la derecha ambos lados por $x^{*}$ para obtener

$$
\nabla_{x} L_{\lambda^{*}}^{s} x^{*}=\frac{s \tau^{s}}{p\left\|y^{*}\right\|_{p}^{p}} \nabla_{x}\left\|y^{*}\right\|_{p}^{p} x^{*}-\lambda^{*} \nabla V\left(x^{*}\right) x^{*}=0
$$

Observemos que $V(x)$ y $\|h(x)\|_{p}^{p}$ son funciones homogéneas de grados $d$ y $p h$, respectivamente. Entonces, por (la fórmula de Euler) homogeneidad, tenemos que $\nabla_{x} V(x) x=d V(x)$ y $\nabla_{x}\|h(x)\|_{p}^{p} x=p k\|h(x)\|_{p}^{p}$. Entonces, de (5.47) y debido a la Proposición $\left.3.2(b)\left(\left\|y^{*}\right\|_{p}^{p}\right) \neq 0\right)$. obtenemos

$$
\nabla_{x} L_{\lambda}^{s} \cdot x^{*}=\frac{s \tau^{s}}{\left\|y^{*}\right\|_{p}^{p}} l i y^{*} \|_{p}^{p}-d \lambda^{*} V\left(x^{*}\right)=d\left(\tau^{s}-\lambda^{*} V\left(x^{*}\right)\right)=0
$$

Por lo tanto, tenemos

$$
\tau^{s}(x)=\lambda^{*} V(x), \text { para cualquier } x \in \partial \mathcal{E}(c) \text {. }
$$


Por otra parte, usando la homogeneidad, tenemos que $\Sigma=\left\{x \in \mathbb{R}^{n}: x=\eta x^{*}, \eta>0\right\}$ es una línea de puntos críticos de $L_{\lambda *}^{s}$, es un conjunto de puntos regulares de la restricción, y además, $g \mid \Sigma$ tiene rango máximo. Además, basados en la prueba de la Proposición 3.2 (b), se sigue que $\Sigma$ es también un conjunto de máximos para el programa de optimización (5.21). Luego cualquier óptimo $x^{*}$ puede representarse como $x^{*}=\eta x_{0}^{*}$, donde, sin pérdida de generalidad, $x_{0}^{*}$ denota un óptino sujeto a $V\left(x_{0}^{*}\right)=1 / \mathrm{s}$. Por lo tanto, a fin de definir $\tau(x)$ dada en (5.49) en todo $\mathbb{R}^{22}$, procedenos como sigue: Dado un punto arbitrario $x_{0}$, hagamos $c=V\left(x_{0}\right)$ y sea $x^{*} \in \partial \mathcal{E}(c)$ la solución óptima comespondiente. En vista de que $x^{*}=\eta x_{0}^{*}$. tenemos $c=V\left(x^{*}\right)=\eta^{d} / s, y$ substituyendo esta expresión en (5.49), obtenemos $\eta^{d}(\tau)=s \tau^{s} / \lambda^{*}$. Así.

$$
x^{*}(\tau)=\left(\frac{s}{\lambda^{*}} \tau^{s}\right)^{1 / d} x_{0}^{*}
$$

Renumbremos $x_{0}^{*}$ con $x^{*}$. Entonces, si reemplazamos $x^{*} \operatorname{con} x^{*}(\tau)$ en (5.46), después de algunos cálculos algebraicos directos, obtenemos

$$
\lambda^{*}=\frac{s}{r^{s}}\left\|\left(h_{1}^{p-1}\left(x^{*}\right), \ldots, h_{m}^{p-1}\left(x^{*}\right)\right) D h\left(x^{*}\right) g\left(x^{*}\right)\right\|_{p}^{s /(p+1)}
$$

Finahmente, si sustituimos la expresión anterior en (5.49) obtenemos (5.42), que está definido en $R^{n}$.

Observación 7. Vale la pena mencionar que $\tau(x)$ dada en $(5.42)$ es también una función de Lyapunov propia (i.e., es definida positiva, radialmente no acotada y $d \tau / d t<0$ para toda $x \in \mathbb{R}^{n} \backslash\{0\}$ ), a condición de que la hipótesis H2 se satisfaga, y además, es no Lipschitsz en el origen.

Teorema 5.2. Consideremos el sistema afin (5.1) para el cual se satisficen las hipótesis H1 y H2. Si además suponemos las hipótesis del teorema anterior, tenemos que el control $u^{\times}(x)$ (5.23), donde $u_{\tau}(x)$ está dado por

$$
u_{\tau}(x)=-\frac{r}{\beta^{*} V^{1 / s}(x)}\left(L_{g} V(x)\right)^{\top},
$$

satisface $\left\|u^{\times}(x)\right\|_{p} \leq r$, con $\tau^{\times}>0$ arbitraria, estabiliza global asintóticamente el sisteina (5.1)(5.23), donde $\beta^{*}=\left\|\left(h_{1}^{p-1}\left(x^{*}\right), \ldots, h_{m}^{p-1}\left(x^{*}\right)\right) D h\left(x^{*}\right) g\left(x^{*}\right)\right\|_{p}^{1 /(p+1)}$ y $x^{*}$ es una solución óptima que satisface $V\left(x^{*}\right)=1 / \mathrm{s}$. Adcmás, tenemos una derivada global acotada de la entrada, i.e. para toda $x \in \mathbb{R}^{n}$, existe $\kappa>0$, tal que $\left\|d u^{\times} / d t\right\|_{p} \leq \kappa$, siempre que $L_{f} V(x) \in \mathcal{O}\left(\|x\|_{p}^{d}\right)$, $\|D h(x) f(x)\|_{p} \in \mathcal{O}\left(\|x\|_{p}^{k}\right) y$, o bien $g(x)=B$-una matriz $n \times m$ constante, o bien $g_{j}(x)=D_{j} x$. $j=1, \ldots, m$. El valor del parámetro $\kappa$ depende de las constantes asociadas a los órdenes, de la cota del control $r$ y del parámetro $\tau^{x}$.

Prueba. El resultado se sigue de la Proposición 3.1 y del teorema anterior. Sólo la afirmación sobre la cota de $\left\|d u^{\times} / d t\right\|_{p}$ requiere probarse. Denotemos $\tau(x)=\alpha V^{1 / s}(x)$, donde $\alpha=\beta^{*} / r$. Entonces, de $u_{\tau}(x)$ dado en (5.52), (5.32) y algunos cálculos algebraicos, obtenemos

$$
\begin{aligned}
\dot{u}_{\tau} & =\frac{1}{\tau}\left(\frac{\alpha^{s}}{s \tau^{s}}\left(L_{f} V+L_{g} V u_{\tau}\right) h-D h\left(f+g u_{\tau}\right)\right) \\
& =\left(\frac{\alpha^{s}}{s \tau^{s-1}}\left\|u_{\tau}\right\|_{2}^{2}-\frac{\alpha^{s}}{s \tau^{s}} L_{f} V\right) u_{\tau}-\frac{1}{\tau} D h f-\frac{1}{\tau}\left(g^{\top} \nabla_{x x} V g+D g^{\top}\left(\nabla_{x} V\right)^{\top} g\right) u_{\tau} .
\end{aligned}
$$


De la expresión anterior, y recordando que para toda $x \in \mathbb{R}^{n},\left\|u_{\tau}(x)\right\|_{p} \leq r$, obtenemos

$$
\left\|i_{\tau}\right\|_{p} \leq \frac{r^{3} \alpha^{s}}{s \tau^{s-1}}-\frac{r}{s V} L_{f} V+\frac{1}{\tau}\|D h f\|_{p}+\frac{r}{\tau}\left\|g^{\top} \nabla_{x x} V g+D g^{\top}\left(\nabla_{x} V\right)^{\top} g\right\|_{p} .
$$

Dado que $\tau(x)$ es una función propia, tenemos que el lado derecho de la expresión anterior está acotado en $\mathrm{R}^{n} \backslash \mathcal{E}\left(\tau^{\times}\right)$, para $\tau^{\times}>0$ arbitraria, si en los últimos tres términos, los órdenes de los denominadores (como funciones de $\tau$ ) son mayores o iguales a los correspondientes de los numeradores. Así, observando que $\tau(x)$ tiene (grado) orden $k(=d+q-1)$, obtenemos que $L_{f} V(x) \in \mathcal{O}\left(\|x\|_{p}^{d}\right)$ y $\|D h(x) f(x)\|_{p} \in \mathcal{O}\left(\|x\|_{p}^{k}\right)$ son condiciones de acotabilidad para el segundo y tercer términos de (5.53). Finalmente, el último término contiene una p-norma inducida $x$ dependiente, aplicada a una matriz $m \times m$ con entradas homogéneas de grado par, $d+2 q-2$. Por lo tanto, debemos tener $d+q-1 \geq d+2 q-2$, i.e. $q \leq 1$, para obtener el resultado deseado. Sin embargo, la suavidad de $g(x)$ sobre todo $\mathbb{R}^{n}$ (específicamente en $x=0$ ) restringe los valores de $q$ bien sea a 0 o a 1, y además $g(x)$ admite sólo dos posibilidades: o bien $g(x)=B$ -una matriz $n \times m$ constante, o bien $g_{j}(x)=D_{j} x$, donde $D_{j}$ son matrices $n \times n$ constantes, $j=1, \ldots, m$. Por otra parte, en $\mathcal{E}\left(\tau^{\times}\right)$, el lado derecho de (5.53) es obviamente acotado. De donde, recordando (5.23), tenemos que para toda $x \in \mathbb{R}^{n}$, $\left\|d u^{\times} / d t\right\|_{p} \leq \kappa$, donde $\kappa>0$ es un parámetro que depende de las contantes de los órdenes asociados, la cota del control $r$ y el valor del parámetro $\tau^{\times}$.

La clase de sistemas homogéneos considerada arriba incluye aquellos sistemas para los cuales $g(x)=B$ y que son estabilizables global asintóticamente mediante una función cuadrática de Lyapunov (los sistemas lineales incluso) y los sistemas bilineales homogéneos. En particular, para los sistemas lineales y bilineales, dado que sus dinámicas libres son lineales (i.e. $f(x)=A x)$, el resultado de derivadas de las entradas globalmente acotadas se garantiza en vista del teorema anterior. Este hecho será mostrado explícitamente en las Proposiciones 5.3 y 5.6.

\subsubsection{Sistemas estabilizables global asintóticamente mediante control lineal}

Consideremos la clase de los sistemas afines

$$
\dot{x}=f(x)+B u
$$

donde, sin pérdida de generalidad, suponemos que $B$ tiene rango máximo.

El método propuesto es particularmente útil para cualquier sistema (5.54) que puede estabilizarse global asintóticamente mediante una retroalimentación lineal, $u_{L}(x)=-\left(L_{B} V(x)\right)^{\top}=$ $-K x$, y función cuadrática de Lyapunov $V(x)=1 / 2 x^{\top} P x$, donde $P$ es una matriz $n \times n$ definida positiva y simétrica. Para tal sistema el problema de optimización es fácil de implementar, y considerando a las hipótesis $\mathrm{H} 1$ y H2, se puede diseñar una función RCA globalmente estabilizante. Hay en la literatura varias condiciones suficientes para la existencia de controles lineales, $c f$. por ejemplo [3]-[6]-[38]-[68].

Como hemos remarcado anteriormente, el problema de restricción del control $\|u(x)\|_{p} \leq r$ puede ser resuelto mediante el método de multiplicadores de Lagrange. En el presente caso, ese 
problema se reduce a encontrar una función $c=c(\tau)$ como la solución de la frontera elipsoidal (una relación)

$$
\partial \mathcal{E}(\tau)=\left\{x \in \mathbb{R}^{n}: V(x)=\frac{1}{2} x^{\top} P x=c(\tau)\right\}
$$

donde $P=P^{\top}>0$, sujeto a $\|u(x)\|_{p}=\left\|-\frac{1}{\tau} u_{L}(x)\right\|_{p} \leq r$. Resolviendo este problema de optimización, el parámetro $\tau$ se convierte en una función de los estados $(\tau=\tau(x))$.

Proposición 5.3. Supongamos que el sistema afín (5.54) satistace la hipótesis H1 con una función cuadrática de Lyapunov $V(x)$ dada en (5.55) y además, sin pérdida de generalidad, que $B$ tiene rango máximo. Si la hipótesis $\mathrm{H} 2$ es válida, entonces el control $u^{\times}(x)(5.23)$, donde $u_{\tau}(x)$ está dado por

$$
u_{-}(x)=-\frac{r}{\beta^{*} \sqrt{x^{\top} P x}} B^{\top} P x
$$

satisface $\left\|u^{\times}(x)\right\|_{p} \leq r, \operatorname{con} \tau^{\times}>0$ arbitraria, estabiliza global asintóticamente el sistema (5.54)(5.23), donde $\beta^{*}=\left\|\left(h_{1}^{p-1}\left(x^{*}\right), \ldots, h_{m}^{p-1}\left(x^{*}\right)\right) B^{\top} P B\right\|_{p}^{1 /(p+1)}$ y $x^{*}$ es una solución óptima que satisface $x^{* \top} P x^{*}=1$. Además, si $f(x)$ es una función globalmente Lipschitz en el origen (i.e. existe $L>0$ tal que para toda $x \in \mathbb{R}^{n}$, $\left.\|f(x)\|_{2} \leq L\|x\|_{2}\right)$, entonces tenemos $\left\|d u^{\times} / d t\right\|_{p} \leq \kappa$, para toda $x \in \mathbb{R}^{n}$, donde $r>0$ está definido en términos de las matrices $B$ y $P$. la constante de Lipschitz $L$, la cota del control $r$ y el parámetro $\tau^{\times}$.

Prueba. Observemos que $g(x)=B$ tiene rango máximo y $h(x)=B^{\top} P x$. Además, como $\nabla_{x} V(x) \neq 0$, para toda $x \neq 0$, cualquier extremo $x^{*}$ es un punto regular de la restricción. Hagamos $s=2$, pues $d=2$ y $h=1$. Entonces, basados en el Teorema 5.2, $\tau(x)$ está dada por (5.42) y es admisible (debido a la Proposición 3.2 (a) o (b)), entonces el resultado de estabilidad global se sigue.

Por otra parte, denotemos $\tau(x)=\alpha \sqrt{x^{\top} P x}$, donde $\alpha=\beta^{*} / r$. Así, de $u_{\tau}(x)$ dado en $(5.56)$ $y$ algunas manipulaciones algebraicas, obtenemos

$$
\begin{aligned}
i_{\tau} & =\frac{1}{\tau}\left(\frac{\alpha^{2}}{\tau^{2}}\left(x^{\top} P f(x)+x^{\top} P B u_{\tau}\right) B^{\top} P x-\left(B^{\top} P f(x)+\left(B^{\top} P B\right) u_{\tau}\right)\right) \\
& =\frac{1}{\tau}\left(\alpha^{2}\left(\left\|u_{\tau}\right\|_{2}^{2}-\frac{1}{\tau} x^{\top} \operatorname{Pf}(x)\right) u_{\tau}-\left(B^{\top} P f(x)+\left(B^{\top} P B\right) u_{\tau}\right)\right) .
\end{aligned}
$$

De la expresión anterior, y recordando que para toda $x \in \mathbb{R}^{n},\|f(x)\|_{2} \leq L\|x\|_{2}$ y $\left\|u_{\tau}(x)\right\|_{p} \leq r$, finalmente obtenemos

$$
\left\|u^{\times}\right\|_{p} \leq \kappa:=r\left(L\left(\frac{\lambda_{\max }(P)}{\lambda_{\min }(P)}+\frac{\left\|B^{\top} P\right\|_{2}}{\beta^{*} \lambda_{\min }\left(P^{1 / 2}\right)}\right)+\frac{1}{\tau^{\times}}\left(\beta^{* 2}+\left\|B^{\top} P B\right\|_{p}\right)\right),
$$

para toda $x \in \mathbb{R}^{n}$.

Observación 8. La cota $\kappa$ en (5.57) admite un significado geométrico en términos de la curvatura del elipsoide $\mathcal{E}(\tau)(5.55)$. En efecto, de (5.57) observemos que

$$
\lambda_{\text {max }}(P) / \lambda_{\min }(P)=\left(\zeta_{\max } / \zeta_{\text {min }}\right)^{2},
$$

donde $\zeta_{\min }$ y $\zeta_{\max }$, corresponden a las longitudes míninia y máxima de los semiejes de $\left.\partial \mathcal{E}(\tau)\right|_{c=1 / 2}$. Así, mientras más cercano sea el elipsoide a una bola, mínimo será el valor obtenido para $k$. 
Ejemplo 5.4. Consideremos el problema de estabilizar la velocidad angular de un vehículo espacial rígido. Las ecuaciones dinámicas para el problema no simétrico son [6]

$$
\begin{aligned}
& \dot{x}_{1}=I_{23} x_{2} x_{3}+\frac{u_{1}}{I_{1}} \\
& \dot{x}_{2}=I_{31} x_{3} x_{1}+\frac{u_{2}}{I_{2}} \\
& \dot{x}_{3}=I_{12} x_{1} x_{2}+\frac{u_{3}}{I_{3}}
\end{aligned}
$$

donde $I_{23}=\left(I_{2}-I_{3}\right) / I_{1}, I_{31}=\left(I_{3}-I_{1}\right) / I_{2}, I_{12}=\left(I_{1}-I_{2}\right) / I_{3}$ con $I_{i} \neq 0, j=1,2.3$, son los momentos principales de inercia del vehículo espacial rígido. Este sistema satisface las hipótesis $\mathrm{H} 1$ y $\mathrm{H} 2$. El siguiente control lineal global (no acotado)

$$
u_{L}(x)=-\left(L_{B} V(x)\right)^{\top}=-B^{\top} P x=-x,
$$

puede ser propuesto para obtener la EGA del sistema (5.59). Para probar esto último, se eligió la siguiente función cuadrática de Lyapunov

$$
V(x)=\frac{1}{2}\left(I_{1} x_{1}^{2}+I_{2} x_{2}^{2}+I_{3} x_{3}^{2}\right)
$$

Por otra parte, $\tau(x)(5.42)$ está dada por

$$
\tau(x)=\frac{\beta^{*}}{r} \sqrt{V(x)}
$$

donde $\beta^{*}=\left\|\left(\left(x_{1}^{*}\right)^{p-1},\left(x_{2}^{*}\right)^{p-1},\left(x_{3}^{*}\right)^{p-1}\right) B\right\|_{p}^{1 /(p+1)}=1 / \sqrt{I_{m}}$, e $I_{m}=\min _{i}\left\{I_{i}\right\}$. Por lo tanto, la función RCA global está dada por

$$
u(x)=\frac{1}{\tau(x)} u_{L}(x)
$$

Para propósitos de simulación, los valores de los parámetros se ajustaron a $I_{1}=1, I_{2}=1.5 \mathrm{e}$ $I_{3}=2$ (así $\beta^{*}=1$ ), la cota del control $r=1$ y la condición inicial $\bar{x}=(4,1,1)$. En la Figura 1 , puede verse la evolución de la función RCA (5.63) (arriba) y la de los estados a lazo cerrado (abajo).

\section{Sistemas lineales}

Consideremos el sistema lineal

$$
\dot{x}=A x+B u
$$

donde $A$ es una matriz $n \times n$ que satisface la hipótesis $\mathrm{H} 1$ y $B$ es una matriz $n \times m$. En este caso, la hipótesis H1 puede refrasearse en términos de funciones cuadráticas de Lyapunov, como sigue.

Hipótesis H1'. Supongamos que existe una matriz definida positiva y simétrica $P\left(=P^{\top}>0\right)$, que es solución de la ecuación de Lyapunov

$$
A^{\top} P+P A=-Q
$$


donde $Q$ es un matriz semidefinida positiva $(Q \geq 0)$ y $(\cdot)^{\top}$ denota transposición. Entonces, la función cuadrática

$$
V(x)=\frac{1}{2} x^{\top} P x
$$

define una función de Lyapunov para el sistema a lazo abierto (5.64).

De la Proposición 5.3, si en añadidura se satisface la hipótesis H2, la EGA del sistema (5.64) con control acotado estará garantizada.

Corolario 5.5. Consicleremos el sistema lineal (5.64) que satisface las hipótesis H1'y H2. y supongamos además. sin pérdida de generalidad. que $B$ tiene rango máximo. Entonces. la función de control $u^{\times}(x)$ (5.23) donde $u_{\tau}$ está dado en (5.56) satisface $\left\|u^{\times}(x)\right\|_{p} \leq r, y$ estabiliza global asintóticamente el sistema a lazo cerrado (5.64)-(5.23). Además. para toda $x \in \mathbb{R}^{n} .\left\|d u^{\times} / d t\right\|_{p} \leq \kappa$, donde $\kappa>0$ está dado en (5.57).

5.5.2 Sistemas bilineales homogéneos: $f(x)=A x$ y $g_{j}(x)=D_{j} x, j=1, \ldots, m$

Consideremos el caso bilineal homogéneo de (5.1)

$$
\dot{x}=A x+\sum_{j=1}^{m} u_{j} D_{j} x,
$$

donde $A$ es una matriz $n \times n$ que satisface la hipótesis $\mathrm{H} 1^{\prime}$ y $D_{j}, j=1, \ldots, m$, son matrices $n \times n$.

Bajo la hipótesis adicional $\mathrm{H} 2$, el control $u=\left(u_{1}, \ldots, u_{m}\right)$ con

$$
u_{j}(x)=-\frac{1}{\tau} h_{j}(x)=-\frac{1}{2 \tau} x^{\top}\left(P D_{j}+D_{j}^{\top} P\right) x, \quad j=1, \ldots, m
$$

estabiliza global asintóticamente al sistema (5.67), para $\tau>0$ arbitraria.

Basados en el Teorema 5.2, existe una función cuadrática de Lyapunov $V(x)$ dada por (5.66) tal que se puede diseñar un estabilizador global acotado y suave para la clase de sistemas bilineales homogéneos (5.67).

Proposición 5.6. Consideremos el sistema bilineal homogéneo (5.67) para el cual se satisface la hipótesis H1' con una función cuadrática de Lyapunov $V(x)$ dada en (5.55). Si además, se satisface la hipótesis $\mathrm{H} 2$, entonces el control $u^{\times}(x)(5.23)$, donde $u_{\tau}=\left(u_{\tau_{1}}, \ldots, u_{\tau_{n}}\right)$ está dado por

$$
u_{\tau_{j}}(x)=-\frac{r}{\beta^{*} x^{\top} P x} x^{\top}\left(P D_{j}+D_{j}^{\top} P\right) x, \quad j=1, \ldots, m,
$$

satisface $\left\|u^{\times}(x)\right\|_{p} \leq r$, con $\tau^{\times}>0$ arbitraria, estabiliza global asintóticamente el sistema (5.67)-(5.23), donde $\beta^{*}=\left\|\left(h_{1}^{p-1}\left(x^{*}\right), \ldots, h_{m}^{p-1}\left(x^{*}\right)\right) D h\left(x^{*}\right) g\left(x^{*}\right)\right\|_{p^{1 /(p+1)}}^{y} x^{*}$ es una solución óptima tal que $x^{* \top} P x^{*}=2$ y el conjunto $\left\{D_{1} x^{*}, \ldots, D_{m} x^{*}\right\}$ es linealmente independiente. Además, para toda $x \in \mathbb{R}^{n}$. $\left\|d u^{\times} / d t\right\|_{p} \leq \kappa$, para algún $\kappa_{i}>0$.

Prueba. Observernos que $g_{j}(x)=D_{j} x$. Además, como $\nabla_{x} V(x) \neq 0$, para toda $x \neq 0$, cualquier extremo $x^{*}$ es un punto regular de la restricción. Hagamos $s=1$, pues $d=2 y k=2$. Entonces. 
basados en el Teorema 5.2, $\tau(x)$ está dada por (5.42) y es admisible (debido a la Proposición 3.2 (b)), entonces el resultado de estabilización global se sigue.

Por otra parte, de $u_{\tau}(x)$ (5.69) y (5.65), la expresión (5.53) se convierte en

$$
\left\|i_{\tau}\right\|_{p} \leq r^{2} \beta^{*}+\frac{r}{\beta^{*} x^{\top} P x}\left(x^{\top} Q x\right)+\frac{1}{\tau}\|D h A x\|_{p}+\frac{r}{\tau}\left\|g^{\top} P g+D g^{\top} P x g\right\|_{p} .
$$

Observando que el lado derecho de la expresión anterior está acotado en $\mathbb{R}^{n} \backslash \mathcal{E}\left(\tau^{\times}\right)$, para cualquier $\tau^{\times}>0$, el resultado se sigue del Teorema 5.2.

En el caso particular de un sistema bilineal con control escalar, y partiendo del problema de optimización para restricciones con control escalar (5.37), la solucion de la ecuación (5.41) se reduce a encontrar una raíz del polinomio

$$
p(\lambda)=\operatorname{det} H_{\lambda}
$$

donde

$$
H_{\lambda}:=\left(P D+D^{\top} P\right)-\lambda P \text {. }
$$

Si $\lambda=\lambda^{*}$ es la raíz requerida, considerando (5.41) y (5.37), se sigue que $c(\tau)=r \tau /\left|\lambda^{*}\right|$. Por lo tanto.

$$
\tau(x)=\frac{\left|\lambda^{*}\right|}{2 r} x^{\top} P x
$$

y la función RCA global está dada por (5.23) con

$$
u_{\tau}(x)=-\frac{r}{\left|\lambda^{*}\right|\left(x^{\top} P x\right)} x^{\top}\left(P D+D^{\top} P\right) x .
$$

El siguiente teorema proporciona solución a problemas de optimización convexos y no convexos (5.37) asociados al problema RCA para sistemas bilineales homogéneos con control escalar. Dependiendo de si la función $\psi(5.38)$ es no negativa o no positiva definida, las condiciones suficientes son expresadas en términos de $\lambda^{*}$ y de las demás raíces del polinomio $p(\lambda)(5.71)$. Debemos notar que, de la homogeneidad, si $\psi(c)>0(<0)$ para algún $c>0$, entonces $\psi$ es definita positiva (negativa).

Teorema 5.7. Supongamos que se satisfacen las hipótesis $\mathrm{H}_{1}^{\prime}$ y $\mathrm{H} 2$, y sea $\lambda^{*}=\max _{\lambda} \arg \{p(\lambda)=$ $0\}$. Entonces: (i) si tenemos el problema de optimización (5.39), entonces $\lambda^{*}$ es la única raíz positiva del polinomio $p(\lambda)$; o (ii) si tenemos la versión negativa de (5.39), entonces todas las raices del polinomio $p(\lambda)$ son negativas. En cualquier caso, la EGA del sistema bilineal homogéneo se obtiene mediante la función RCA $u(x)$ dada por (5.74).

Prueba.

Caso (i): Como es bien sabido [40], una condición suficiente (de segundo orden) para que $x^{*}$ sea un máximo del problema de optimización (5.39) consiste en

(a) la condición necesaria: existe $\lambda^{*} \in \mathbb{R}$ tal que

$$
x^{* \top} H_{\lambda^{*}}=x^{* \top}\left(\left(P D+D^{\top} P\right)-\lambda^{*} P\right)=0, \quad y
$$


(b) la matriz hessiana $H_{\lambda^{*}}$ (o pincel regular (regular pencil) de matrices (5.72)) sea semidefinida negativa en el subespacio

$$
M=\left\{z \in \mathbb{R}^{n}: x^{* \top} P z=0\right\}:=\operatorname{ker}\left(x^{* \top} P\right) .
$$

Entonces, si consideramos (5.75) y que $\tau(x)(5.39)$ es definida positiva, se sigue que $\lambda^{*}>0$. Se requieren los siguientes dos resultados, especializados a nuestro problema presente ( $c f$. por ejemplo [13]).

(A) (Teorema de Courant-Fischer) Existen $n$ valores característicos reales de $H_{\lambda}$, que podemos suponer ordenados: $\lambda_{1} \leq \ldots \leq \lambda_{n}$. Además, para cada $k(1 \leq k \leq n)$, el $k$-ésimo valor caracteristico $\lambda_{k}$ está dado como la mínima razón de la formas bilineales

$$
\lambda_{k}=\min _{x} \frac{x^{\top}\left(P D+D^{\top} P\right) x}{x^{\top} P x},
$$

siempre que el vector variable $x$ esté sujeto a $k-1$ relaciones lineales: existen $k-1$ vectores $z_{i}$ $(1 \leq i \leq k-1)\left(P\right.$-)ortonomales, i.e., $z_{i}^{\top} P z_{j}=\delta_{i j}$, que son ( $P$-)ortogonales a $x$, es decir

$$
x^{\top} P z_{1}=0, x^{\top} P z_{2}=0, \ldots, x^{\top} P z_{k-1}=0 .
$$

(B) Hay una transformación no singular, $x=T \xi$, que reduce las formas bilineales $x^{\top}(P D+$ $\left.D^{\top} P\right) x$ y $a^{\top} P x$ simultáneamente a una suma de cuadrados

$$
\xi^{\top} \operatorname{diag}\left(\lambda_{1}, \ldots, \lambda_{n}\right) \xi \quad \text { y } \xi^{\top} \xi .
$$

Entonces, $\lambda$ es un valor característico cel pincel (pencil) $H_{\lambda}$ sisi es una raíz del polinomio $p(\lambda)=\operatorname{det} H_{\lambda}$.

Afirmamos que si $\lambda^{*}$ es la única raíz positiva de $p(\lambda)$, entonces $H_{\lambda^{*}}$ es negativa semidefinida (la condición suficiente es válida). En efecto, como $\operatorname{dim} M=\operatorname{dim} \operatorname{ker}\left(x^{* \top} P\right)=n-1$, de $(A)$, se sigue que $k=n$. Basados en esto, si tomamos en cuenta $(B)$ y que, por hipótesis, $\lambda_{1}, \ldots, \lambda_{n-1}$ son raíces no positivas de $p(\lambda)$, entonces la matriz $H_{\lambda^{*}}$ (5.72) es semidefinida negativa en $M$. Además, $\lambda^{*}=\lambda_{n}>0$-el multiplicador de Lagrange asociado a $x^{*}$ - es una raíz del polinomio $p(\lambda)$. Por lo tanto, $\lambda_{1} \leq \ldots \leq \lambda_{n-1} \leq 0$ y $\lambda_{n}>0$ son todos los valores característicos de $H_{\lambda}$.

Caso (ii): Si $\psi(5.38)$ es no positiva, la versión negativa de (5.39) se da. Entonces, obtenemos la signiente condición necesaria para un extremo

$$
x^{* \top} H_{\nu^{*}}=0 .
$$

donde $H_{\nu^{*}}=\left(P D+D^{\top} P\right)+\nu^{*} P$. De esto y del hecho que $\tau(x)$ es definida positiva, se sigue que $\nu^{*}>0$. Denotemos con $\lambda^{*}:=-\nu^{*}$, para obtener que $\lambda^{*}<0$. Entonces, si consideramos que $p(\lambda)=\operatorname{det} H_{\lambda}(5.71)$, mediante un razonamiento análogo al del Caso (i), obtendremos que todas las raíces de $p(\lambda)$ deben ser negativas, con $\lambda^{*}$ la mayor de ellas.

Los resultados del teorema anterior se ilustran en el siguiente ejemplo para un sistema bilineal homogéneo en el plano. Ahí diseñanos una función RCA (5.74) que estabiliza global asintóticamente el sistema bilineal mediante la solución de un problema de optimización no convexo. 
Ejemplo 5.8. Consideremos el oscilador lineal controlado. Las ecuaciones dinámicas están dadas por el sistema bilineal homogéneo planar

$$
\left(\begin{array}{l}
\dot{x}_{1} \\
\dot{x}_{2}
\end{array}\right)=\left(\begin{array}{cc}
0 & 1 \\
-1 & -2 b
\end{array}\right)\left(\begin{array}{l}
x_{1} \\
x_{2}
\end{array}\right)+u\left(\begin{array}{cc}
0 & 0 \\
-1 & 0
\end{array}\right)\left(\begin{array}{l}
x_{1} \\
x_{2}
\end{array}\right), \quad|u| \leq r
$$

Para el sistema a lazo abierto $((5.81)$ con $u \equiv 0)$, el origen es un punto de equilibrio, que es asintóticamente estable para $b>0, y$ marginalmente estable para $b=0$. Estudiaremos el primer caso. pues la dinámica del segundo caso $(b=0)$ es análoga. Unas matrices $P$ y $Q$ que satisfacen (hipótesis $\mathrm{H} 1$ ') la ecuación (5.65) son, e.g.

$$
P=\alpha\left(\begin{array}{ll}
1 & \gamma \\
\gamma & 1
\end{array}\right)>0 \quad \text { y } \quad Q=2 \alpha\left(\begin{array}{cc}
\gamma & b \gamma \\
b \gamma & 2 b-\gamma
\end{array}\right)>0
$$

donde $\alpha>0$ y $0<\gamma<2 b /\left(1+b^{2}\right)(\leq 1)$. La hipótesis H2 también se satisface y $(0,0)$ es el único punto de equilibrio EGA tanto para el sistema a lazo abierto como para el de lazo cerrado dado por (5.81) y la retroalimentación (no acotada)

$$
u_{u n b}(x)=-h(x)=-L_{g} V(x)=\alpha\left(\gamma x_{1}^{2}+x_{1} x_{2}\right)
$$

En lugar de brindar una mejoría sobre el sistema a lazo abierto (EGA), la dinámica del sistema a lazo cerrado puede resultar muy insatisfactoria para valores de $b$ lejos de 1 (i.e. $0<b \ll 1$ ó $b \gg 1$ ). Fuera de alguna vecindad $N$ del origen, todas las trayectorias arriban muy rápido a la región donde la curva definida por $x_{2}=-\gamma x_{1}$ (que corresponde a $u(x)=0$ ) y la curva dada por $x_{2}=-\left(\alpha \gamma x_{1}^{2}+1\right) x_{1} /\left(\alpha x_{1}^{2}+2 b\right)$ (que corresponde a $d x_{2} / d t=0$ ) se hallan cerca, lo cual causa una tasa de convergencia muy pobre al origen (vea la Figura 3). Este comportamiento se debe a una respuesta errónea por parte del control, que tiene una magnitud grande cuando $x_{1}$ se halla lejos del origen, y una pequeña en el otro caso. Por otra parte, si se aplica el método de cliseño propuesto, este mal funcionamiento del control se ve significativamente mejorado. De (5.74), tenemos la ley de control acotada

$$
u\left(x_{1}, x_{2}\right)=\frac{2 r\left(\gamma x_{1}^{2}+x_{1} x_{2}\right)}{\lambda^{*}\left(x_{1}^{2}+2 \gamma x_{1} x_{2}+x_{2}^{2}\right)}
$$

donde $\lambda^{*}=1 / \sqrt{1-\gamma^{2}}$, es la única raíz positiva del polinomio $p(\lambda)=\operatorname{det} H_{\lambda}=\alpha^{2}\left(\lambda^{2}-1-\gamma^{2}\right)$ (5.71). Para propósitos de simulación, la condición inicial se tomó como $\bar{x}=(-8,8), b=0.05$, $\gamma=0.094763$ y $\alpha=r=1$. La Figura 2 muestra (arriba) la evolución del control no acotado $u_{u n b}(x)=-h(x)(5.83)$ y (debajo) la trayectoria correspondiente con pobre convergencia; mientras que, en la Figura 3, se muestra las gráficas de las curvas asintóticas mencionadas, causa del mal funcionamiento. Sin embargo, de mantener acotado el control resulta una mejor estrategia. La Figura 4 muestra (arriba) una retroalimentación saturada (RS) basada en (5.83) y (abajo) su dinámica a lazo cerrado asociada. Como puede observarse en la Figura 5. (arriba) la función RCA (5.84) es un control con buen desempeño, que proporciona (vea abajo) una mejor respuesta que la obtenida mediante el método con $\mathrm{RS}$. 


\subsection{Sistemas bilineales no homogéneos: $f(x)=A x$ y $g(x)=$ $D x+b$}

Consideremos el caso bilineal no homogéneo de (5.1) con control escalar

$$
\dot{x}=4 x+u(D x+b)
$$

donde A es una matriz $n \times n$ que satisface la hipótesis $\mathrm{H} 1^{\prime}$, $D$ es una matriz $n \times n$ y $b \in \mathbb{R}^{n}$.

Bajo la hipótesis adicional $\mathrm{H} 2$, la función de control escalar

$$
u(x)=-\frac{1}{\tau} h(x)=-\frac{1}{\tau}\left(\frac{1}{2} x^{\top}\left(P D+D^{\top} P\right) x+b^{\top} P x\right)
$$

es un estabilizador global del sistema (5.85), para $\tau>0$ arbitraria.

Si consideramos la restricción del control, $|u(x)| \leq r$, el parámetro $\tau$ estará definido funcionalmente en términos de los estados: $\tau=\tau(x)$. Con base en la Proposición 3.2 (c), tenemos que $\tau(x)$ es admisible. Como mencionamos al principio de la Sección 5 , las condición necesaria de primer orden para un extremo del problema de optimización (5.39) se reduce al de hallar. una función $c=c(\tau)$ definida por (5.55). Supongamos que la función $\psi$ (5.38) es no negativa. Entonces, existe $\left(x^{*}, \lambda^{*}\right) \in \mathbb{R}^{n} \times \mathbb{R}$, donde $x^{*}$ es un punto regular de la restricción $y \lambda^{*} \in \mathbb{R}$ es un multiplicador de Lagrange, tal que el Lagrangiano

$$
L_{\lambda^{*}}\left(x^{*}\right)=h\left(x^{*}\right)-\lambda^{*}(V(x)-c),
$$

para $c>0$ fija, satisface

$$
\nabla_{x} L_{\lambda} \cdot\left(x^{*}\right)=\nabla_{x} h\left(x^{*}\right)-\lambda^{*} \nabla_{x} V\left(x^{*}\right)=0 .
$$

Si definimos $\mu^{*}:=1 / \lambda^{*} \neq 0$, entonces (5.88) se vuelve equivalente a

$$
x^{* \top} P-\mu^{*}\left(x^{* \top}\left(P D+D^{\top} P\right)+b^{\top} P\right)=0 .
$$

Entonces,

$$
x^{*}=\mu^{*} H_{\mu}^{-1} P b,
$$

donde $H_{\mu^{*}}:=P-\mu^{*}\left(P D+D^{\top} P\right)$. Si tomamos en consideración (5.39), obtenemos que $\tau=\tau(\mu)$. está definida pol

$$
\tau(\mu)=\frac{1}{r}\left(\frac{1}{2} x^{* \top}\left(P D+D^{\top} P\right) x^{*}+b^{\top} P x^{*}\right),
$$

donde $x^{*}$ está dada por (5.90).

Mediante la sustitución de (5.90) en (5.55), obtenemos

$$
c(\tau)=\frac{1}{2} x^{* \top} P x^{*}
$$

De nuevo, si usamos (5.55) y la expresión anterior, el paránetro $\mu$ será una función de los estados, $\mu=\mu(x)$, definida implicitamente de la relación (frontela elipsoidal)

$$
x^{\top} P x-x^{* \top} P x^{*}=0 .
$$


Resolviendo (5.93) para cada $x \in \mathbb{R}^{n}$, obtenemos $\mu=\mu(x)$ y de $(5.91), \tau=\tau(x)$.

Lema 6.1. La expresión (5.90) está bien definida en un intervalo abierto máximo $I_{0} \subset \mathbb{R}$ que contiene a $\mu=0$. Además, en $I_{0}, x^{*}=0$ ssi $\mu=0$.

Prueba. En primer lugar, como $P>0$, el conjunto de valores característicos de $H_{\mu}, \mathcal{N}=$ $\left\{\mu\right.$ : det $\left.H_{\mu}=0\right\}$, es un subconjunto finito discreto de $\mathbb{R}[13]$. Así, la resolvente de $H_{\mu}$, $\mathcal{R}=\left\{\mu \in \mathbb{R}: \exists H_{\mu}^{-1}\right\}$, consiste de una unión finita ajena de intervalos abiertos. Además, existe un intervalo abierto máximo $I_{0} \subset \mathbb{R}$ que contiene a $\mu=0, I_{0}=\left(u_{-}, u_{+}\right)$, (posiblemente con $u_{-}=-\infty \circ u_{+}=\infty$, pero no ambos), tal que $I_{0} \subset \mathcal{R}$ (i.e., $\left.H_{\mu}^{-1}\right|_{\mu \in I_{0}}$ existe), porque $P>0$. Entonces si $\mu=0$, de (5.90), se sigue que $x^{*}=0$. Reciprocamente, si $x^{*}=\mu H_{\mu}^{-1} P b=0$, entonces o bien $\mu=0$ o bien $\mu \neq 0$ y $H_{\mu}^{-1} P b=0$. Si $\mu \neq 0$ y como $P>0$, para toda $\mu \in I_{0}$, tenemos que $H_{\mu}>0$; así este caso es equivalente a $H_{\mu}^{-1} P b=0$, es decir $P b \in \operatorname{ker} H_{\mu}^{-1}$. Entonces, dado que ker $H_{\mu}^{-1}=\{0\}$, para toda $\mu \in I_{0}$, y en vista que $P b \neq 0$, obtenemos una contradicción. Por lo tanto, $\mu=0$.

Lema 6.2. Si el polinomio $p(\mu)=\operatorname{det} H_{\mu}$ tiene una raíz positiva, entonces la ecuación (5.93) tiene al menos una solución positiva $\mu>0$, para cada $x \in \mathbb{R}^{n} \backslash\{0\}$.

Prueba. Reemplacemos (5.90) en (5.93), y definamos el polinomio (que depende de $x$ )

$$
\varphi(x, \mu)=\left(x^{\top} P x\right)[p(\mu)]^{2}-\mu^{2} b^{\top} P \operatorname{Adj}\left[H_{\mu}\right] P \operatorname{Adj}\left[H_{\mu}\right] P b .
$$

Supongamos que $x \neq 0$ (el caso de $x=0$, fue considerado en el lema anterior). Debido a que $P>0$, tenemos

- si $\mu=0, \varphi(x, 0)=\left(x^{\top} P x\right)[\operatorname{det} P\}^{2}>0$, para toda $x \in \mathbb{R}^{n} \backslash\{0\} ; \mathrm{y}$

- si $\mu \neq 0$, la ecuación $\operatorname{det} H_{1 / \mu}=0\left(=\operatorname{det} H_{\lambda}\right)$ tiene $n$ raíces reales [13]. Así, si ésta tiene una raíz positiva $\mu_{1}>0$, entonces $\varphi\left(x, \mu_{1}\right)<0$.

Con base en estos dos puntos, por continuidad de $\varphi(x, \mu)$, para cada $x \in \mathbb{R}^{n} \backslash\{0\}$, existe $\mu^{*}$, $0<\mu^{*}<\mu_{1}$, tal que $\varphi\left(x, \mu^{*}\right)=0$.

Con base en los dos lemas anteriores, el procedimiento desarrollado al principio de esta sección se ve validado y así, tenemos la siguiente proposición.

Proposición 6.3. Consideremos el sistema bilineal (5.85). Entonces, $\tau(x)$ es admisible y se obtiene de (5.91) y de la función definida implicitamente $\mu(x)$ (5.93). Además, si se satisfacen las hipótesis $\mathrm{H}_{1}$ ' y H2, entonces el control escalar $u^{\times}(x)$ dado en (5.23) con $u_{\tau}$ definido por

$$
u_{\tau}(x)=-\frac{1}{\tau(x)}\left(\frac{1}{2} x^{\top}\left(P D+D^{\top} P\right) x+b^{\top} P x\right)
$$

satisface $\left|u^{\times}(x)\right| \leq r, y$ estabiliza global asintóticamente el sistema a lazo cerrado (5.85)-(5.23), para $\tau^{\times}>0$ arbitraria.

La proposición anterior se ilustra en el siguiente ejemplo de un sistema bilineal no homogéneo planar. Ahí, se obtiene una función RCA definicla implicitamente mediante la solución de un problema de optimización no convexo. 
Ejemplo 6.4. Consideremos el siguiente sistema bilineal planar

$$
\left(\begin{array}{l}
\dot{x}_{1} \\
\dot{x}_{2}
\end{array}\right)=\left(\begin{array}{cc}
0 & 1 \\
0 & -1
\end{array}\right)\left(\begin{array}{l}
x_{1} \\
x_{2}
\end{array}\right)+u\left(\begin{array}{l}
0 \\
1
\end{array}\right)\left(x_{1}+1\right), \quad|u| \leq r .
$$

Las matrices $P$ y $Q$ que satisfacen la ecuación (5.65) son, e.g.

$$
P=\left(\begin{array}{cc}
\alpha-1 & \alpha-1 \\
\alpha-1 & \alpha
\end{array}\right)>0 \quad y \quad Q=\left(\begin{array}{ll}
0 & 0 \\
0 & 2
\end{array}\right) \geq 0
$$

donde $a>1$. En particular, para $\alpha=2$, obtenemos de $(5.90)-(5.91)-(5.92)$, que

$$
\tau(\mu)=\frac{2 \mu}{\mu\left(1-4 \mu^{2}\right)^{2}} \quad y \quad c(\mu)=\frac{\mu^{2}\left(1+4 \mu^{2}\right)}{\left(1-4 \mu^{2}\right)^{2}}
$$

Conde $\mu=\mu\left(x_{1}, x_{2}\right)$ está definida implicitamente (para cada $\left(x_{1}, x_{2}\right)$ fija, como la múnima solución positiva de) por la frontera elipsoidal (5.94)

$$
\varphi\left(x_{1}, x_{2}, \mu\right)=\left(1-4 \mu^{2}\right)^{2}\left(x_{1}^{2}+2 x_{1} x_{2}+2 x_{2}^{2}\right)-2 \mu^{2}\left(1+4 \mu^{2}\right)^{2}=0
$$

Vo us difícil verificar que $\tau(\mu)>0$ para $0<\mu<1 / 2$, que es cero sci $\mu=0\left(\operatorname{sisi}\left(x_{1}, x_{2}\right)=(0,0)\right.$. por el Lema 6.2). Entonces, de la composición $\tau\left(x_{1}, x_{2}\right):=\tau\left(\mu\left(x_{1}, x_{2}\right)\right)$. podemos mostrar que $\tau\left(x_{1}, x_{2}\right)$ es una función definida positiva cuyo dominio es todo $\mathbb{R}^{2}$. Además, tenemos que $d r / d \tau>0$, lo cual significa que $d \tau)$ es una función monótona creciento en $\tau$, y el elipsoide $\mathcal{E}(\tau)$ cubre todo el plano cuando $\tau \rightarrow \infty$. Por lo tanto, la función RCA

$$
u\left(x_{1}, x_{2}\right)=-\frac{1}{\tau\left(x_{1}, x_{2}\right)}\left(x_{1}+2 x_{2}\right)\left(x_{1}+1\right)
$$

es un estabilizador global para el sistema a lazo cerrado (5.96)-(5.100). En la Figura 6, se muestra la evolución de un control discontinuo basado en el articulo de Lin [34] (arriba), cuya expresión es

$$
u\left(x_{1}, x_{2}\right)=-\operatorname{signo}\left[\left(x_{1}+2 x_{2}\right)\left(x_{1}+1\right) \mid \min \left\{\left|\left(x_{1}+2 x_{2}\right)\left(x_{1}+1\right)\right|, 1\right\} .\right.
$$

y una trayectoria asociarla (abajo); mientras que en la Figura $\vec{r}$, puede verse (arriba) la evolnción de la función RCA (5.100) y (abajo) la trayectoria correspondiente. La condicion inicial es $\bar{x}=(100,-200)$. 

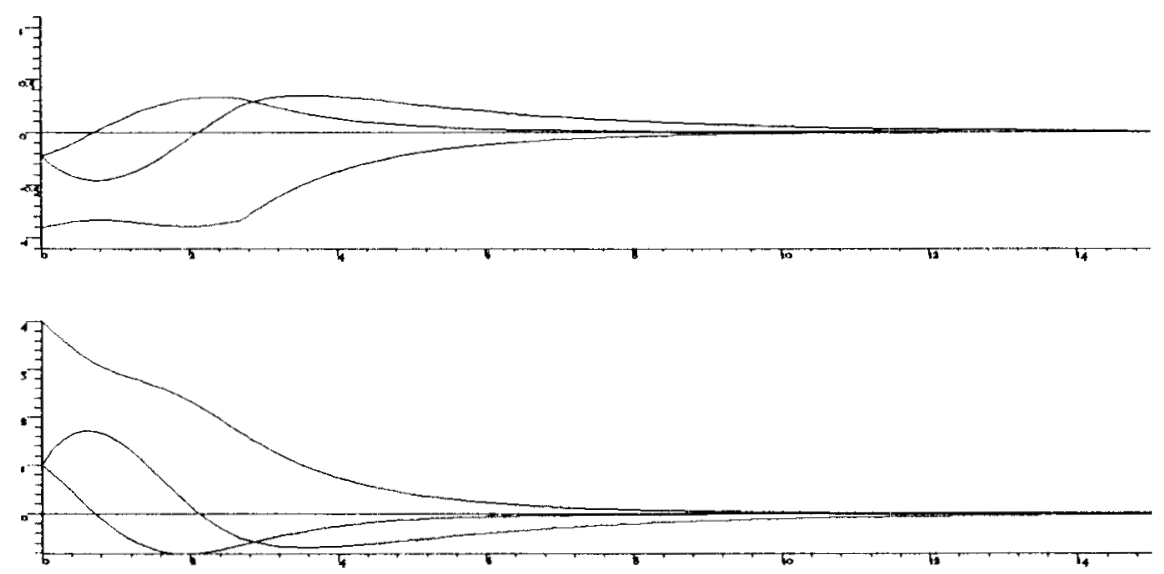

Figure 0.1: Ejemplo 5.4. Evolución de la función RCA (5.63) y abajo, los correspondientes estados a lazo cerrado, para la condición inicial $\bar{x}=(4,1,1)$. 

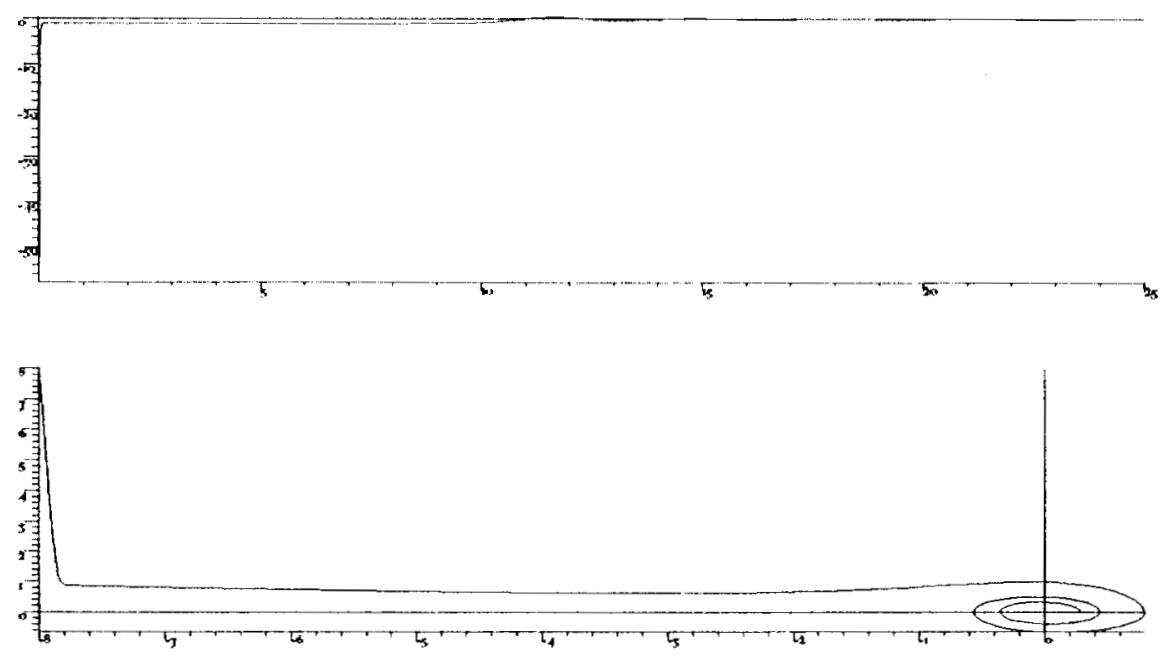

Figure 0.2: Ejemplo 5.8. Evolución del control no acotado (5.83) y abajo, la correspondiente trayectoria a lazo cerrado, para la condición inicial $\bar{x}=(-8,8)$.

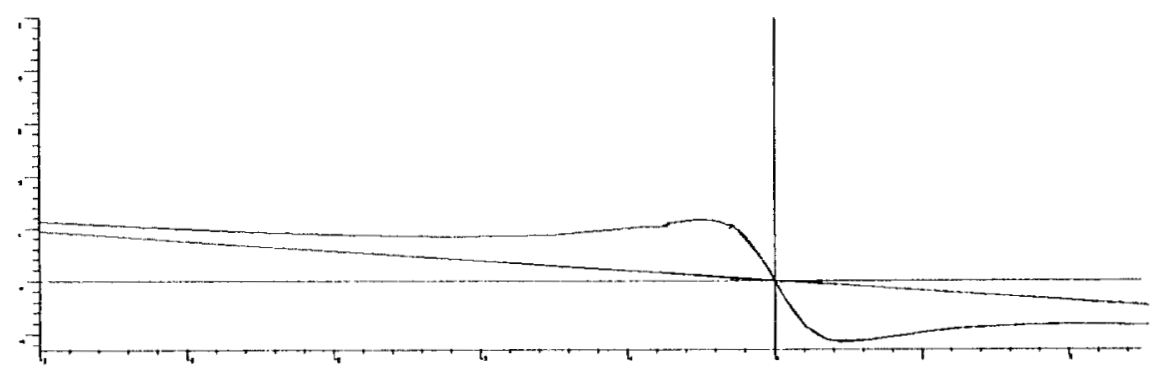

Figure 0.3: Ejemplo 5.8. Curvas sintóticas $x_{2}=-\left(\alpha \gamma x_{1}^{2}+1\right) x /\left(\alpha x_{1}^{2}+2 b\right)$ y $x_{2}=-\gamma x$, causa de la convergencia lenta al origen del control (5.83). 

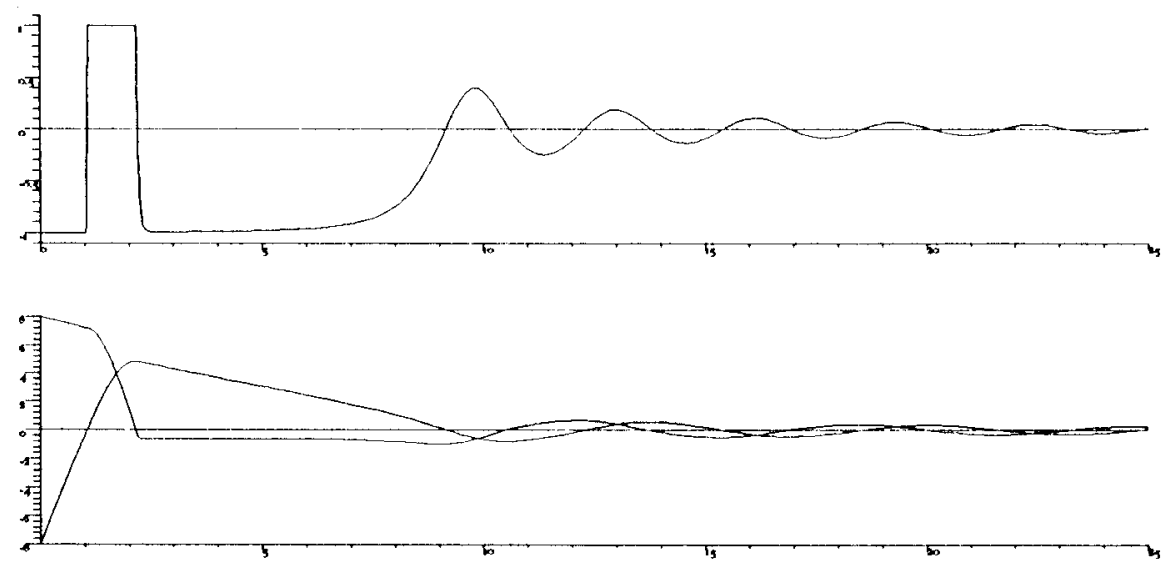

Figure 0.4: Ejemplo 5.8. Evolución de un control saturado basado en (5.83) y abajo, los correspondientes estados, para la condición inicial $\bar{x}=(-8,8)$.
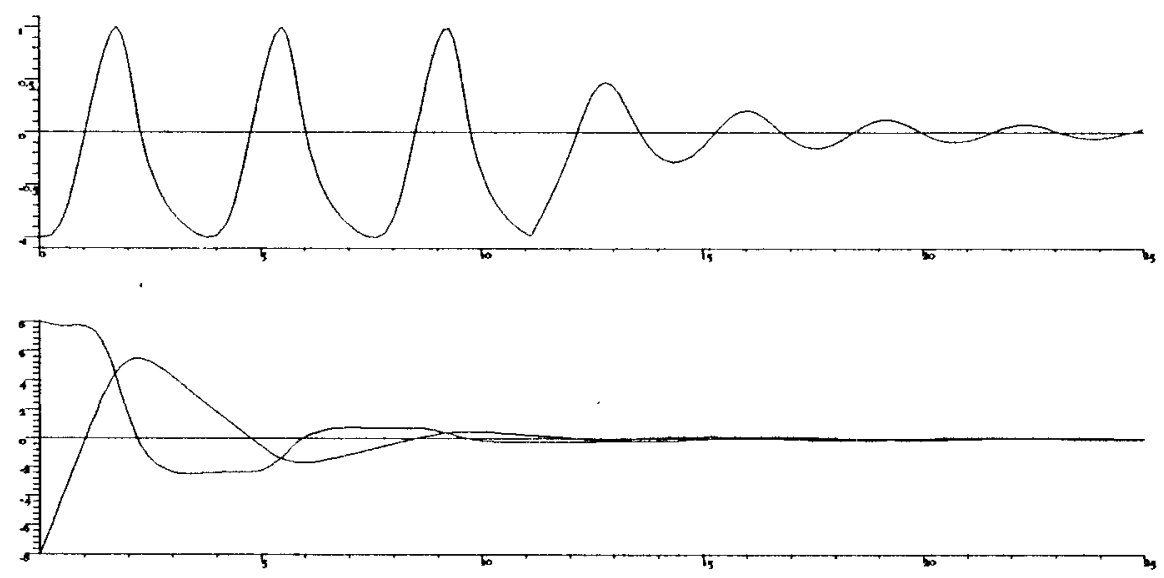

Figure 0.5: Ejemplo 5.8. Evolución de la función RCA (5.84) y abajo, los correspondientes estados, para la condición inicial $\bar{x}=(-8,8)$. 

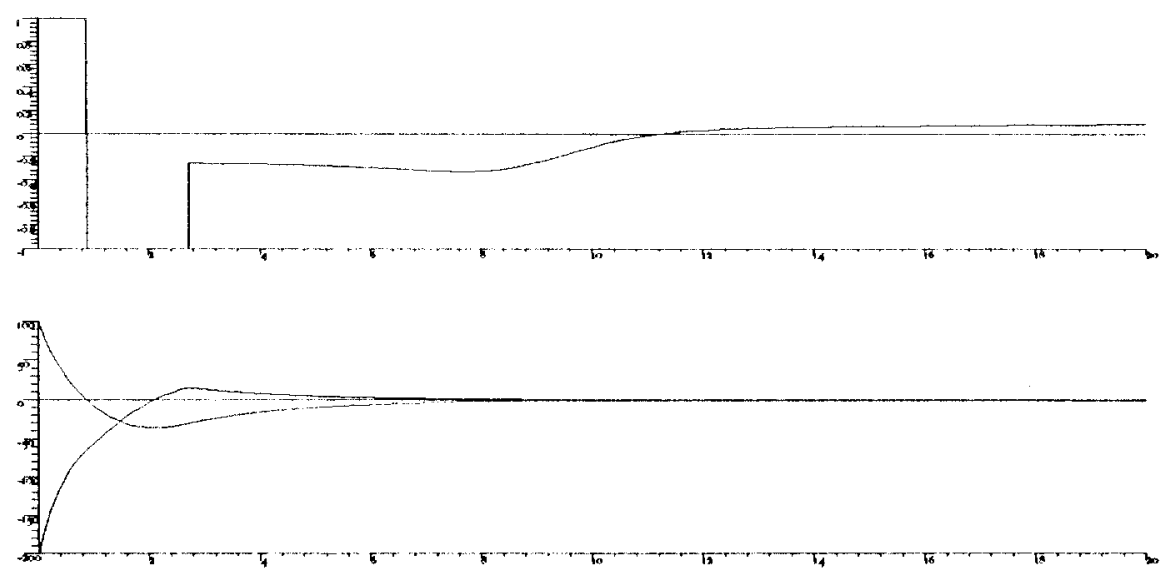

Figure 0.6: Ejemplo 6.4. Evolución del control basado en el artículo de Lin [33], y abajo, los correspondientes estados, para la condición inicial $\bar{x}=(100,-200)$.
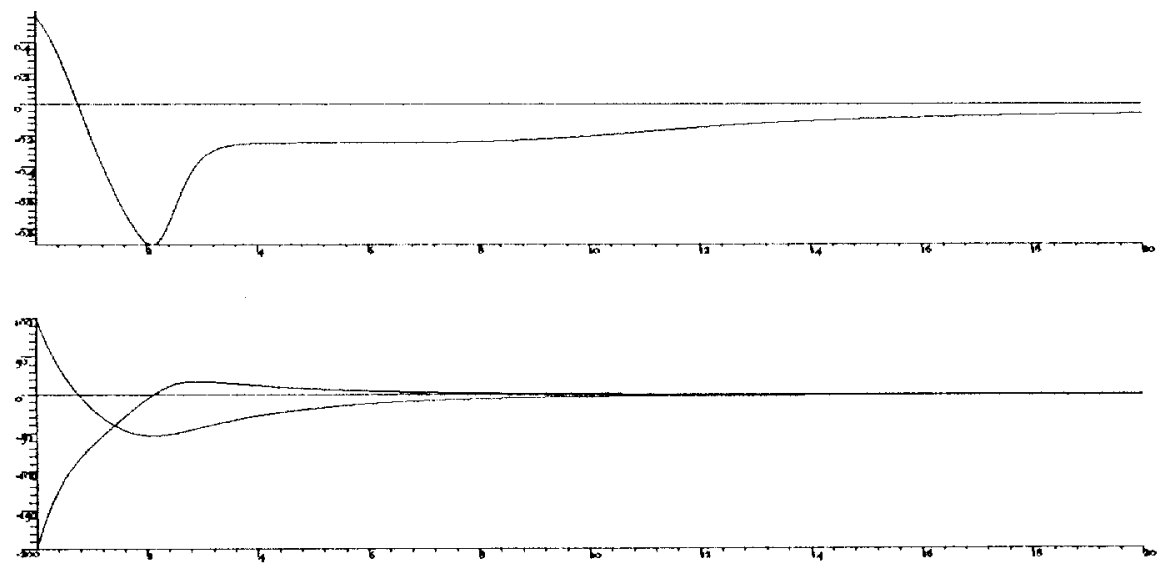

Figure 0.7: Ejemplo 6.4. Evolución de la función RCA (5.100) y abajo, los correspondientes estados, para la condición inicial $\bar{x}=(100,-200)$. 


\section{CONCLUSIONES}

En el Capítulo 2, construmos un control subóptimo con el objeto de estabilizar asintóticamente, desde una perspectiva local, cualquier sistema lineal controlable. Para este método. las ganancias $h^{\top}(x)$ se fijan en una región apropiada (vecindad) del origen con lo che se obtiene un rediseñamiento del control dado en [27]. La técnica presentada en ese capítulo se aplica al $n$-integrador, para el cual probamos que el control continuo y acotado resultante es un estabilizador asintótico y global. En la Sección 2.5, mostramos que puede hallarse una solución al problema del control continuo y acotado para los sistemas en el plano.

En el Capítulo 3, se resuelven dos problemas importantes que no pueden ser resueltos, o no se sabe muy bien cómo abordar, usando los métodos ahí mencionados: ( $i$ ) diseñar un estabilizador global continuo de manera tal que, en una vecindad del origen, el sistema a lazo cerrado tenga sus eigenvalores dentro de cierta región deseada, y (ii) diseñar un control continuo y acotado para sistemas con eigenvalores con parte real positiva. Nuestro diseño está basado en la siguiente idea geométrica. Para un sistema lineal considerado en ( $i$, existe una familia $\tau$-parametrizada de retroalimentaciones lineales saturadas $u_{\tau}(x)$ que estabilizan asintóticamente el sistema. A medida que el parámetro $\tau$ tiende al infinito, el sistema a lazo cerrado se aproxima al de lazo abierto. En particular, existen vecindades elipsoidales invariantes del origen, $\mathcal{E}(\tau)$, que tienden a ser todo $\mathbb{R}^{n}$ cuando $\tau \rightarrow \infty$, y tales que $u_{\tau}$ no se satura en $\mathcal{E}(\tau)$, para cada $\tau \in(0, \infty)$. Por consiguiente, se resuelve el siguiente problema (estabilización semiglobal): Dado un conjunto acotado $D \subset \mathbb{R}^{n}$, existe $\tau \in(0, \infty)$ tal que el control lineal saturado correspondiente $u_{\tau}(x)$ hace que $D \subset \mathcal{E}(\tau)$. Un inconveniente del enfoque semiglobal consiste en que si se tiene una región de estabilidad grande implica una dinámica lenta (ganancias pequeñas). Este problema se resuelve diseñando una función dependiente de los estados $\tau(x)$, y se propone un control de tipo lineal con ganancias dependientes de los estados que, en ese capítulo, es de la forma

$$
u(x)=-B^{\top} R(\tau(x)) x
$$

donde $R(\tau)$ es la solución de una familia $\tau$-parametrizada de (LQR) ecuaciones algebraicas de Riccati.

En el Capítulo 4 abordamos el problema de control acotado para la estabilización de los sistemas lineales. El problema de estabilización fue planteado como un problema de programación paramétrica (basado en la función de Lyapunov), donde la idea es estimar la mayor ganancia del control de una retroalimentación lineal tal que la entrada de control no exceda sus cotas. El control resultante es de tipo lineal con ganancias dependientes de los estados (i.e. $K(x) x)$. Uni característica del control propuesto radica en que la "ganancia del control" $K(x)$ se incrementa a medida que la trayectoria del sistema a lazo cerrado se aproxima al origen. Este enfoque de diseño hace un balance entre la ganancia del control $K$ con la posición $x$, de forma tal que la entrada de control resultante $u(x)=-K(x) x$ satisface las cotas prescritas. Para los sistemas lineales que son Lyapunov estables a lazo abierto, se prueba la estabilidad global asintótica. Para el caso de los sistemas lineales que son asintóticamente controlables al origen con controles acotados, el diseño se hizo en dos etapas. En la primera, se diseñó un control de baja ganancia 
Vía una ecuación algebraica de Riccati para obtener la estabilidad asintútica semiglobal. Como el diseño de baja ganancia da lugar a dinámicas "perezosas" y convergencia lenta (de hecho, para puntos cercanos al origen el control no es usado a toda su capacidad), en una segunda etapa el control de baja ganancia se rediseña para introducir un componente de alta ganancia. Tal componente de alta ganancia de la retroalimentación se construye via un problema de programación paranetrizado. Una característica central del diseño de baja-ganancia/alta-ganancia reside en que prescrua la región de atracción inducida por el diseño de baja ganancia. En ese capítulo, se incluyeron algunos ejemplos de simulación para ilustrar la implementación y desempeño de lat estralegia propuesta de control. Se hicieron comparaciones con controles lineales saturados. y se señáó que. al menos para los ejemplos estudiados, el diseño de baja-ganancia/alta-ganancia tiene un mejor desempeño que los diseños basados en saturaciones lineales.

El enfogue geométrico para la estabilización con controles acotados prescutado en ese capítulo se generaliza a ciertos sistemas afines no lineales: los sistemas pasivos. Este trabajo constituye el material del último capítulo.

Así. en el Capítulo 5 afrontamos el problema de estabilización global asintótica mediante control acotado de una clase de sistemas no lineales que son Lyapunov estables a lazo abierto. El problema de estabilización se plantea como uno de programación paranetrizada (basaco en la finción de Lyapunov). El procedimiento resultante implica que las ganancias, como funciones de lus estados, son la solucion de un programa no lineal c-parametrizado. donde se define ma sucesión de conjuntos invariantes de tamaño decreciente, asociarla con la función de Lyapunor. Relacionado con cada conjunto invariante, se elige la mayor ganancia posible, bajo la condición de que el control se mantenga acotado. Para ma clase importante de sistemas homogéneos no lineales (que a su vez, inchye una clase do sistemas que puoden ser estabilizados global asintóticamente por retroalimentaciones lineales y los sistemas bilineales), se prueba que el problena de programación resultante puede resolverse explicitamente, y adenás, también se aborda el problema de entradas sujetas a derivadas globalmente acotadas. Con referencia al caso general no lineal, tal como los sistemas bilineales no honogeneos, ol sistema a lazo cerrado resultante está definido implícitamente, en el sentido de que la ley de control se obtiene de la solución de una ecuación algebraica no lineal. En muchas aplicaciones, la restricción sobre el control se define como un $r$-hipercubo (la bola $\alpha$-normada) m-dimensional $\mathcal{B}_{r}^{\infty}=\left\{u \in \mathbb{R}^{n}\right.$ : $\left.\|u\|_{\infty}:=\max _{i}\left|u_{i}\right| \leq r\right\}$. Empero, si suponemos tal restricción, el problema de programación c-parametrizado seria no suave, y por ende sumamente difícil de resolver. Por lo tanto, se introdujo una bola de radio $r>0$, p-nomada, $\mathcal{B}_{r}^{p}=\left\{u \in \mathbb{R}^{m}:\|u\|_{p} \leq r\right\}$ a fin de obtener, al incrementar $p$, cualquer grado de aproximación suave a la restricción del control $\mathcal{B}_{r}^{\infty}$. De esta foma, se puede hacer buen uso de todo el recurso disponible (magnitud) de control.

Un aspecto objetable a esta última metodología se hace patente crando se aplica a sistenas que aunque sean asintóticamente estables a lazo abierto, sus trayectorias tengan una convergencia relativamente lenta al origen. En este caso, tenomos que si las trayectorias del sistema a lazo cerrado se aproximan a la región definida por $N=\left\{x \in \mathbb{P}^{n}: u(x)=0\right\}$-donde el control se anula. entonces se recupera la dinámica del sistema a lazo abierto, presentándose así un. pobre desempeño mediante este diseño de control. Esta desventaja es heredada del diseño del estabilizador no (necesariamente) acotado, $r(x)=-p\left(L_{y} V(x)\right)^{\top}$, cuya expresión simplemente se propone con el objeto de que la derivada de la función de Lyapmov $V(x)$ sea no positiva a lo largo de las trayectorias del sistema a lazo cemado: $d V / d t=L_{j} V(x)+L_{9} V(x) r(x)$.

Aquí podemos dar una interpretación geométrica de este fenómeno que, para sinplificar la 
discusión, la expondremos para el caso de sistemas lineales. Si nos basamos en el Capítulo 4 , la función $\tau(x)$ se define explícitamente y sus conjuntos de nivel son elipsoides concéntricos que no presentan rotaciones entre sí. Así, la región $N$ (que es un subespacio lineal) se mantiene inalterada al aplicar el control acotado. Por el contrario, si nos basamos en los diseños de los Capítulos 2 y 3 , la existencia de tales rotaciones entre los elipsoides destruye ese subespacio. redundando en un mejor desempeño. Por consiguiente, se requiere proponer otros diseños de control para el caso de sistemas no lineales que sustituyan la dinámica del sistema a lazo abierto. Un enfoque que podría resultar útil en tal dirección, se expone en [21]. En ese trabajo se proponen ecuaciones algebraicas de Riccati "congeladas" (frozen), cuyas matrices dependen de los estados. con la finalidad de obtener controles óptimos para sistemas afines. 



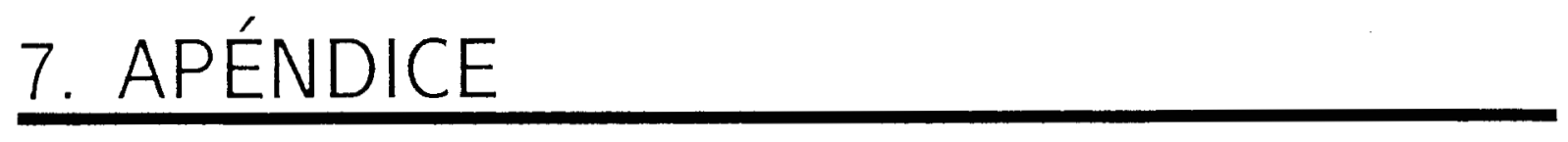

En este apéndice presentamos un breve panorama de los resultados genéricos de la programación 1-paramétrica no lineal. Con el objeto de dar una presentación sencilla del tema, hemos acotado las conclusiones más relevantes dentro del contexto de nuestro problema de optimización en control.

Consideremos una familia de programas c-parametrizados

$$
\begin{gathered}
\max _{x} f(x) \\
\text { t.q. } \quad x \in g^{-1}(c)
\end{gathered}
$$

donde $g^{-1}(c):=\left\{x \in \mathbb{R}^{n}: g(x)=c\right\}, c \in \mathbb{R}$ es el parámetro, $y f, g \in C^{k}\left(\mathbb{R}^{n}, \mathbb{R}_{+}\right), k \geq 2$. Asociemos a (7.1) el siguiente Lagrangiano

$$
L_{\lambda}(x)=f(x)-\lambda(g(x)-c)
$$

para $c>0$ fijo. El conjunto de restricciones $g^{-1}(c)$ es regular ssi para torla $x \in g^{-1}(c)$. $\nabla_{x} g(x) \neq 0$. En este caso, $c$ se llama un valor regular de $g$, denotado $g \pitchfork c$. Sea $g^{-1}(c)$ un conjunto regular, entonces un punto $x^{*} \in \mathbb{R}^{n}$ es un punto crítico (o estationario) del programa $P(c)$ ssi existe $\lambda^{*}$ en (7.2) tal que satisface $\nabla_{x} L_{\lambda^{*}}\left(x^{*}\right)=0$. El punto crítico $x^{*}$ es no degenerado ssi $\nabla_{x x} L_{\lambda^{*}}\left(x^{*}\right)$ es no singular en $\operatorname{ker} \nabla_{x} g\left(x^{*}\right)$ (i.e., en el espacio tangente de $g^{-1}(c)$ en $x^{*}$, denotado también $T_{x *} g^{-1}(c)$ ).

Entre las definiciones (o restricciones) que pueden introducirse para obtener resultados genéricos y programas "bien comportados", podemos considerar a los programas de Morse [10] y a los programas regulares [22]-[11]. En el caso del programa $P(c)$. ambos conceptos son equivalentes. Una función $f$ se denomina función de Morse en un conjunto restricción regular $g^{-1}(c)$ ssi todos los puntos críticos del programa $P(c)$ son no degenerados. Una función $f$ se dice separante ssi puntos críticos distintos tienen distintos $f$-valores. $P(c)(7.1)$ se dice que es un programa regular (separante) ssi $f$ es una función de Morse (separante) en el conjunto restricción regular $g^{-1}(c)$.

Respecto a nuestro problema de control (5.21) -Capítulo 5, la definición de un programa regular es:

(i) Todo $x \neq 0$ es un punto regular de la función de Lyapunov $V(x)(=g(x)$ en $($ T.1 $)$ ), i.e. 0 es el único mínimo de $V(x) ; y$

(ii) todos los puntos críticos $x^{*}$ de $(5.21)$, son no degenerados: $\operatorname{det}\left(\nabla_{x x}\left\|h\left(x^{*}\right)\right\|_{p}\right) \neq 0$.

En [10], se probaron los siguientes resultados, parafraseados en el presente contexto.

Teorema A.1. [10] Si $x^{*}$ es un punto crítico de un programa regular $P(c)$, entonces

(a) existe un úmico multiplicador de Lagrange $\lambda^{*} \in \mathbb{R}$ tal que $\nabla_{x} L_{\lambda^{*}}\left(x^{*}\right)=0$;

(b) la matriz hessiana de $f$ en $x^{*} \in g^{-1}(c), \nabla_{x x} L_{\lambda^{*}}\left(x^{*}\right)$, induce un isomorfismo en $T_{x^{*}} g^{-1}(c)$ : 
$\xi$

(c) Ia matriz $\nabla_{x x} L_{\lambda^{*}}\left(x^{*}\right)$ es definida negativa en $T_{x} \cdot g^{-1}(c)$ ssi $x^{*}$ es un máximo local. $\square$

Además, también se probó la siguiente caracterización de programas de Morse.

Teorema A.2. [10] El problema de optimización c-parametrizado $P(c)$ es un programa de Morse ssi $c \in C:=\left\{c \in \mathbb{R}: g \pitchfork c y\left(L_{\lambda^{*}}\left(x^{*}\right), g(x)-c\right) \pitchfork 0\right\}$.

El cerema anterior simplemente establece que $f$ es una función de Morse en $g^{-1}(c)$ ssi todos los puntos criticos de $L_{\lambda}(x)$ son no degenerados (i.e., $\left.\nabla_{x} L_{\lambda}(x) \pitchfork 0\right)$.

El programa $P(c)$ es propio ssi g es una función propia (i.e. para cualquier $c \geq 0 \cdot g^{-1}(c)$ es un conjunto compacto). En este caso, tenemos [10]: (i) $C$ es abierto, y (ii) el numero de puntos críticos dol programa $P(c)$, denotado $\# P(c)$. es finito para cualquier $c \in C y$, si $;=P(c)$ se toma como una función, es una función localmente constante en el abierto $C$.

Los programas de Morse vienen a ser interesantes para optimización ("bien comportados"). en virtud de las propiedades expuestas en los resultados anteriores. Además, bajo condiciones de suavidad de las funciones objetivo y restricción, el conjunto de programas de Morse/regulares es genérico [10]-[22]. El siguiente teorema garantiza que los programas regulares existen genéricamente (en la topología de Whitney [20]).

Teorema A.3. [11] Para c fija, el conjunto de todos los pares $(f, g)$ tales que $P(c)$ es um programa regular es $C^{2}$-abierto y $C^{2}$-denso en $C^{2}\left(R^{n}, R^{2}\right)$. El conjunto do funciones separantes de Morse en un conjunto restricción regular dado $g^{-1}(c)$ es $C^{2}$-denso y es $C^{2}$-abierto si $g^{-1}(c)$ es compreto.

La suavidad de la función de valor óptimo también es genérica [10|. Esta propiedad importante, parafrascada en el presente contexto, está dada por el siguiente resultarlo.

Teorema A.4. [10][11] Supongamos que $f(x)=v(x)+w^{\top} x$, donde $v: \mathbb{R}^{\prime \prime} \rightarrow \mathbb{R}$ es una función $C^{k}(k \geq 2)$ y $w \in \mathbb{R}^{n}$, y también que $g \in C^{n}$, donde $n$ es la dimensión del espacio, $y$ propia. Entonces, para casi todo par $(c, w) \in \mathbb{R}_{+} \times \mathbb{R}^{\prime \prime}$, el problema de optimizición (7.1) es un programa de Morse que tiene a lo más una solución global. Además, la solución global $a^{*}(c . w)$ de (T.1) es $C^{1}$ y su máximo valor global $f(c, w)$ es $C^{2}$ sobre un conjunto abierto y denso en el espacio de perturbación de parámetros.

Respecto al contexto de control escalar dado en la Sección 4.1 del Capítulo 5, el teorema anterior establece que si el control escalar contiene un término lineal ( $\left.w^{\top} x\right)$, entonces el programa resultante (5.39) es "bien comportado", en los sentidos presentados antes.

Por otra parte, en [22], se estudió el conjunto de puntos criticos de un programa unoparamétrico, y en particular, cl cambio del indice de Morso $(\mathrm{m})$ [42] con el parámetro. El índice de Horse $m$ en $x^{*}$ denota el mimero de eigenvalores negativos de la matriz hessiana de $f$ en $x^{*} \in g^{-1}(c)$ (i.e., $\nabla_{x x} L_{\lambda^{*}}\left(x^{*}\right)$ ), restringido al espacio tangente de $g^{-1}(c)$ en $x^{*}\left(T_{x} g^{-1}(c)\right)$. Así. el indice $\mathrm{m}$ determina la naturaleza de un punto crítico $x^{*}$ : (a) si $\mathrm{m}=n$, entonces $x^{*}$ es una solución máxima local; (b) si $\mathrm{m}=0$, entonces $x^{*}$ es una solución minima local; mientras que (c) si $1 \leq \mathrm{m} \leq n-1$, entonces $x^{*}$ es una solución del tipo pruto silla.

Teorema A.5. [22] Sea $\emptyset \neq \Sigma$ el conjunto de puntos críticos de un programa regular parametrizado (7.1). Entonces $\Sigma$ es una $C^{2}$-variedad 1-dimensional de $\mathbb{R}^{n+1}$. Además, el indice de Morse $m$ es constante en cadia componente de $\Sigma_{1} \subset \Sigma$. donde $\Sigma_{1}$ denota d conjunto de puntos críticos no degenerados. 
Resumiendo, en el enfoque de Jongen et al. [22], se probó que genéricamente el conjunto de puntos críticos constituye una unión ajena de curvas seccionalmente suaves en $\mathbb{R}^{n+1}$, y también se especifan las maneras en que las propiedades de segundo orden (en términos de $\mathrm{m}$ ) de los puntos críticos pueden cambiar conforme varía el parámetro.

Se puede conseguir en la literatura, cf. por ejemplo [16]-[41], métodos para el seguimiento de trayectorias (pathfollowing), la determinación del tipo de solución, y la detección de singularidades en los problemas de programación paramétrica no lineal. 



\section{Bibliografía}

[1] .J. Ackermann, Robust Control: Systems with Cincertain Physical Parameters. SpringerVerlag, London, C.K.., (1994).

[2] J. Alwarez-Ramírez, R. Sucrez and J. Álvarez, Semi-global stabilization of multi-imput linear systems with saturated linear state feedback, Syst. E. Control Lett.. 23 (1994), 247-254.

(3) A. Andreini, A. Bacciotti, P. Boieri and G. Stefani, Stabilization of nonlinear systems by means of linear feedbacks, in Proc. Int. Conf. Nonlinear Systems, Lect. Notes Control Inform. Sci. 122, Springer-Verlag, N.Y., (1988), 281-289.

[4] (․ Burgat, S. Tarbouriech and M. Kai, Continuous-time saturated state feedback regulators: Theory and design, Int. J. Syst. Sci, 25 (1994), 315-336.

[D] C.I. Bymes. A. Isidori and J.C. Willens, Passivity, feedback equivalence, and the global stabilization of mininum phase nonlinear systems, IEEE Trans. Autom. Control. 36 (1991). $1228-1240$

[6] M. Corless and G. Leitman, Exponential convergence for uncertain systems with component-wise bounded controllers, in Robust Controb via Variable Structure and Lyapunov Techniques, F. Garofalo and L. Glielmo, eds., Lect. Notes Control Inform. Sci. 217. Springer-Verlag, London, (1996), 175-196.

[i] F.L. Chernous'ko, Ellipsoidal estimates of a controlled system's attainability domain, .J. Appl. Math. Wechs, 45 (1982), T-12.

[8] J.F. Frankena and R. Sivan, A non-linear optimal control law for linear systens, Int. J. Control, 30 (1979), 159-178.

[9] G.F. Franklin, E.D. Powell and A. Emami-Naeini, Feedoack Control of Dynamic System.s, Addison-Wesley, Reading, MA, (1991).

[10] O. Fujiwara, .Morse programs: A topological approach to smooth constrained optimization. I, Math. Oper. Res., 7 (1982), 602-616.

[11) O. Fujwara. A note on differentiability of global optimal values, Math. Oper. Res., 10 $(1985), 612-618$.

[12] A.T. Fuller. In-the-large stability of relay and saturated control systems with linear controllers, Int. J. Control, 10 (1969), 457-480. 
[13] F. Canmaher. The Theory of Matrices, Vol. 1, Chelsea, N.Y., (1959).

[14] J. P. Gumer and I. Fupka, A separation principle for bilinear systems with dissipative dritt. IEEE Trans. Autom. Control. 37 (1992). 1970-1974.

[15] V.M. Gamilyako, V.I. Korobov and G.M. Sklyar, Designing a bounded control for dynamie sisterns in entire space with the aid of a controllability function, Autom. Remote Control. $11: 1986)$. : $184-1490$.

[16] .I Guldat. F. Guerra Vézquez and H. Th. Jongen. Purametric Optimization: Singularities. I'athfolloung and Jumps. Teubner and J. Wiley \& Sons, Chichester, GB, (1990).

[17, P.-O. Guman, Stabilizing controllers for bilinear systems. IEEE Truns. Autom. Control. 26 (1981), $917-922$.

[18] O. Háyek. Geometric theory of time-optimal control, SIAM J. Control, 9 (1971), 339-350.

(19] 1). Hill and P. Moylan, The stability of nonlinear dissipative systems, IEEE Trans. Autom. Control, 21 (1976), 708-711.

[20] M. W. Hirsch, Differential Topology, Springer-Verlag, X.Y., (1976).

21] Y. Huang and W.-M. Lu, Nonlinear optimal control: Alternalives to Hamilton-Jacobi equation, in Proc. 35th. IEEE Conf. Dec. Control, Kobe, Japan, (Dec. 1996). 3912-3947.

[22] H. Th. Jongen, P. Jonker and F. Twilt, One-parameter families of optimization problems: Equality constraints, J. Optim. Theory Appl.. 48 (1986), 141-161.

[23] レ. Jurdjeric and J. P. Quinn, Controllability and stability, I. Diff. Equations., 28 (1978), $381-389$.

[24] $\therefore$. Kalouptsidis and J. Tsinias, Stability improvement of nonlinear systems by feedback, IEEE Trans. Autom. Control, 29 (1984), 364-367.

[25] N. Kawasaki and E. Shimemura, Determining quadratic weighting matrices to locate poles in a specified region. Automatica, 19 (1983), 557-560".

[26] V.A. Tomarov, Estimates of reachable sets for linear systems, Math. USSR Izvestiya, 25 (1985), $193-206$.

[27] V.A. Komarov, Design of constrained controls for nonautonomons linear systems, Autom. Remote Control, 10 (1985), 535-539.

[28] V.I. Korobov, A general approach to the solntion of bounded control syuthesis problem in a controllability problem, Math. USSR Sbornil, 37 (1980), 535-5.57.

[29] V.I. Korobor and G.M. Sklyar, Time optimality and the power monent problem, Malh. USSR Shomin, 62 (1989), 185-206.

[30] V.I. Korobov and G.M. Sklyar, Time-optimal control and the trigonometric moment problem, Math. USSR Izlestiya, 35 (1990), 203-220. 
[31] K. K. Lee and A. Arapostathis. Remarks on smooth feedback stabilization of nonlinear systems. Syst. \& Control Lett., 10 (1988), 41-14.

[32] E.B. Lee and L. Markus, Foundations of Optimal Control Theory. J. Wiley \& Sons, X.Y.. $(1967)$

[33] Y. Lin and E. D. Sontag. A universal formula for stabilization with bounded controls, Syst. G Control Lett., 16 (1991), 393-397.

[34] W. Lin. Input saturation and global stabilization by output feedback for affine systems, in Fror. 33 d. IEEE Conf. Dec. Control. Lalie Buena Vistic, FL. (Dec. 1994), 1323-1328.

[35] IV. Lin. Global asymptotic stabilization of general nonlinear systems with stable free dynamics via passivity and bounded feedback. 4utomatica, 32 (1996), 915-924.

[36] Z. Lin. Semi-global stabilization of linear systems with position and rate limited actuators, Syst. Control Lett., 30 (1997), 1-11.

[37] Z. Lin and A. Saberi, Semi-global exponential stabilization of linear systems subject to "input saturation" via linear feedbacks, Syst. \& Control Lett., 21 (1993), 225-239.

[38] Z. Lin and A. Saberi, Semi-global stabilization of minimum phase nonlinear systems in special normal form via linear high-low-gain state feedback, Int. J. Robust Nonlinear Control. 4 (1996), 353-362.

[39] R. Longchamp, State-feedback control of bilinear systems, IEEE Trans. Autom. Control, $25(1980), 302-306$.

[40] D.G. Luenberger, Linear and Nonlinear Programming, 2nd. Ed., Addison-Wesley, Reading. MA, (1984).

[-11] B. ․ Lundberg and A. Poore, Numerical continuation and singularity detection methods for parametric nonlinear programming, SIAM J. Optim., 3 (1993), 134-154.

[42] J. Milnor, Morse Theory, Annals . Cath. Stud. 51, Princeton Univ. Press, NJ, (1.963).

[43] R. Mohler, Billinear Control Processes, Academic Press, N.Y., (1973).

[44] B.P. Molinari, The time-invariant linear-quadratic optimal control problem, Automatica. $13(197 \vec{\imath}), 347-357$.

[45] M. Morari and E. Zafiriou, Robust Process Control, Prentice-Hall, Englewood Cliffs, N.J, (1989).

[46] A.I. Ovssevich and F.L. Chemousko. Two-sided estimates on the attainability domains of controlled systems, J. Appl. Math. Mech.s. 46 (1983), 590-595.

[47] M.A. Ponbelle, R.R. Bitmead and M.R. Gevers, Fake algebraic Riccati techniques and stability, IEEE Truns. Autom. Control, 33 (1988), 379-381.

[48] A. Saberi, Z. Lin and A.R. Teel, Control of linear systens with saturating acturtors, IEEE Trans. Autom. Control, 41 (1996), 368-378. 
[-49] W.E. Schmitendorf and B.R. Barmish, Null controllability of linear systems with constrained controls. SHA.K J. Control Optim. 18 (1980), 327-345.

[50] M. Stenmod. Stabilization of bilinear control systems with applications to nonconservative problems in elasticity. SLAMJ. Control Optim. 16 (1978), 131-111.

[51] L.S. Shieh. H.M. Dib and B.C. Mcinnis. Linear quadratic regulators with eigenalue placement in a vertical strip. IEEE Trans. Autom. Control, 31 (1986), 241-243.

152] J. Sulís-Daun, Estimación de conjuntos alcanzables de sistemas linealizables. Tesis de Mastria, Entersidad Autónoma Metropolitana-Iztapalupu. (Oct. 1989).

[53] J. Solís-Daun, J. Álvarez and R. Suárez, Bonnded finite time control for planar feedback linearizable systems: Application to a continuous stirred tank reactor, in Proc. Amer. Control Conf., Chicago IL, (June 1992), 1018-1022.

[54] J. Solís-Dann. J. Álvarez-Ramírez and R. Suárez, Global and semi-global stabilization of linear systems using constrained controls, (1997), sometido al Int. .J. Robust Nonlinear Control.

[55] J. Solis-Dam, R. Surrez and J. Álvarez-Ramírez. Global stabilization of nonlinear systems using bounded control: A parametric optimization approach. (1997). sometido al SIAM I. Control Optim.

56| E.D. Sontag, An algebraic approach to bounded controllability of linear systens, Int. I. Control, $39(1984), 181-188$.

[ji] E.D. Sontag and H.J. Susmann, Nonlinear ontput feedback design for linear systems with saturating controls, in Proc. 29th IEEE Conf. Dec. Control, Honolulu [II, (Dec. 1990), $3414-3416$.

[58] R. Sućrez, J. Alvarez and J. Álvarez, Linear systems with single sutuated input: Stability analysis, in Proc. 30th. 1EE Conf. Dec. Control, Brighton, England, (Dec. 1991), 223-228.

[59] R. Suárez, J. Alvarez-Ramírez and J. Alvarez, Regions of attraction of closed loop linear systens with satumated linear leedback, J. Math. Syst. Estim. Control, (1997), tru prensa.

[60] R. Suárez, J. Álvarez-Ramirez and J. Solís-Danu, Linear systcus with bonnded inpuls: Global stabilization with eigenvahe placement. Int. J. Robust Nonlinear Control. $7(1997)$. $835-845$.

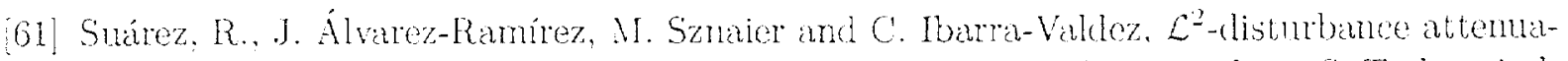
tion for linear systems with bounded controls: An ARE-based appronch, in S. Tarbouriech and G. Garcia (Els.). Control of Uncertain Systems with Boundell Inputs, Lect. Notes Control Inform. Sci. No. 227. Springer-Veriag. Londun. U.K., (1997), 25-38.

[02] R. Suárez, J. Solis-Dann and J. Alvarez, Stabilization of lincur controllable systens by means of bounded continuons nonlinear leedback control, Syst. \& Control Lolt. 23 (1994). $403-410$. 
[63] H.J. Sussmann and Y. Yang, On the stability of multiple integrators by means of bounded feedback controls, in Proc. 30th IEEE Conf. Dec. Control, Brighton. England, (Dec. 1991). $70-i 2$.

[64] H.J. Sussmann, E. Sontag and Y. Yang, A general result on the stabilization of linear systems using bounded controls, IEEE Trans. Autom. Control, 39 (1994), 2411-2424.

[65] A.R. Teel, Global stabilization and restricted tracking for multiple integrators with bounded controls, Syst. \& Control Lett., 18 (1992), 165-171.

[66] A.R. Teel, Feedback stabilization: nonlinear solutions to inherently nonlinear problems, Memo No. UCB/ERL MO2/65, University of California, Berkeley CA, (June 1992).

[67] A.R. Teel, Linear systems with bounded nonlinearities: Global stabilization by scheduling a family of $H_{x}$-type controllers, Int. J. Robust Nonlinear Control. 5 (1995), 399-411.

[68] J. Tsinias, A theorem on global stabilization of nonlinear systems by linear feedback, Sy.stem.s Control Lett., 17 (1991), 357-362.

[69] W.M. Whonam, Linear Multivariable Control: A Geometric Approach, Springer-Verlag, N.Y., (1985).

[70] J. C. Willems, Dissipative dynamic systems-Part I: General Theory; -Part II: Linear systems with quadratic supply rates, Arch. Rational Mechs. Analysis, 45 (1972), 321-351 and 352393.

171) G.F. Wredenhagen and P.R. Bélanger, Piecewise-linear LQ control for systems with input constraints, Automatica, 30 (1994), 403-416.

[72] Y. Yang, H.J. Sussmann and E.D. Sontag, Stabilization of linear systems with bounded control, in Proc. IFAC Conf. Nonlinear Control Systems Design, Bordeaux, France, (June 1.992), 15-20.

[73] D.S. Yeung, Time-optimal feedback control, J. Optim. Theory Appl., 21 (1977), 71-82.

[74] Q. Zhou, A transversality theorem for maps, J. Math. Res. Exposition, 3 (1983), 17-20, (en chino, con resumen en inglés). 